Aus der Klinik für Unfallchirurgie, Orthopädie und Plastische Chirurgie

(Prof. Dr. med. W. Lehmann)

der Medizinischen Fakultät der Universität Göttingen

\title{
Langzeitergebnisse von operativ versorgten Wirbelsäulendeformitäten bei Kindern mit Spinaler Muskelatrophie
}

\author{
INAUGURAL-DISSERTATION \\ zur Erlangung des Doktorgrades \\ der Medizinischen Fakultät der \\ Georg-August-Universität zu Göttingen
}

vorgelegt von

Marina Magdalena Hecker

aus

Saarbrücken

Göttingen 2020 
Dekan:

\section{Betreuungsausschuss}

Betreuer/in

Ko-Betreuer/in:

\section{Prüfungskommission}

Referent/in

Ko-Referent/in:

Drittreferent/in:
Prof. Dr. W. Brück

Prof. Dr. med. A. K. Hell

Prof. Dr. med. H.-C. Ludwig

Datum der mündlichen Prüfung: 
Hiermit erkläre ich, die Dissertation mit dem Titel "Langzeitergebnisse von operativ versorgten Wirbelsäulendeformitäten bei Kindern mit Spinaler Muskelatrophie" eigenständig angefertigt und keine anderen als die von mir angegebenen Quellen und Hilfsmittel verwendet zu haben.

Göttingen, den

(Unterschrift) 
Die Daten, auf denen die vorliegende Arbeit basiert, wurden teilweise publiziert:

Lorenz HM, Badwan B, Hecker MM, Tsaknakis K, Groenefeld K, Braunschweig L, Hell AK (2017): Magnetically controlled devices parallel to the spine in children with spinal muscular atrophy. JB JS Open Access 2 , e0036

Lorenz HM, Braunschweig L, Schiele S, Tsaknakis K, Hecker MM, Gantner A, Hell AK (2018): Surgical treatment of spinal deformities in young paraplegic children with intraspinal tumors. Pediatr Neurosurg $\underline{53}, 149-152$

Lorenz HM, Braunschweig L, Badwan B, Groenefeld K, Hecker MM, Tsaknakis K, Grote J, Hell AK (2019): High correlation between achieved and expected distraction using magnetic controlled growth rods with rib to pelvis fixation in pediatric spine deformity. J Pediatr Orthop $\underline{39}$, e334-e338

Hell AK, Braunschweig L, Tsaknakis K, von Deimling U, Lüders KA, Hecker M, Lorenz HM (2020): Children with spinal muscular atrophy with prior growth-friendly spinal implants have better results after definite spinal fusion in comparison to untreated patients. Neurosurgery, (im Druck) 


\section{Inhaltsverzeichnis}

Abbildungsverzeichnis ........................................................................................ VII

Tabellenverzeichnis .........................................................................................III

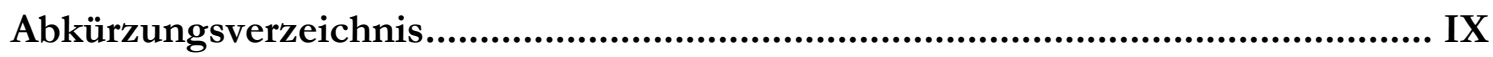

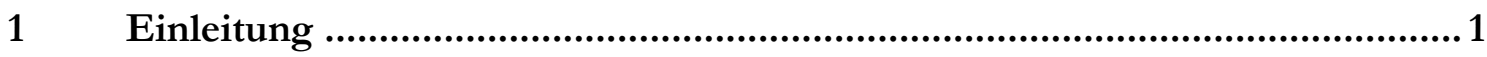

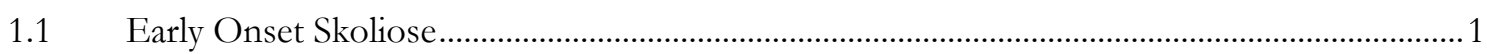

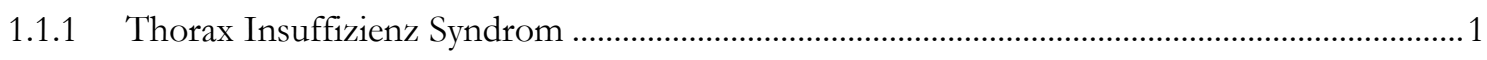

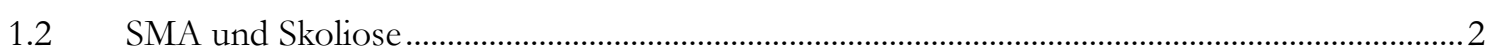

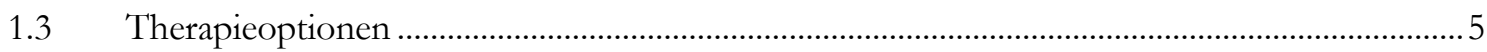

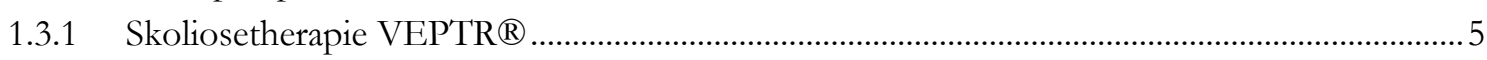

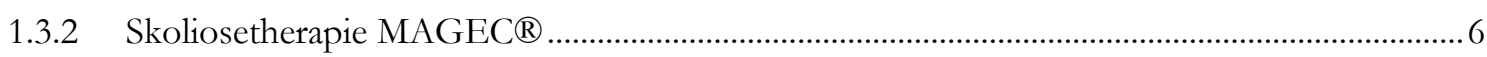

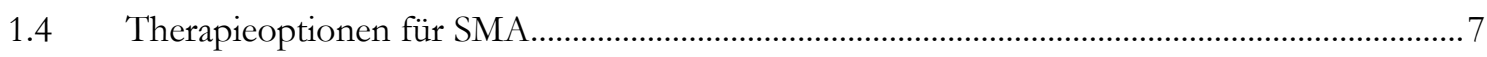

1.4.1 Symptomatische Therapie der Skoliose bei SMA ……………………......................................

1.4.2 Kausale Therapie der SMA mit Nusinersen $\left(\right.$ Spinraza $\left.{ }^{\circledR}\right)$........................................................ 8

$1.5 \quad$ Aktueller Wissensstand in der Forschung ...........................................................................

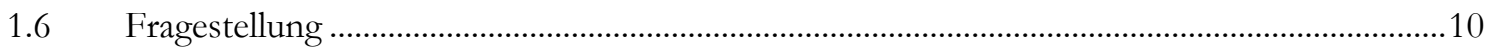

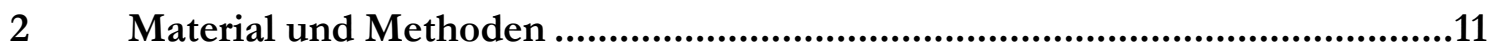

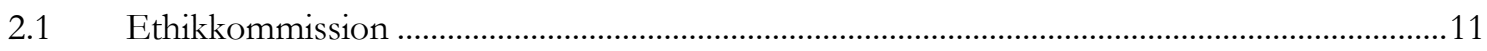

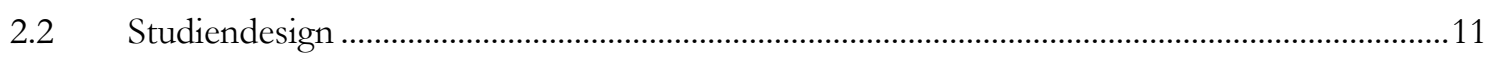

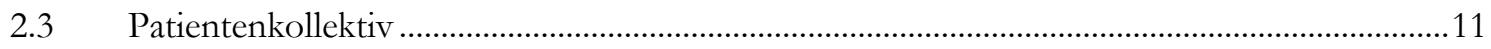

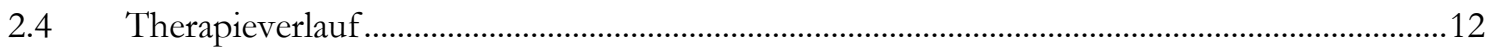

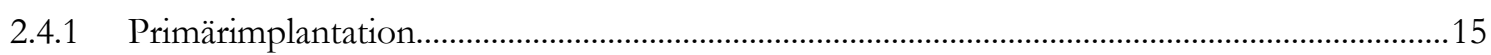

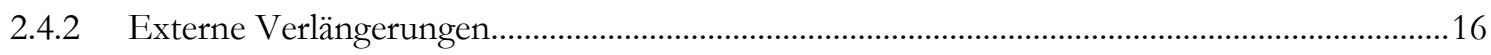

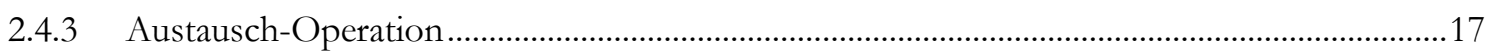

2.4.4 Externe Verlängerungen des Austauschimplantates...............................................................17

2.4.5 Spondylodese, Endpunkt der Studie ...................................................................................... 17

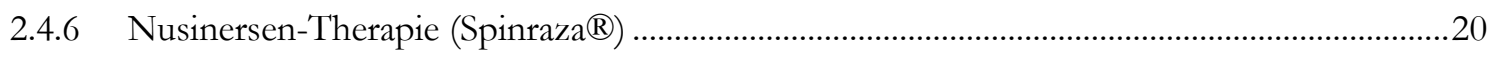

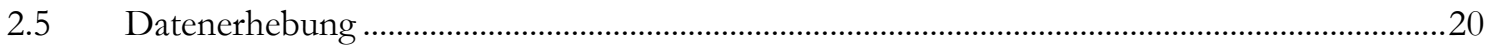

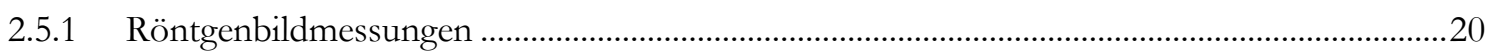

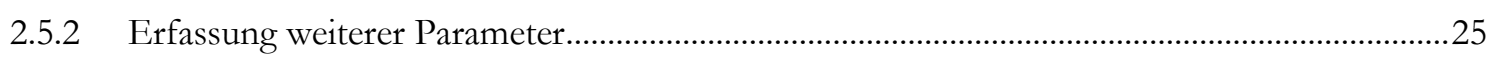

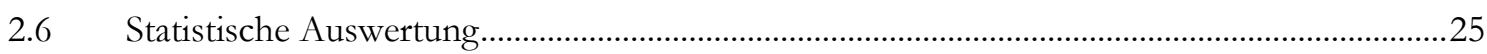

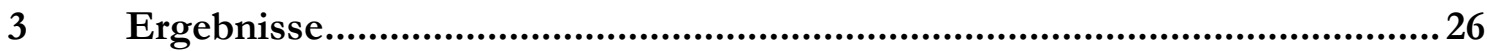

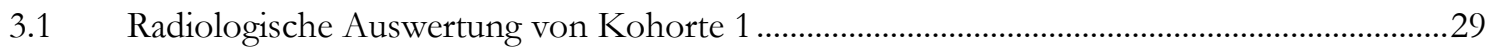

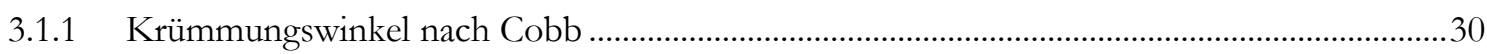

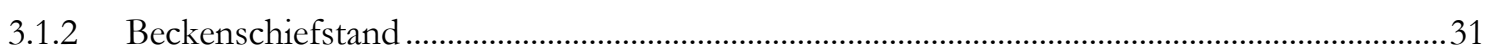

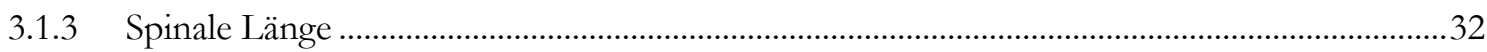

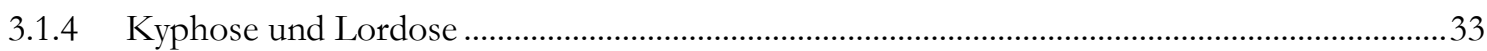




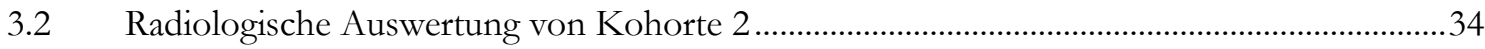

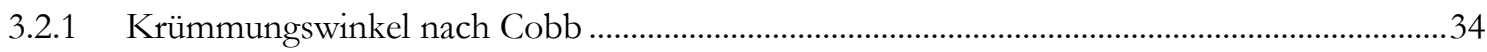

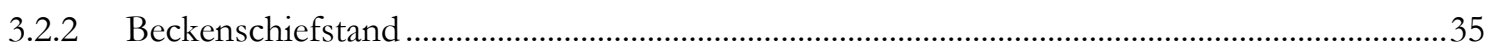

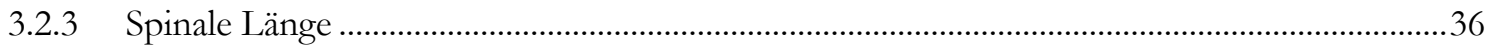

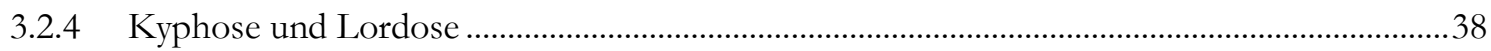

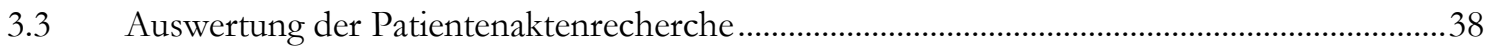

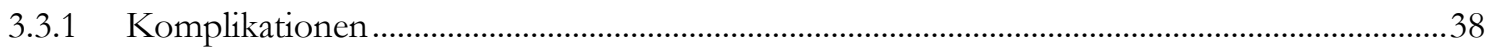

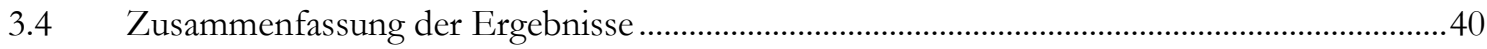

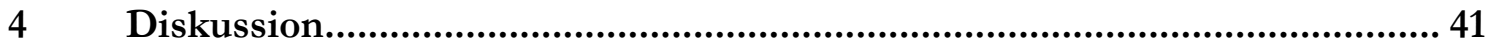

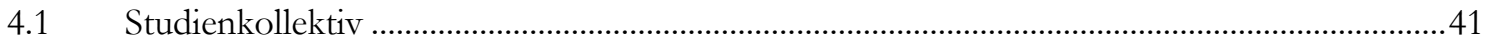

4.2 Veränderungen der radiologischen Parameter im Langzeitverlauf ........................................43

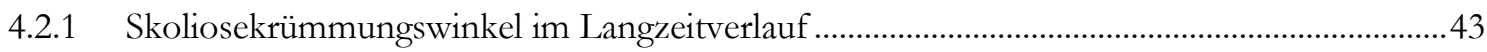

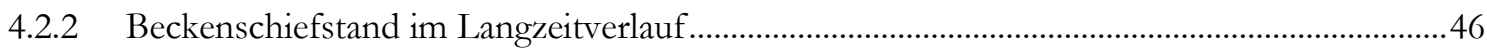

4.2.3 Spinale Länge, Kyphose- und Lordosewinkel im Langzeitverlauf .......................................47

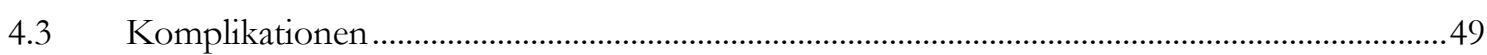

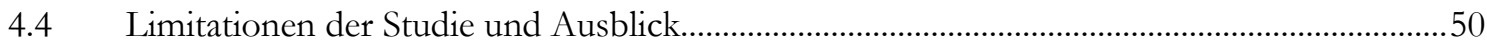

$5 \quad$ Zusammenfassung..............................................................................52

6 Literaturverzeichnis ................................................................ 54 


\section{Abbildungsverzeichnis}

Abbildung 1: Röntgenbilder eines SMA-Patienten im a.p. und seitlichen Strahlengang. ........................4

Abbildung 2: Röntgenbilder eines SMA-Patienten im a.p. Strahlengang. ................................................

Abbildung 3: Skizze des Therapieverlaufes der Kohorte 1. ...............................................................13

Abbildung 4: Skizze des Therapieverlaufes der Therapie der Kohorte 2.............................................14

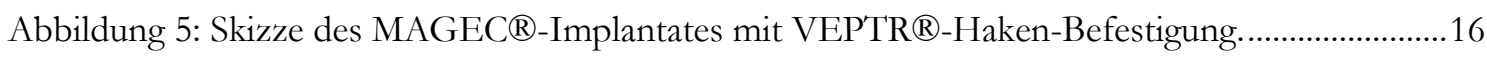

Abbildung 6: Skizze der versteiften Wirbelsäule nach der Spondylodese mit eingebrachten

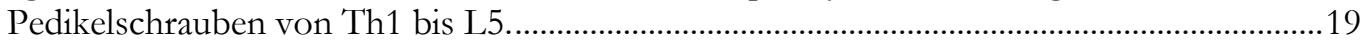

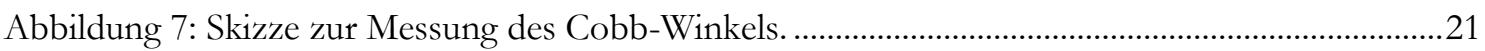

Abbildung 8: Skizze zur Messung des Beckenschiefstandes. ..................................................................22

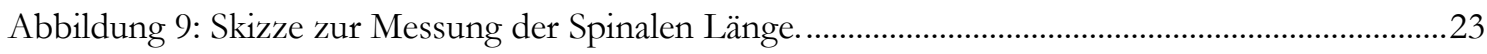

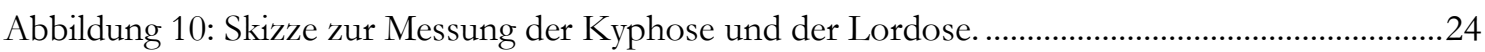

Abbildung 11: Dargestellt ist der Therapieverlauf der Kohorten 1 und 2b von Beginn bis Endpunkt der Beobachtungen am Beispiel der Röntgenbilder eines Patienten des Kollektivs.

Abbildung 12: Dargestellt sind Röntgenbilder eines Patienten der Kohorte 2a.

Abbildung 13: Ergebnisse der Messungen des Krümmungswinkels nach Cobb in der Kohorte 1 ( $\mathrm{n}=17$ Patienten) im Zeitverlauf der Verlängerungen. ............................................................30

Abbildung 14: Ergebnisse der Messungen des Beckenschiefstandes in der Kohorte 1 im Zeitverlauf der Verlängerungen.

Abbildung 15: Darstellung der Spinalen Länge der Kohorte 1 ( $\mathrm{n}=17$ Patienten) im Zeitverlauf.......32

Abbildung 16: Kyphose- und Lordosewinkel der Kohorte 1 ( $\mathrm{n}=17$ Patienten) im Zeitverlauf..........33

Abbildung 17: Darstellung des Skoliosekrümmungswinkels in Grad von der Kohorte 2b $(\mathrm{n}=14)$, das heißt von SMA-Kindern mit einer MAGEC®- oder VEPTR®-

Behandlung für durchschnittlich 4,9 Jahre vor Spondylodese (grün) im Vergleich zu der Kohorte 2a ( $\mathrm{n}=14)$ (rot), die nicht operativ vorbehandelt war.

Abbildung 18: Darstellung des Beckenschiefstandes in Grad von der Kohorte 2b ( $\mathrm{n}=14)$, das heißt von SMA-Kindern mit einer MAGEC ${ }^{\circledR}$ - oder VEPTR ${ }^{\circledR}$-Behandlung für durchschnittlich 4,9 Jahre vor Spondylodese (grün) im Vergleich zu der Kohorte 2a $(\mathrm{n}=14)$ (rot), die nicht operative vorbehandelt war.

Abbildung 19: Darstellung der Spinalen Länge in Millimetern von der Kohorte 2b ( $\mathrm{n}=14)$, das heißt von SMA-Kindern mit einer MAGEC®- oder VEPTR ${ }^{\circledR}$-Behandlung für durchschnittlich 4,9 Jahre vor Spondylodese (grün) im Vergleich zu der Kohorte 2a $(\mathrm{n}=14)$ (rot), die keinerlei operative Vorbehandlung hatten. 


\section{Tabellenverzeichnis}

Tabelle 1: Übersicht über die Charakteristika der Kohorte $1(\mathrm{n}=17)$.................................................26

Tabelle 2: Übersicht über die Charakteristika der Kohorte $2(\mathrm{n}=28)$...............................................28

Tabelle 3: Übersicht über die Anzahl und die Art der implantatspezifischen Komplikationen .........39 


\section{Abkürzungsverzeichnis}

\begin{tabular}{|c|c|}
\hline a.p. & anterior-posterior \\
\hline BMI & Body-Mass-Index \\
\hline BWS & Brustwirbelsäule \\
\hline CGR & conventional growing rod \\
\hline $\mathrm{CT}$ & Computertomografie \\
\hline EMA & Europäische Arzneimittelagentur \\
\hline EOS & Early Onset Scoliosis \\
\hline FU & Follow-ир \\
\hline $\mathrm{L}$ & Lendenwirbelkörper \\
\hline LWS & Lendenwirbelsäule \\
\hline MAGEC® & Magnetic Expansion Control \\
\hline MCGR & magnetically controlled growing rod \\
\hline MRT & Magnetresonanztomografie \\
\hline $\mathrm{N}$ & Neutralwirbel \\
\hline S & Scheitelwirbel \\
\hline SMA & Spinale Muskelatrophie \\
\hline SMN-Gen & Survival-motor-neuron-Gen \\
\hline SMN-Kopie & Survival-motor-neuron-Kopie \\
\hline SMN-Protein & Survival-motor-neuron-Protein \\
\hline TGR & traditional growing rod \\
\hline $\mathrm{Th}$ & Brustwirbelkörper \\
\hline TIS & Thorax Insuffizienz Syndrom \\
\hline UMG & Universitätsmedizin Göttingen \\
\hline VEPTR $®$ & vertical expandable prosthetic titanium \\
\hline
\end{tabular}




\section{$1 \quad$ Einleitung}

\subsection{Early Onset Skoliose}

Eine Skoliose ist per Definition eine dreidimensionale Wirbelsäulendeformität mit einer Verdrehung der Wirbelsäule und einer Krümmung in der Frontalebene von mindestens zehn Grad (Trobisch et al. 2010). Skoliotische Wirbelsäulendeformitäten werden nach unterschiedlichen Kriterien klassifiziert. Unter Beachtung der zeitlichen Erstmanifestation und der Prognose kann zwischen early und late onset Skoliosen unterschieden werden. Eine Early Onset Scoliosis (EOS) tritt schon vor dem zehnten Lebensjahr auf (Yang et al. 2016; Ridderbusch et al. 2018) und ist mit einer schlechteren Prognose behaftet als die klassische idiopathische Form. Anders als die klassische idiopathische Skoliose, ist die EOS selten idiopathisch $(<1 \%)$. Viel häufiger ist sie Syndrom-assoziiert, kongenital oder neuromuskulär bedingt. Für die Auswahl des Therapieverfahrens und für die Prognose ist unter anderem entscheidend, wie gut sich die Krümmungen passiv redressieren lassen, sprich ob die Skoliose „weich“ oder „steif“ ist. Während die strukturellen Missbildungen der kongenitalen Skoliose zu vergleichsweise steifen Deformitäten führen, sind die neuromuskulär bedingten Krümmungen im Falle muskulärer Hypotonie eher weiche Skoliosen. Grundsätzlich führen muskuläre Dysbalancen im Bereich der autochthonen Rückenmuskulatur häufig zu schweren, C-förmigen und großbogigen Skoliosen, da das koordinierte Zusammenspiel der Rückenmuskulatur einen Hauptstabilisator der Wirbelsäule darstellt. So können sich Kinder, die an hypotonen Muskeltonusstörungen erkrankt sind, nicht aufrecht halten und sacken schlaff in sich zusammen (Gillingham et al. 2006; Yang et al. 2016; Ridderbusch et al. 2018).

Bei Patienten mit einer Spinalen Muskelatrophie (SMA) bilden sich oft in jungen Jahren progrediente, zu Beginn gut redressierbare, großbogige EOS aus (Fujak et al. 2013; Yang et al. 2016).

\subsubsection{Thorax Insuffizienz Syndrom}

Die Lungenfunktion ist bei SMA-Patienten überwiegend durch ein stark verringertes Thoraxvolumen und durch eine verminderte Atemmechanik, welche erstens aus der Muskelschwäche, zweitens aus einer verminderten Beweglichkeit der Rippengelenke und drittens aus einem Zwerchfellhochstand resultiert, beeinträchtigt. Das Thoraxvolumen ist bereits in jungen Jahren oft so stark reduziert, dass die Anzahl der Alveolen und das Gesamtvolumen des Lungenparenchyms sich nicht altersentsprechend entwickeln können (Yang et al. 2016). Durch die eingeschränkte Beweglichkeit des Zwerchfells (Zwerchfellmotiliät) und vermehrte Steifigkeit des Thorax (Thoraxrigidität) kann das 
Lungenparenchym nur in einem reduzierten Ausmaß ventiliert werden. Das Vollbild der daraus resultierenden erheblichen Lungenfunktionsstörung bezeichnet man unabhängig von der Ätiologie der Skoliose als thoracic insufficiency syndrome (TIS) (Campbell et al. 2004).

Das TIS wird von Campbell beschrieben als „Unfähigkeit des Thorax beziehungsweise der Thoraxwand, eine normale Lungenfunktion und eine normale Lungenentwicklung bei Kindern im Wachstum zu unterstützen“ (Campbell et al. 2004). Klinisch bedeutet ein TIS eine restriktive Lungenerkrankung mit verminderten thorakalen Volumen, verminderter thorakalen Dehnbarkeit (Compliance), eingeschränkter Muskelfunktion und pulmonaler Hypoplasie (Watts 2016).

Im weiteren Verlauf kann durch die restriktive Lungenerkrankung eine Überbelastung des Herzens mit Herzmuskelhypertrophie (Cor pulmonale) und ein zu hoher Druck im Lungenkreislauf (pulmonale Hypertension) entstehen. Präventiv eignet sich eine Therapie der Wirbelsäulendeformität, welche auch die Thoraxdeformität mitbehandelt und sich auf möglichst physiologischer Weise dem kindlichen Wachstum anpassen lässt. Bei Betrachtung der Lungenentwicklung lässt sich feststellen, dass bereits mit dem zweiten Lebensjahr ein Großteil der Alveolen angelegt ist (Yang et al. 2016). Mit zunehmendem Alter wird die Alveolenanzahl nicht erheblich weiter ansteigen. Allerdings findet zwischen der Geburt und dem zehnten Lebensjahr die maximale Zunahme des Lungenvolumens statt (Yang et al. 2016), welche man mit dem Therapieziel, Platz im Thorax zu schaffen, unterstützt. Mehr Platz im Thorax für die Lungenentwicklung bedeutet daher weniger Beeinträchtigung der Lungenfunktion, und so lässt sich ein TIS vorbeugen (Campbell et al. 2004; Campbell und Smith 2007; Yang et al. 2016).

\subsection{SMA und Skoliose}

Die Spinale Muskelatrophie ist eine hereditäre Erkrankung des zweiten Motorneurons im Vorderhorn des Rückenmarks, die autosomal rezessiv vererbt wird und mit einer Inzidenz von 1 von 6.000 - 10.000 Säuglingen auftritt (Kolb und Kissel 2011). In dem Jahr 1990 wurde die ursächliche Deletion im 5q13 survival motor neuron (SMN)1-Gen entdeckt (Brzustowicz et al. 1990), welche zur Proteinsynthese des fehlerhaften SMN-Protein führt, das ursächlich für die Erkrankung ist (Lefebvre et al. 1995; Russman 2007). Aus der unterschiedlichen Ausprägung des Phänotyps der Erkrankung, dazu gehören Symptome wie Hypotonie, Hyporeflexie und zunehmende generelle Muskelschwäche, sowie dem Krankheitsbeginn, ergibt sich die überwiegend klinische Einteilung in die SMA-Typen I bis IV (Russman 2007; Fujak et al. 2012; Fujak et al. 2013). Bei Kindern mit SMA-Typ I ist der Krankheitsbeginn innerhalb der ersten sechs Monate nach Geburt zu erwarten. Die Kinder erlangen im Allgemeinen die Sitzfähigkeit nicht. Der Krankheitsbeginn der SMA-Typ II ist vor dem 18. Lebensmonat. Die Kinder können zwar selbstständiges Sitzen erlernen, werden aber nie selbstständig stehen können. Patienten mit SMA-Typ III erkranken nach dem 18. Lebensmonat. Sie können als maximale motorische Funktion das selbständige 
Stehen erreichen. Ein Erkrankungsbeginn nach dem 21. Lebensjahr spricht für eine SMATyp IV mit geringen motorischen Einschränkungen (Byers und Banker 1961; Russman et al. 1992; Russman 2007; Kolb und Kissel 2011). Trotz des defekten SMN1-Gens besitzen die Patienten intakte SMN2-Genkopien. Arakawa et al. (2016) zeigten, dass der Phänotyp schwerwiegender ausgeprägt ist, je weniger SMN2-Kopien vorliegen (Lefebvre et al. 1997; Arakawa et al. 2016). Neue medikamentöse Therapien, zum Beispiel Nusinersen (Finkel et al. 2017; Pechmann et al. 2019), machen sich die Expression dieser SMN2-Kopien zu Nutze (Burnett et al. 2009; Mercuri et al. 2012; Shorrock et al. 2018).

Das Hauptaugenmerk der Kinderorthopäden liegt bisher bei der SMA-Typ II und III, bei denen durch die supportive Therapie die Lebensqualität verbessert werden kann. Patienten mit SMA-Typ IV zeigen eine milde Ausprägung der Symptome, die sich konservativ führen lässt (Fujak et al. 2013). Die SMA-Typen II und III leiden mit steigendem Alter an einer zunehmenden generellen Muskelschwäche. Beginnend mit der Schwäche der proximalen Muskulatur entwickeln sich im Verlauf Symptome des Bewegungsapparates wie Skoliosen, Beckenschiefstände, Kontrakturen, Hüftluxationen und Osteoporose (Sporer und Smith 2003; Castro und Iannaccone 2014; Putzier et al. 2016). Für die Patienten bedeutet das ein Leben im Rollstuhl und einen Alltag, in dem sie kontinuierlich auf Hilfe angewiesen sind. Außerdem leiden sie unter Begleiterkrankungen (Komorbiditäten) wie restriktive Lungenerkrankungen mit Gefahr von TIS und an Ernährungsproblemen bis hin zur Unterernährung (Kachexie). Hingegen sind Sensibilität und mentale Fähigkeiten nicht beeinträchtigt (Evans et al. 1981; Lunn und Wang 2008; McElroy et al. 2011; Markowitz et al. 2012; Mercuri et al. 2012).

Dass die SMA fast zwingend mit der frühkindlichen Skoliose einhergeht, zeigen Zahlen aus folgenden Studien: Die Inzidenz einer neuromuskulären Skoliose bei SMA liegt insgesamt betrachtet bei $70 \%$ (Canavese et al. 2014), bei schwerwiegenden Fällen sogar bei $100 \%$ (Chandran et al. 2011), weshalb die Patienten schon früh auf eine dauerhafte, mitwachsende Stütze angewiesen sind (Lunn und Wang 2008). Typischerweise sind es weiche Skoliosen mit langen flexiblen Krümmungsbögen, die unter die neuromuskulären EOS eingeteilt werden können (siehe Abb. 1) ( Schwentker und Gibson 1976; Carstens 1999; Chandran et al. 2011; Putzier et al. 2016; Rumalla et al. 2016). 

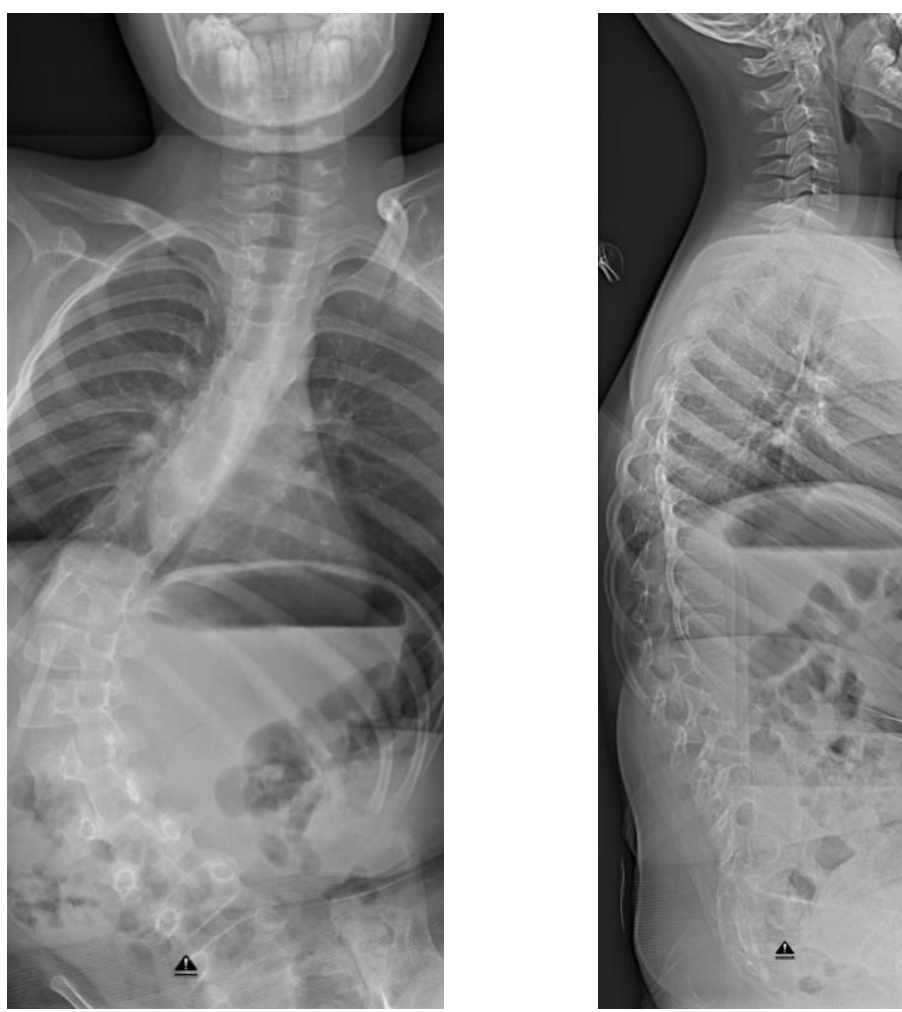

Abbildung 1: Röntgenbilder eines SMA-Patienten im a.p. und seitlichen Strahlengang. Zu sehen ist die langstreckige rechtskonvexe lumbale Wirbelsäulenverkrümmung eines 10-jährigen SMA-Typ-II-Patienten.

Die Therapie der Skoliose bei neuromuskulärer EOS ist eine Herausforderung. Durch die pulmonalen Komorbiditäten der an SMA erkrankten Kinder ist es kontraproduktiv, ihren Raum zum Atmen mit einem Korsett noch weiter einzuengen, sodass in der Regel eine operative Therapie indiziert ist. Ziel ist es dabei, durch eine Aufrichtung der Wirbelsäule die Sitzfähigkeit der Patienten zu verbessern und Platz im Thorax für die Lungenentwicklung zu schaffen. Eine bessere Lungenentwicklung und Lungenfunktion beugt einem TIS vor. Insgesamt wird so die Lebensqualität der Patienten verbessert (Campbell et al. 2003; Campbell et al. 2004; Gillingham et al. 2006; Bowen et al. 2008; Chandran et al. 2011). Gleichzeitig muss im perioperativen Management beachtet werden, dass SMA-Kinder vor allem wegen der schlechten Lungenfunktion und der geringen Belastbarkeit als Hochrisikopatienten für chirurgische Interventionen eingestuft werden und vermehrt von postoperativen Komplikationen betroffen sind. Ganz ohne Intervention nehmen allerdings die Wirbelsäulendeformität und die damit verbundenen Einschränkungen und Probleme während des Wachstums zu. Ein gutes interdisziplinäres Management ist für ein gutes Ergebnis notwendig (Haaker und Fujak 2013; Canavese et al. 2014).

In Bezug auf die therapeutischen Maßnahmen bei der SMA lassen sich zwei Säulen unterscheiden. Die Therapie der SMA basiert einerseits auf der Säule der multidisziplinären supportiven Maßnahmen und andererseits neuerdings auf der zweiten Säule der 
medikamentösen ursächlichen Therapie. Die Kinderorthopädie stellt dabei einen Teil der supportiven Therapie dar, der konservative und operative Maßnahmen zur Besserung von orthopädischen „Baustellen“, wie zum Beispiel Skoliose, Kontrakturen und Hüftgelenksluxationen beinhaltet.

\subsection{Therapieoptionen}

Es gibt verschiedene operative Therapieoptionen bei der Skoliosen-Krümmungskorrektur im Wachstumsalter. Die modernen orthopädischen Therapieverfahren verzichten auf eine frühe Spondylodese. Zurzeit kommen vor allem distrahierbare, sogenannte „mitwachsende“ Implantate zum Einsatz (Granata et al. 1989; Chandran et al. 2011; Tis et al. 2012). Pharmakologisch und pulmologisch bestehen sehr unterschiedliche Therapieoptionen für die Behandlung der SMA, welche insbesondere vom Schweregrad der Erkrankung abhängen. Es wird im Folgenden auf die für diese Studie relevanten Implantate und auf weitere relevante SMA-Behandlungen eingegangen.

\subsubsection{Skoliosetherapie VEPTR ${ }^{\circledR}$}

Unter den mitwachsenden Implantaten für die EOS ist eine Therapievariante das Verticalexpandable-prosthetic-titanium-rib-Implantat (VEPTR®, Firma Synthes)(Hell et al. 2005). Dabei handelt es sich um teleskopierbare Titan-Rippen, welche je nach Indikation auf unterschiedliche Art und Weise zur Anwendung kommen. Im Falle von SMA-Patienten hat sich bewährt, die VEPTR ${ }^{-S t a n g e n ~ a u f ~ b e i d e n ~ S e i t e n ~ n e b e n ~ d e r ~ W i r b e l s a ̈ u l e ~ z w i s c h e n ~}$ kranialen Rippen und dem Becken mittels entsprechender Haken aufzuspannen und zu befestigen (Eifeltower Construction). Mit dieser Konstruktion kann einerseits eine Verringerung der Skoliosekrümmung und eine Verbesserung der Beckenkippung erzielt werden, und andererseits kann durch die Aufrichtung der Rippen mehr Platz im Thorax geschaffen werden (Hell et al. 2005; Groenefeld und Hell 2013; Haaker und Fujak 2013). Es ist nachgewiesen, dass der zusammengesackte verdrehte Thorax der SMA-Patienten der Lunge wenig Raum lässt sich zu entwickeln und zu Lasten der Lungenfunktion geht sowie die Gefahr eines TIS birgt (Campbell et al. 2003). Die Thorax- und Wirbelsäulenaufrichtung durch das Implantat erreicht eine verbesserte Lungenfunktion und beugt einem TIS vor (Chua et al. 2016). Außerdem kann ein VEPTR®-Implantat mittels eines mechanisch distrahierbaren Teleskopteils dem kindlichen Wachstum angepasst werden. Dazu ist halbjährlich eine 30 bis 45-minütige Operation unter Vollnarkose zur Aufdehnung erforderlich.

Neben den vielen Vorteilen des VEPTR ${ }^{\circledR}$-Systems sind auch Komplikationen beschrieben. Einerseits sind das die operationsassoziierten Komplikationen der halbjährlichen Operationen unter Vollnarkose, wie häufige Infekte (Plaass et al. 2016; Wagner et al. 2018), oder es handelt sich um Probleme im Rahmen der Anästhesie (Islander 2013). Auf der anderen Seite führen Vernarbungs-, Ossifikations- und Autofusionsprozesse dazu, dass 
sich jenseits von vier Jahren Behandlung trotz weiterer Distraktionsoperationen der CobbWinkel zunehmend wieder verschlechtert (law of diminishing returns)(Sankar et al. 2011; Fletcher und Bruce 2012; Groenefeld und Hell 2013; Zivkovic et al. 2014). Als einen Ausblick beschreiben Fletscher und Bruce im Jahr 2012 einen Verbesserungsvorschlag wie folgt: "Future options may include fusionless techniques that do not require multiple surgical lengthenings"(Fletcher und Bruce 2012). Eine solche Therapie, welche Autofusion und Operationen vermeiden möchte, wird im Folgenden beschrieben.

\subsubsection{Skoliosetherapie MAGEC®}

Als neue Therapiemöglichkeit der EOS als Alternative zum VEPTR®-System wurden die magnetically controlled growing rods (MCGR) im Jahr 2009 weltweit erstmalig an der Universitätsklinik in Hong Kong verwendet (Cheung et al. 2012). Die MCGRs wurden wirbelsäulennah mit Pedikelschrauben befestigt und konnten im Gegensatz zu VEPTR®-Stangen nicht invasiv mithilfe eines elektrischen Magneten im ambulanten Rahmen verlängert werden. In Deutschland wurde im Jahr 2011 an der Universitätsmedizin in Göttingen (UMG) das Magnetic-Expansion-Control (MAGEC®, Firma Ellipse, jetzt NuVasive)-System mit VEPTR ${ }^{\circledR}$-Fixierungen kombiniert mit dem Ziel, eine möglichst flexible Wirbelsäule zu erhalten und Ossifikations- und Autofusionsprozesse zu minimieren (siehe Abb.2)( Hell et al. 2018). Das System wird dem physiologischen Wachstum angepasst und im Abstand von jeweils drei Monaten um fünf Millimeter extern verlängert. Derzeit ist die Therapie von neuromuskulären EOS mit den paravertebralen beidseitigen MAGEC®-Stangen eine anerkannte Option. Besonders erfolgreich ist der Einsatz bei weichen Skoliosen, wie die der SMA-Patienten, wobei die Langzeitergebnisse sowie die Untersuchung eines law of diminishing returns noch ausstehen (Lorenz et al. 2017).

Die operationsbedingten Komplikationen scheinen vergleichbar mit denen der VEPTR ${ }^{\circledR}$ Therapie (Aslan et al. 2019; Bekmez et al. 2019) mit dem Unterschied, dass durch die drastische Verminderung der Anzahl der Operationen sich folglich die totale Anzahl dieser operativ bedingten Komplikationen bei der MAGEC®-Therapie auch vermindert. Limitationen des Systems ergeben sich einerseits durch die eingeschränkte Magnetresonanztomografie (MRT) Kompatibilität des Implantates und andererseits durch technische Grenzen. So wird bei zu viel Druck und Spannung auf dem System ein Ausfahren des Stangengewindes unmöglich, womit eine Anwendung bei sehr adipösen Kindern oder sehr steifen Skoliosen schwierig wird (Lorenz et al. 2019). 


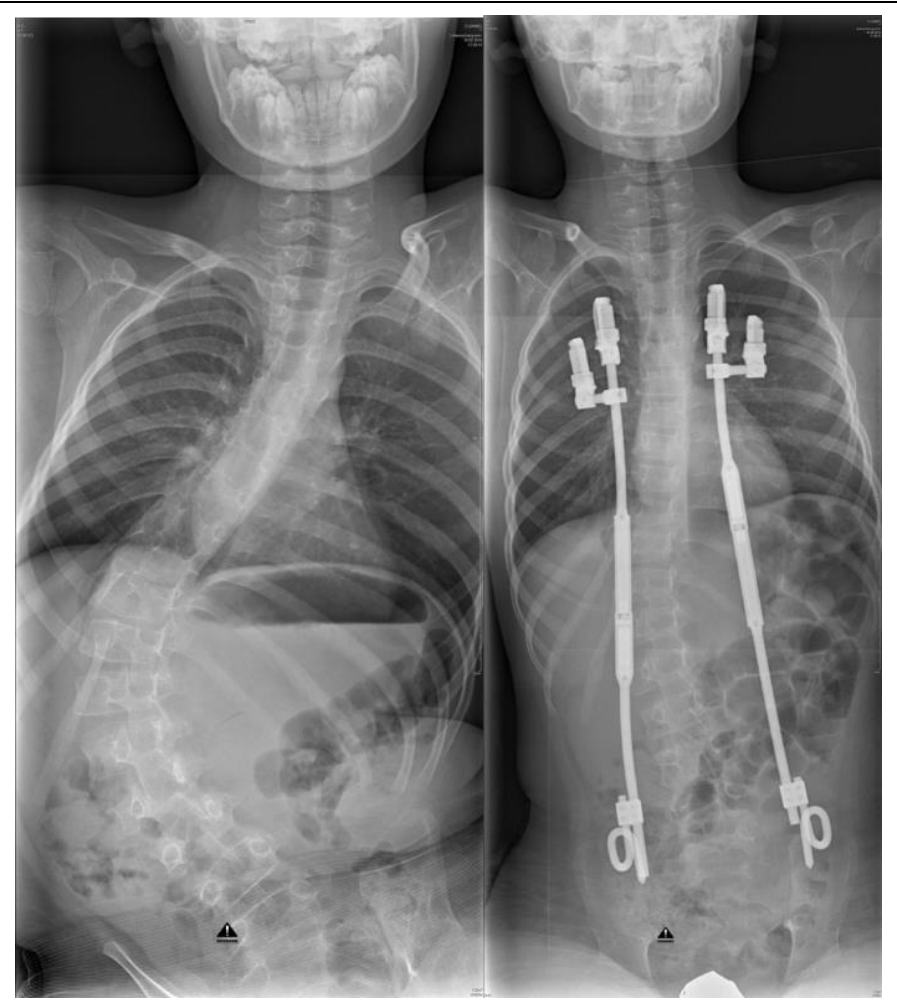

Abbildung 2: Röntgenbilder eines SMA-Patienten im a.p. Strahlengang. Zu sehen sind die Situationen Prä- und Postimplantation des MAGEC®-Systems mit VEPTR®-Haken-Fixierung und die dadurch erreichte Krümmungskorrektur bei einem 10-jährigen SMA-Typ-II-Patienten.

\subsection{Therapieoptionen für SMA}

Die Therapie der SMA besteht aus der kausalen Therapie und der symptomatischen Therapie in interdisziplinärer Zusammenarbeit (Burnett et al. 2009; Haaker und Fujak 2013; Canavese et al. 2014; Castro und Iannaccone 2014; Waldrop und Kolb 2019).

\subsubsection{Symptomatische Therapie der Skoliose bei SMA}

Die Symptomatik der SMA-Patienten ergibt sich durch die Folgen der immer schwächeren Muskulatur. Je nach SMA-Typ unterscheiden sich die Beschwerden. Während der Großteil der Patienten sich nicht selbst in der Sitzposition halten kann und eine Skoliose bekommt (Typ I und II), können Patienten mit Typ III zumeist laufen.

Um zu entscheiden, welcher Patient von einer operativen Therapie der neuromuskulären Skoliose profitiert, haben Canavese et al. (2014) Faktoren für eine Operationsindikation aufgestellt. Dazu zählen: Funktionelle und/oder kardio-respiratorische Störungen, fehlende Effizienz einer Orthesentherapie, eine unvermeidbare Progression, obwohl nur ein Stadium geringer Krümmung vorliegt, und Deformitäten, die Schmerzen bereiten (Canavese et al. 2014). 
Für SMA-Patienten mit entsprechender Indikation hat sich die frühzeitige Therapie mit dem MAGEC®-System zumindest im Zwei-Jahres-Follow-up bewährt (Lorenz et al. 2017). Durch die Aufrichtung der Wirbelsäule sowie der Ermöglichung von Wirbelsäulenwachstum und der Schaffung von Platz im Thorax zur Verbesserung der Lungenentwicklung wird erzielt, dass weniger Komorbiditäten auftreten und damit voraussichtlich eine längere Lebenserwartung mit mehr Lebensqualität gewonnen wird. Das Therapieende der mitwachsenden Implantate ergibt sich aus dem Wachstumsabschluss der Kinder. Eine Wirbelsäulenversteifung (Spondylodese) fixiert das Krümmungskorrekturergebnis und sichert die Haltungsunterstützung im weiteren Verlauf (Thompson et al. 2016).

\subsubsection{Kausale Therapie der SMA mit Nusinersen (Spinraza $\left.{ }^{\circledR}\right)$}

Nusinersen, auch unter dem Handelsnamen Spinraza ${ }^{\circledR}$ (Firma Biogen) bekannt, ist ein neues Medikament zur Therapie der SMA. Nach sehr erfolgreichen Zulassungsstudien und der Empfehlung der Europäischen Arzneimittelagentur (EMA) von Spinraza ${ }^{\circledR}$, wurde das Medikament zum Juni 2017 für SMA-Typen I bis III in Deutschland zugelassen (Biogen 2017) und die Entwicklung mit fortführenden Studien wie der SMArt-CARE-Study weiter verfolgt (Finkel et al. 2017; Pechmann et al. 2019).

Vor einer Nusinersen-Therapie wird ein SMA-Patient genetisch untersucht. Es wird die Anzahl der SMN2-Kopien festgestellt, denn an die prä-mRNA dieses Gens bindet das Medikament, spleißt sie und steigert die Expression von funktionellem SMN-Protein. Der klinische Effekt war bei sehr früh behandelten SMA-Typ-I-Patienten ein längeres Überleben und das Erreichen von motorischen Meilensteinen, wie zum Beispiel Kopflontrolle oder Sitzfähigkeit (Finkel et al. 2017; Shorrock et al. 2018; Pechmann et al. 2019).

Das Medikament muss intrathekal appliziert werden. Im deutschsprachigen Raum werden dafür alle vier Monate Lumbalpunktionen durchgeführt. Zur Applikation des Medikamentes ist eine möglichst normale Wirbelsäulenanatomie von Vorteil, wenn nicht sogar Voraussetzung. Die in dieser Arbeit untersuchte Operationsmethode mit den paravertebralen Implantaten lässt einen freien Zugangsweg zur Lendenwirbelsäule ohne heterotope Ossifikationen zu im Gegensatz zu der bereits beschriebenen Methode, die MAGEC®-Stangen mittels Pedikelschrauben direkt auf der dorsalen Wirbelsäule zu fixieren. Im Rahmen der definitiven Versorgung nach Wachstumsabschluss in Form einer Spondylodese, sollte intraoperativ ein Fenster in der dorsalen ossären Fusion geschaffen werden, um die Lumbalpunktionen weiter zu ermöglichen (Lorenz et al. 2018) oder ein Katheter eingelegt werden (Strauss et al. 2018). 
Derzeit werden große Hoffnungen in dieses und ähnliche Medikamente zur ursächlichen Behandlung der SMA gesetzt. Alternativ wird an einer Genersatztherapie geforscht (Bozorg Qomi et al. 2019).

Mit Spannung werden Langzeitstudien erwartet, um den tatsächlichen Verlauf der verschiedenen SMA-Typen unter Spinraza ${ }^{\circledR}$ - oder Genersatztherapie beobachten zu können (Mercuri et al. 2018; Shorrock et al. 2018; Waldrop und Kolb 2019).

\subsection{Aktueller Wissensstand in der Forschung}

Im Jahr 2019 kommentierten Hosseini und Akbarnia die Studie „Mean 6-Year Follow-up of Magnetically Controlled Growing Rod Patients With Early Onset Scoliosis: A Glimpse of What Happens to Graduates.“ (Cheung et al. 2019) und stellten fest: Es fehlen Langzeitstudien zu MAGEC®-Implantaten, es gibt ein wissenschaftliches Interesse an dem Abschluss der MAGEC®-Behandlung, es werden Studien zu Komplikationen und Effizienz von MAGEC®-Implantaten im Langzeitverlauf gebraucht und neben technischen Daten sind auch psychosoziale Aspekte von Interesse und bislang noch nicht untersucht (Hosseini und Akbarnia 2019). Die vorliegende Arbeit geht auf die ersten drei der vier genannten Aspekte ein.

Darüber hinaus wird sowohl für VEPTR®- als auch für MAGEC®-Systeme in aktuellen Studien ein law of diminishing returns beschrieben, was bedeutet, dass trotz Fortführung der Therapie in Form von gleicher Art, Frequenz und Ausmaß der Distraktionen, der Effekt auf die Wirbelsäulenkrümmung Jahr für Jahr abnimmt und sich die Krümmung somit ab einem Punkt zunehmend verschlechtert (Sankar et al. 2011; Ahmad et al. 2017; Lampe et al. 2019; Studer et al. 2019; Wu et al. 2019). Studer et al. (2019) beschrieb dieses Phänomen bereits nach zwei Jahren MAGEC®-Therapie mit wirbelsäulennaher Fixierung. Aufgrund der Tatsache, dass das law of diminishing returns offensichtlich negative Auswirkungen für die Patienten bedeutet, ist es hoch relevant, in Studien einen Fokus auf dieses Phänomen, deren mögliche Ursachen und mögliche Lösungen zu legen.

Die prospektive Beobachtung von einem homogenen Kollektiv mit SMA und MAGEC®Therapie von mehr als vier Jahren ist zum gegenwärtigen Zeitpunkt in dieser Arbeit einmalig und grundsätzlich sind homogene Patientenkollektive sehr geeignet, um systematische Fehler durch Kollektivinhomogenitäten $\mathrm{zu}$ verringern und die Effekte einzelner Methoden oder Faktoren genau beurteilen zu können. Ebenso ist der Effekt einer vorausgegangenen MAGEC®-Behandlung auf das Endergebnis der dorsalen Spondylodese von großem Interesse. 


\subsection{Fragestellung}

In der vorliegenden Arbeit wurden die Langzeitergebnisse der operativen Skoliosetherapie mit paravertebral implantierten, perkutan distrahierbaren Wirbelsäulenimplantaten an einem homogenen, prospektiv eingeschlossenen SMA-Kinderpatientenkollektiv mit Wirbelsäulendeformitäten untersucht. Es wurden zwei Fragestellungen bearbeitet.

Zum einen die Fragestellung: „Wie verhält sich die Wirbelsäulenkrümmung bei SMAKindern, die mit bilateralen MAGEC®-Implantaten versorgt wurden, im Langzeitverlauf? Wird in dem prospektiv untersuchten, homogenen Kollektiv ein law of diminishing returns beobachtet?"

Und weiterhin: „Haben SMA-Kinder, welche mit MAGEC ${ }^{\circledR}$ - oder VEPTR ${ }^{\circledR}$-Implantaten über Jahre behandelt wurden, bessere Ergebnisse nach der endgültigen Spondylodese als unbehandelte SMA-Jugendliche?“ 


\section{Material und Methoden}

\subsection{Ethikkommission}

Das Studienprotokoll mit dem Arbeitstitel „Die Behandlung der kindlichen Skoliose bei spinaler Muskelatrophie mit extern zu kontrollierenden magnetischen Implantaten“ wurde durch die Ethikkommission der medizinischen Fakultät der Georg-August-Universität Göttingen geprüft und am 17.08.2015 mit der Antragsnummer DOK_246_2015 genehmigt.

\subsection{Studiendesign}

Seit Dezember 2011 wird eine prospektive Kohortenstudie vom kinderorthopädischen Schwerpunkt der Universitätsmedizin Göttingen unter Leitung von Frau Prof. Dr. AnnaKathrin Hell an einem Studienkollektiv von Kindern mit SMA, Wirbelsäulendeformitäten und MAGEC®-Implantaten beziehungsweise dorsaler Spondylodese durchgeführt.

\subsection{Patientenkollektiv}

Primär wurden alle von SMA betroffenen Kinder und Jugendlichen, die im Laufe ihrer Erkrankung eine therapiebedürftige, neuromyopathische Skoliose entwickelt hatten, in die prospektive Untersuchung aufgenommen. Patienten wurden erfasst, die mit einem MAGEC®-Implantat beidseits der Wirbelsäule oder einer Wirbelsäulenversteifung in der Zeit von 2011 bis Dezember 2018 in der Universitätsmedizin Göttingen versorgt wurden. Als Beginn der Beobachtung wurden die letzte Konsultation und das Röntgenbild vor der Primärimplantation festgelegt. Der Endpunkt für die Beobachtung in dieser Arbeit war die erste Konsultation und Röntgenbild nach einer Wirbelsäulenversteifung bzw. eine Mindestbeobachtungszeit von vier Jahren nach MAGEC®-Implantation und/oder Verlaufsbeobachtungszeit bis Dezember 2018.

Zur Auswertung der spezifischen Fragestellungen wurde das gesamte Patientenkollektiv in folgende Untergruppen eingeteilt:

Für die Auswertung der Fragestellung 1 wurden 17 SMA-Patienten in die Kohorte 1 eingeschlossen, die an einer neuromuskulären Skoliose erkrankt waren, mit MAGEC®-Implantaten beidseits der Wirbelsäule versorgt, und länger als vier Jahre beobachtet wurden. Die mittlere Beobachtungszeit betrug 4,6 Jahre. Von diesen 17 SMA-Kindern wurden 15 Patienten ausschließlich mit dem MAGEC®-Implantat behandelt. Zwei Kinder waren mit

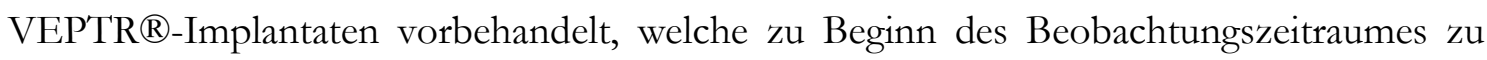
MAGEC®-Implantaten ausgetauscht wurden. 
Aus dieser Kohorte konnten neun Patienten vom Beginn bis zum Endpunkt der Therapie verfolgt werden, das heißt, von der Primärimplantation bis zur Spondylodese.

Zur Analyse der Fragestellung 2 wurden Jugendliche mit SMA und Wirbelsäulendeformität in die Kohorte 2 eingeschlossen, welche in der Pubertät eine Spondylodese Operation erhielten. Die 14 Patienten der Kohorte 2a waren nicht vorbehandelt. Ebenfalls 14 Patienten in der Kohorte $2 \mathrm{~b}$ hatten während der Wachstumsphase mitwachsende Implantate, wobei acht Patienten mit MAGEC®-Implantaten behandelt wurden, drei Patienten mit VEPTR ${ }^{\circledR}-$ Implantaten und drei Patienten mit VEPTR ${ }^{\circledR}-$ Implantaten mit Umbau zu MAGEC®-Implantaten im Verlauf.

\subsection{Therapieverlauf}

Der Therapieverlauf der Kohorte 1 lässt sich wie folgt beschreiben:

$\mathrm{Zu}$ Beginn der Therapie wurde den Patienten in einer primären Operation beidseits der Wirbelsäule das MAGEC®-Implantat eingesetzt (siehe 2.4.1). Mit Ausnahme von zwei Patienten, bei denen die VEPTR®-Stangen zu MAGEC®-Stangen getauscht wurden (siehe Abb. 3).

Nach fünf Monaten schloss sich postoperativ die erste externe Verlängerung an (siehe 2.4.2). Alle weiteren externen Verlängerungen wurden im Anschluss in einem Intervall von ca. drei Monaten fortgeführt, um die Zunahme der Wirbelsäulenlänge durch kurze Intervalle einem möglichst physiologischen Wachstum anzugleichen. Sie konnten in einem ambulanten Rahmen erfolgen.

Die Verlängerungsstrecke des magnetisch ausfahrbaren Teleskopteils des Implantates reichte je nach individueller Wachstumsgeschwindigkeit des Kindes ca. zwei bis drei Jahre. Folglich wurde nach dieser Zeit für die Patienten mit komplett ausgefahrenem Implantat eine Wechseloperation geplant (siehe 2.4.3). Individuell traf der Operateur die Entscheidung, nur die Stangen zu tauschen, zusätzlich einen oder mehrere Haken zu wechseln oder zusätzliche Ausleger an die Rippenhaken anzubringen, um beispielsweise den erhöhten Druck auf das System aufgrund von Übergewicht des Patienten auf eine größere Auflagefläche zu verteilen.

Im Anschluss erfolgte erneut eine Phase der externen Verlängerungen des gewechselten Implantates mit einem ersten Termin fünf Monate postoperativ und im weiteren Verlauf Verlängerungen im Intervall von ca. drei Monaten für weitere ca. zwei bis drei Jahre (siehe 2.4.4).

Mit dem Einsetzen der Pubertät, bei starker Kurvenprogression oder nach dem Abschluss der Hauptwachstumsphase der Kinder wurde ein mitwachsendes Implantat obsolet und die Indikation zur Wirbelsäulenversteifung (Spondylodese) gestellt (siehe 2.4.5). Zusätzlich zu der durch die MAGEC®-Therapie erreichte Krümmungsverbesserung konnte im Rahmen der Spondylodeseoperation die Krümmung weiter reduziert und auf lange Sicht gesichert 
werden. Dieser Abschluss der MAGEC®-Therapie entsprach gleichzeitig dem Abschluss der Studie und wurde als Endpunkt für Messungen und Recherche gewertet.

Parallel zu der Skoliosetherapie bekamen seit der Zulassung von Spinraza® im Juni 2017 nach und nach mehr Patienten des Kollektivs das Medikament (siehe 2.4.6). Das Schema begann mit einer Aufdosierungsphase, an die sich eine Erhaltungsphase von intrathekalen Medikamentengaben anschloss. Um bei Wirbelsäulendeformität und/oder Wirbelsäulenversteifung weiterhin eine intrathekale Injektion $\mathrm{zu}$ ermöglichen, wurde bei der Spondylodese aktiv ein Loch geschaffen (Lorenz et al. 2018).

\section{Kohorte 1}

\begin{tabular}{|c|c|}
\hline VEPTR ${ }^{\circledR}$-Therapie $\rightarrow$ & MAGEC®-Therapie $(\mathrm{n}=2)$ \\
\hline
\end{tabular}

\begin{tabular}{|c|c|c|}
\hline & MAGEC®-Therapie $(\mathrm{n}=15)$ & | Spondylodese $(\mathrm{n}=7)$ \\
\hline | Beginn & $\varnothing$ 4,6 Jahre Verlaufsbeobachtung & | Ende \\
\hline
\end{tabular}

Abbildung 3: Skizze des Therapieverlaufes der Kohorte 1. Hier sind die 17 Kinder aufgeführt, die eine Verlaufsbeobachtungszeit von über vier Jahren während der MAGEC®-Behandlung erreichten.

Zum Therapieverlauf der Kohorte 2 (siehe Abb. 4) lässt sich ergänzend sagen:

Der Therapieverlauf der acht Patienten mit MAGEC®-Vorbehandlung aus der Kohorte 2b entsprach dem für die Kohorte 1 beschriebenen Therapieverlauf. Drei Patienten aus der Kohorte $2 \mathrm{~b}$ wurden mit VEPTR ${ }^{\circledR}$ vorbehandelt und ebenfalls 3 Patienten begannen die VEPTR ${ }^{\circledR}$-Behandlung, wechselten jedoch im Verlauf zum MAGEC®-Implantat. Die Operationstechnik des VEPTR ${ }^{\circledR}$-Systems gleicht dem Vorgehen der MAGEC®Implantation bis auf den teleskopierbaren mittleren Implantatteil. Als Fixierungspunkte 
wurden jeweils die Rippen- und Beckenhaken des VEPTR ${ }^{\circledR}-S y s t e m s$ verwendet. Die chirurgischen Verlängerungen erfolgten im Abstand von sechs Monaten im Rahmen von 30 bis 45-minütigen operativen Eingriffen. Die Patienten der Kohorte 2a wurden konservativ geführt, vernachlässigt oder stellten sich aus den unterschiedlichsten Gründen (z.B. späte Auswanderung nach Deutschland) erst in der Pubertät zu einer Behandlung vor. Diese Patienten waren jenseits des Alters oder der Gewichtszulassung für wachstumsfreundliche Implantate, sodass Kohorte 2 direkt nach sorgfältigen präoperativen Abklärungen eine dorsale Spondylodese erhielt (siehe 2.4.5).

\section{Kohorte 2}

Kohorte 2a

Kohorte $2 \mathrm{~b}$

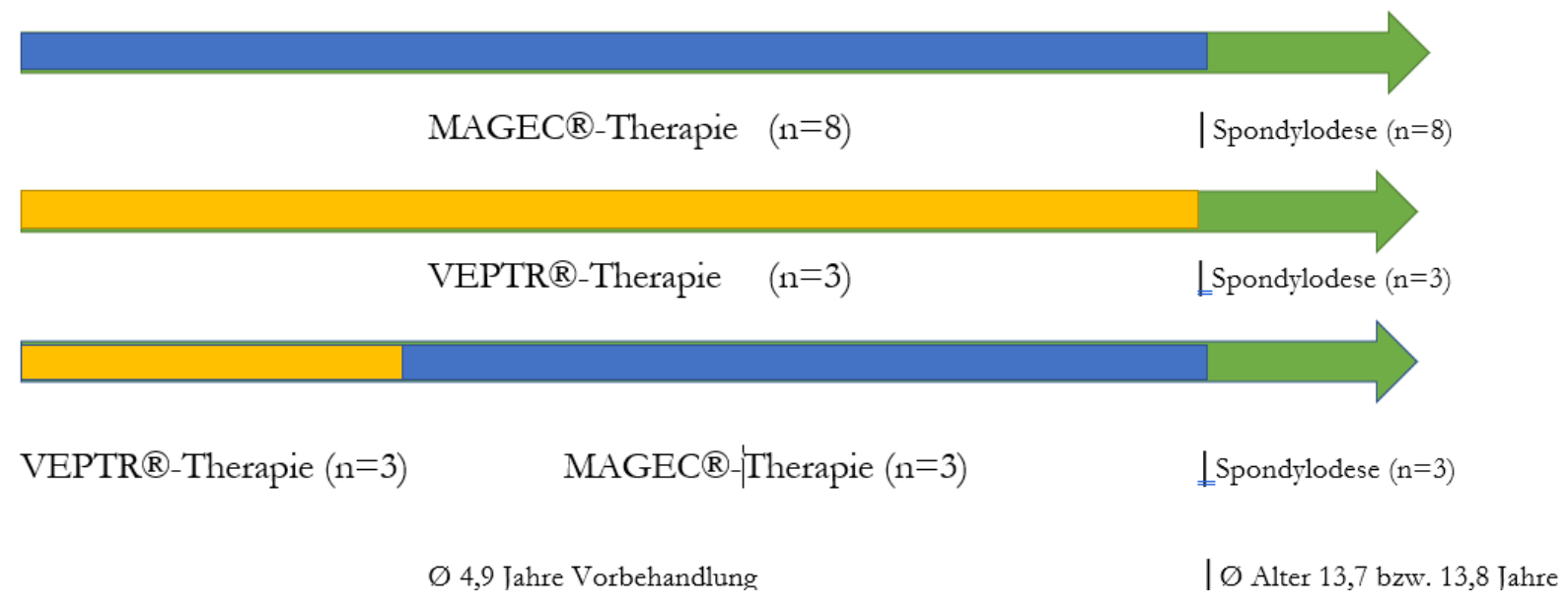

Abbildung 4: Skizze des Therapieverlaufes der Therapie der Kohorte 2. Hier erhielten alle SMAPatienten eine dorsale Spondylodese. Kohorte 2a hatte im Vorfeld keine operative Therapie erhalten, während Kohorte $2 \mathrm{~b}$ entweder mit MAGEC®- oder mit VEPTR ${ }^{\circ}$-Implantaten im Mittel über 4,9 Jahre vorbehandelt wurde. 


\subsubsection{Primärimplantation}

Zunächst erfolgten Voruntersuchungen zur Diagnostik und Operationsplanung. Intraspinale oder kraniale Prozesse, die MRT-Verlaufskontrollen erfordern, stellten eine Kontraindikation für die Magnetimplantate dar.

Um einen Überblick über das Operationsverfahren zu geben, werden im Folgenden wichtige Eckpunkte dargestellt. In der Arbeit von Katharina Grönefeld aus dem Jahr 2016 wurde die Operationstechnik zur Kombination von VEPTR ${ }^{\circledR}-$ Haken und MAGEC $\AA$ Stangen, wie sie bei den Patienten des Studienkollektivs auch angewandt wurde, genau beschrieben (Groenefeld und Hell 2016; Hell et al. 2018).

Die MAGEC®-Implantation wurde in Bauchlage unter Allgemeinnarkose vorgenommen. Es wurde mit der konkaven Seite begonnen. Die Rippenhaken wurden idealer Weise proximal der kranialen Krümmung im Bereich der dritten bis fünften Rippen implantiert. Die Beckenkammhaken wurden durch eine Muskellücke durch die Rumpfmuskulatur hindurch auf die Beckenkämme gesteckt. Beide Fixierungspunkte sollen Rumpfbewegungen ermöglichen. Mit einer Rollenbiegezange wurden die MAGEC ${ }^{\circledR}-$ Implantate dem sagittalen Profil angepasst und nach vorheriger Funktionsprüfung unter dem dorsalen Weichteilmantel hindurch zwischen den Rippen- und Beckenhaken aufgespannt. Auf die gleiche Weise folgte die Implantation auf der konvexen Seite (siehe Abb. 5). Schließlich wurde die Operation mit dem mehrschichtigen Wiederverschluss der Inzisionen beendet.

Zu dem postoperativen Management gehörte eine sitzende oder stehende Kontrollröntgenaufnahme in zwei Ebenen, um eine regelrechte Implantatlage sicherzustellen. Diese Aufnahme konnte zur Analyse in dieser Studie genutzt werden. Nach circa einer Woche konnten die Patienten bei reizlosen Wundverhältnissen, Beschwerdefreiheit und sicherer Mobilisation in die weitere ambulante Behandlung entlassen werden. 


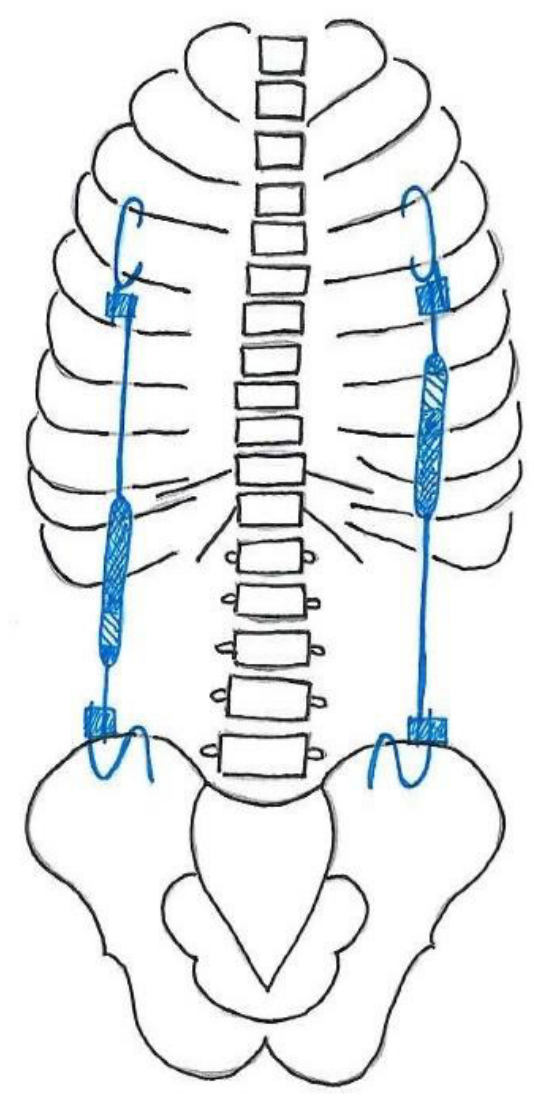

Abbildung 5: Skizze des MAGEC®-Implantates mit VEPTR®-Haken-Befestigung.

\subsubsection{Externe Verlängerungen}

Zur ersten externen Verlängerung kamen die Patienten fünf Monate nach der Operation, danach im Abstand von ca. drei Monaten zu allen weiteren Verlängerungen. Die kurzen Intervalle sollten einem möglichst physiologischen Wirbelsäulenwachstum entsprechen. Bei dem ambulanten Verlängerungstermin wurden die Kinder erst gewogen und gemessen und anschließend in Bauchlage mit einem entsprechenden Gerät (MAGEC external remote controller $\left.{ }^{\circledR}, \mathrm{NuVasive}\right)$ durch Magneten von extern verlängert. Dazu ertastete der behandelnde Arzt zunächst das Implantat und suchte mit einem kleinen Magneten (MAGEC Locator ${ }^{\circledR}$, NuVasive) den magnetischen Bereich des Teleskopteils auf. Dieser wurde auf der Haut markiert und das Verlängerungsgerät auf die Markierung aufgesetzt. Die Verlängerungsstrecke wurde für den Großteil der Patienten auf fünf Millimeter ( $\mathrm{mm}$ ) festgelegt. Diese Strecke wurde anhand von Anhaltspunkten wie Schmerzen nach der letzten Verlängerung, die nach übermäßigen Verlängerungen auftreten können, von Schmerzen vor der aktuellen Verlängerung, vom Krümmungsverlauf und dem Einrasten des Implantates bei maximaler Spannung individuell um wenige Millimeter angepasst. Um den 
Druck auf das Implantat zu verringern und die Umdrehungen des Teleskopteils zu erleichtern, war es vor allem bei adipösen Patienten notwendig, die Verlängerung in Seitenlage über ein Hypomochlion zur Aufdehnung der Krümmung durchzuführen. Während der Verlängerung kontrollierte der Arzt die Verlängerungsstrecke über die Anzeige des Gerätes. Da die Patienten unter der Verlängerung keine Schmerzen beklagten, sondern nur ein auszuhaltendes Gefühl von leichter Vibration beschrieben, war eine Narkose für die Intervention nicht notwendig. Abschließend wurde ein sitzendes Röntgenbild in zwei Ebenen aufgenommen, um das komplikationsfreie Ausfahren des Implantates und die regelrechte Implantatlage beurteilen und bestätigen zu können. Diese Röntgenbilder konnten ebenfalls zur Auswertung des Krümmungsverlaufes genutzt werden.

\subsubsection{Austausch-Operation}

Die beschriebenen Implantate stellten ein Überbrückungsverfahren während des Wirbelsäulenwachstums dar. Sollten die Implantate vor Erreichen des Alters für eine dorsale Spondylodese vollständig ausgefahren sein, wurden sie ausgetauscht. Zu diesem Termin erfolgte eine stationäre Aufnahme. Vergleichbar zu der Vorgehensweise der Primärimplantation fand auch diese Operation in Bauchlage unter Allgemeinanästhesie statt. Das Ziel der Operation war meist nur ein Wechsel der ausgefahrenen Stangen. Je nach Eigenschaften des jeweiligen Patienten traf der Operateur individuelle Entscheidungen zu einem zusätzlichen Tausch der Rippenhaken, beispielsweise aufgrund von Dislokation, oder zu einem Hinzufügen von Auslegern zur Druckverteilung bei adipösen Patienten.

Die Operationstechnik entsprach der für die Primärimplantation in 2.4.1 beschriebenen Vorgehensweise. Das postoperative Prozedere lässt sich ebenfalls vergleichen.

\subsubsection{Externe Verlängerungen des Austauschimplantates}

Auch bei den externen Verlängerungen des gewechselten Implantates kamen die Patienten erst fünf Monate postoperativ, dann alle drei Monate zur ambulanten externen Verlängerung in die Klinik. Vom Ablauf glichen die Termine denen des ersten Implantates und fanden in der in 2.4 .2 beschriebenen Art und Weise statt. Als Unterschiede könnte man voroperierte, ältere, größere und damit auch schwerere Kinder benennen, wobei dies, ausgenommen von Patienten mit Adipositas, keine besondere unterschiedliche Handhabung zu den Verlängerungen des Primärimplantates erforderte.

\subsubsection{Spondylodese, Endpunkt der Studie}

Zum Zeitpunkt des ausgefahrenen Sekundärimplantates waren die meisten Patienten soweit gewachsen, dass kein weiteres mitwachsendes Implantat nötig war und die Indikation zur Wirbelsäulenversteifung (Spondylodese) gestellt werden konnte. Zum gleichen Zeitpunkt wurden die Patienten des Vergleichskollektivs ohne operative Vorbehandlung (Kohorte 2a) bei bestehender neuromyopathischer Skoliose bei SMA der defini- 
tiven Operation zugeführt. Mit Ausnahme der Explantation, fand die Spondylodese der Patienten ohne operative Vorbehandlung (Kohorte 2a) in der gleichen Art und Weise wie bei der MAGEC®-Gruppe (Kohorte 1 und Kohorte 2b) statt.

Das Vorgehen bis zur endgültigen Versteifung bestand für die an SMA erkrankten Kinder mit mitwachsenden Implantaten aus zwei Schritten: Im ersten Schritt fand eine Operation zur Explantation des mitwachsenden Implantates statt, um eine bakterielle Besiedlung der Spondylodeseimplantate auszuschließen. Als zweiter Schritt erfolgte dann mit einem mehrwöchigen Abstand die dorsale Spondylodese. Das zweistufige Vorgehen wurde zur Vermeidung von Infektionen gewählt, da erkannt wurde, dass von 46 \% (Plaass et al. 2016) beziehungsweise $40 \%$ (Wagner et al. 2018) der Patienten Implantate bakteriell besiedelt waren.

Der Rahmen der stationären Aufnahme zur Explantationsoperation konnte auch für die Abklärungsdiagnostik zu der geplanten Spondylodese genutzt werden. Um den Patienten in der Zeit zwischen der Explantation und der Spondylodese das Sitzen zu erleichtern und eine übermäßige Zunahme der Wirbelsäulenkrümmung zu verhindern, wurde ein GipsKorsett individuell angepasst. Postoperativ wurde eine Kontrollröntgenuntersuchung durchgeführt. Eine Subgruppe von vier Patienten nach mitwachsenden Implantaten bekam zusätzlich zu den anterior posterior (a.p.) und seitlichen Röntgenaufnahmen auch Aufnahmen zur Prüfung der Aufdehnbarkeit der Skoliosekrümmung (Bendingaufnahmen). Die Kohorte 2a wurde in jedem Fall mit Bendingaufnahmen zusätzlich untersucht. Außerdem fanden Laboruntersuchungen, CT, MRT, pulmonologische und kardiologische Untersuchungen statt.

Das Operationsverfahren der Derotationsspondylodese (siehe Abb. 6) lässt sich in groben Zügen wie folgt beschreiben: Der Patient wurde in Bauchlage unter Allgemeinanästhesie operiert. Die Operation begann mit einer Längsinzision von etwa dem ersten Brustwirbelkörper bis etwa dem fünften Lendenwirbelkörper. Die thorakale Faszie wurde über den Dornfortsätzen inzidiert und die Rückenmuskulatur von den hinteren Wirbelbögen ab präpariert. Gemäß einer präoperativen Planung und der intraoperativen Navigation wurden die Pedikel von Brustwirbelkörper zwei bis Lendenwirbelkörper fünf mit einem Bohrer eröffnet und mit größenadaptierten Pedikalschrauben besetzt. Mittels eines Bildverstärkers wurde intraoperativ die korrekte Schraubenlage sichergestellt. Um eine Reposition zu ermöglichen wurden zum einen alle Muskel- beziehungsweise Sehnenansätze von den dorsalen Strukturen abgelöst und zum anderen in allen Etagen eine Hemiarthrektomie der Facettengelenke durchgeführt. Die eigentliche Reposition erfolgte durch das Einbringen von Titanstäben, die individuell auf den Patienten angepasst und an eine möglichst physiologische Kyphose und Lordose anmodelliert wurden. Das Einbringen von Spongiosachips und Knochenersatzstoffen diente zur Anregung einer dorsalen Fusion.

Bekam der Patient regelmäßig Nusinersen intrathekal appliziert oder war eine NusinersenTherapie (Spinraza $\left.{ }^{\circledR}\right)$ im Verlauf angedacht, wurde ein ca. $2 \times 3 \mathrm{~cm}$ großes Areal durch eine 
Hemilaminektomie geschaffen und bei der Fusion ausgespart, um eine Lumbalpunktion für die Medikamentenapplikation trotz der Spondylodese zu ermöglichen. Dessen Lokalisation wurde im Operationsbericht vermerkt und fotographisch dokumentiert (Lorenz et al. 2018). Vor der Entlassung wurde ein abschließendes sitzendes Röntgenbild in zwei Ebenen aufgenommen, um das Operationsergebnis beurteilen zu können. Diese Bilder wurden ebenfalls zur Analyse für diese Arbeit verwendet.

Die Spondylodese wurde als Endpunkt der Beobachtungszeit der Studie definiert. Alternativ galt der Dezember 2018 als Endpunkt der Beobachtungszeit und der Messungen für alle Patienten des Kollektivs, welche zu diesem Zeitpunkt noch keine Wirbelsäulenversteifung bekommen hatten, aber einen Nachuntersuchungszeitraum mit MAGEC®_ Implantaten von über vier Jahren.

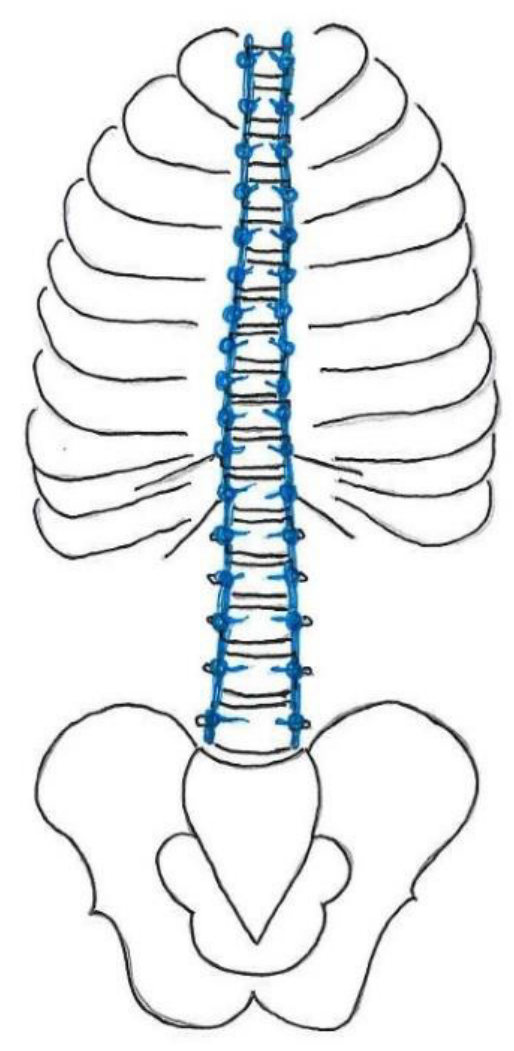

Abbildung 6: Skizze der versteiften Wirbelsäule nach der Spondylodese mit eingebrachten Pedikelschrauben von Th1 bis L5. 


\subsubsection{Nusinersen-Therapie (Spinraza $\left.{ }^{\circledR}\right)$}

Aufgrund des Studienerfolges von Nusinersen (Spinraza ${ }^{\circledR}$ ) (Finkel et al. 2017; Pechmann et al. 2019) und der darauffolgenden Zulassung des Medikamentes in Deutschland im Juni 2017, entschieden sich nach und nach auch Patienten dieses Studienkollektivs für die neue medikamentöse Therapie. Im Folgenden wird ein Überblick über das Therapieregime gegeben.

Zur Indikation für den Therapiebeginn mit Spinraza ${ }^{\circledR}$ sowie für den Antrag zur Kostenübernahme der Krankenkasse fand primär eine Genanalyse zur Bestimmung der Anzahl der SMN2-Kopien statt (Pechmann et al. 2019). Nach Bestätigung der SMN2-Kopien Anzahl und der Kostenübernahme startete die Aufdosierungsphase, bei der, jeweils im stationären Rahmen, an den Tagen 1, 14, 28, 63 und 180 Spinraza ${ }^{\circledR}$ in kurzer Allgemeinnarkose per Lumbalpunktion verabreicht wurde. Es schloss sich die Erhaltungstherapie an, welche aus intrathekalen Applikationen in einem Intervall von vier Monaten bestand.

\subsection{Datenerhebung}

Die Datenerhebung dieser Untersuchung war eine Fortführung der Datenerhebung von Batoul Badwan (Endpunkt war September 2016) (Lorenz et al. 2017). Von Dr. Heiko Lorenz (Oberarzt und Teil der Arbeitsgruppe Kinderorthopädie an der UMG) wurde eine neue Access Datenbank entwickelt, die der Datenverarbeitung und Analyse diente. Schließlich wurden die alten und die neu erhobenen Daten eingespeist und ausgewertet. Die im Folgenden beschriebenen Daten und Messungen wurden sowohl für das MAGEC®-Langzeitkollektiv (Kohorte 1) als auch für das Spondylodese Vergleichskollektiv (Kohorte 2) erhoben.

\subsubsection{Röntgenbildmessungen}

Die Röntgenbilder der gesamten Wirbelsäule in zwei Ebenen inklusive kompletter Abbildung der Beckenschaufeln wurden aufgrund fehlender Stehfähigkeit im Sitzen mittels anterior posteriorem (a.p.) und seitlichem Strahlengang aufgenommen. Die radiologischen Messungen an diesen Bildern wurden mit dem Programm Centricity Enterprise Web V3.0 (Version 3.0, 2006, General Electric Healthcare, Chalfont St. Giles in Buckinghamshire, Großbritannien) von zwei unabhängigen Untersuchern ausgemessen und gemittelt, um Messfehler und Abweichungsraten zu minimieren. 


\subsubsection{Wirbelsäulenkrümmungswinkel nach Cobb}

Im klinischen Alltag wird meistens der im Jahr 1948 von Cobb (Cobb 1948) beschriebene Wirbelsäulenkrümmungswinkel aufgrund seiner Einfachheit und hohen Praktikabilität verwandt. Das Verfahren wird allerdings ab und an kritisch hinterfragt, weil die dreidimensionale Deformität einer Skoliose nur in der Frontaleben erfasst wird. Zur Messung der Wirbelsäulenkrümmung nach Cobb wurden im anterior posterioren (a.p.) Strahlengang Tangenten an die Deckplatte des kranialen Wirbelkörpers der Krümmung (kranialer Neutralwirbelkörper, siehe N1 Abb. 7) sowie an die Bodenplatte des kaudalen Wirbelkörpers der Krümmung (kaudaler Neutralwirbelkörper, siehe N2 Abb. 7) gelegt und anschließend der dazwischenliegende Winkel bestimmt. Der von Scholten und Veldhuizen beschriebene Messfehler dieser Methode von fünf bis zehn Grad wird durch die Auswertung eines Mittelwertes von den Messungen zweier Untersucher verringert (Cobb 1948; Scholten und Veldhuizen 1987).

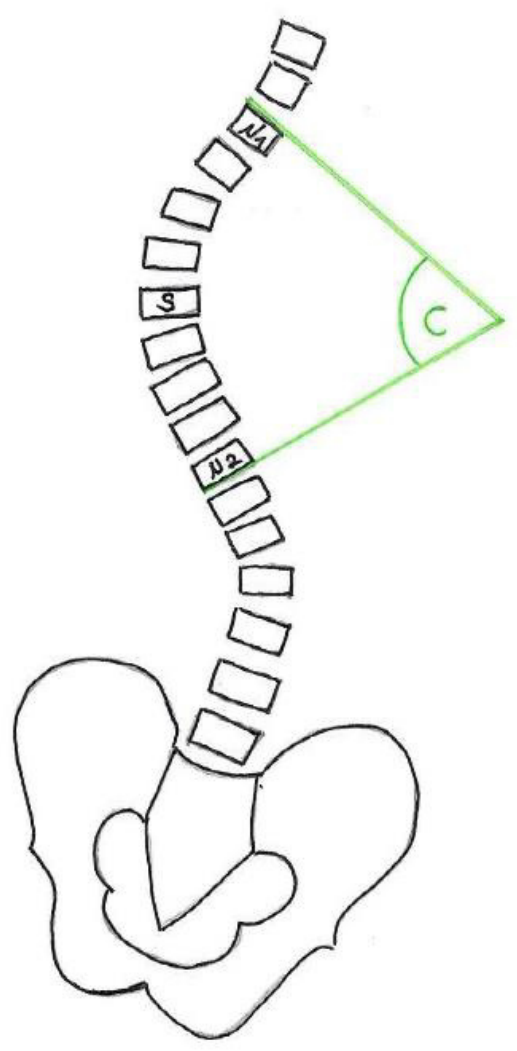

Abbildung 7: Skizze zur Messung des Cobb-Winkels. Er entspricht dem Winkel zwischen den Tangenten der Deckplatte des kranialen Neutralwirbels N1 und der Bodenplatte des kaudalen Neutralwirbels N2. S bezeichnet den Scheitelwirbel. 


\subsubsection{Beckenkippung}

Die Beckenkippung (Pelvic Obliquity) wurde als Winkel zwischen der Horizontalen und der Tangente entlang des Beckenkamms gemessen. Aufgrund von fehlender Steh- und Gehfähigkeit der Patienten konnte nicht wie für die Beckenkippung üblich ein im Stehen aufgenommenes Röntgenbild ausgemessen werden, sondern es musste auf ein im Sitzen aufgenommenes Bild zurückgegriffen werden. Für eine Kippung nach rechts (siehe Winkel P1 Abb. 8) wurde ein negatives Vorzeichen festgelegt und für eine Kippung nach links (siehe Winkel P2 Abb. 8) wurde ein positives Vorzeichen festgelegt.
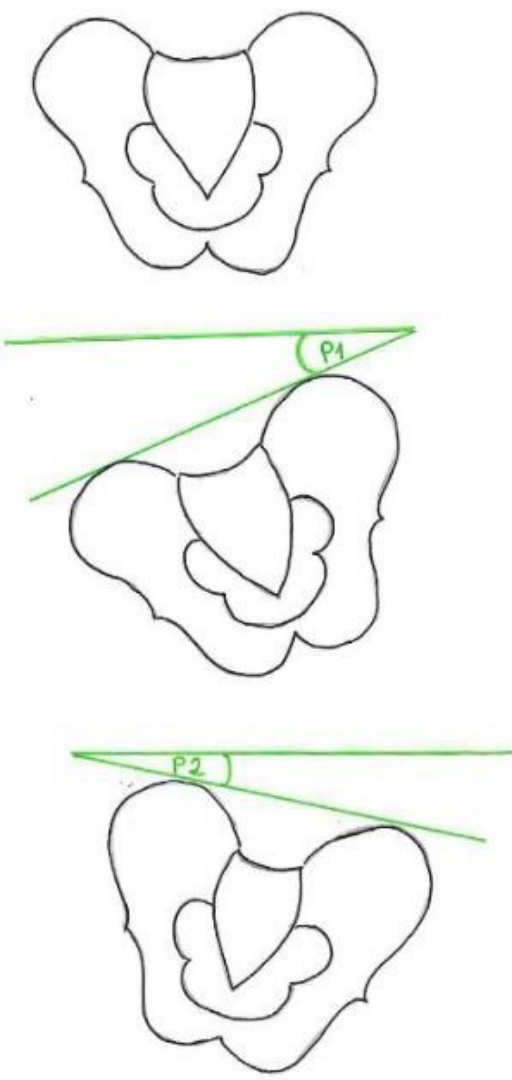

Abbildung 8: Skizze zur Messung des Beckenschiefstandes. Für eine Kippung nach rechts (P1) wurde ein negatives Vorzeichen festgelegt und für eine Kippung nach links (P2) wurde ein positives Vorzeichen gewählt. 


\subsubsection{Spinale Länge}

Die Spinale Länge (Spinal Length) (siehe Abb. 9) wurde im seitlichen Röntgenbild gemessen. Eine Waagerechte durch das Promontorium fungierte als kaudale Begrenzung der Spinalen Länge. Aufgrund von schlechter oder fehlender Kopfkontrolle wurde der kraniale Wirbelkörper nicht einheitlich bestimmt, sondern auf die Wirbelkörperhöhe der kranialen Instrumentierungsgrenze (Rippenhakenhöhe) festgelegt. Durch die individuelle Bestimmung des obersten Wirbelkörpers lassen sich die absoluten Werte der Spinalen Länge nicht vergleichen. Sie ist jedoch ein guter Verlaufsparameter und die individuelle beziehungsweise prozentuale Zunahme der Spinalen Länge ist im Vergleich auswertbar.

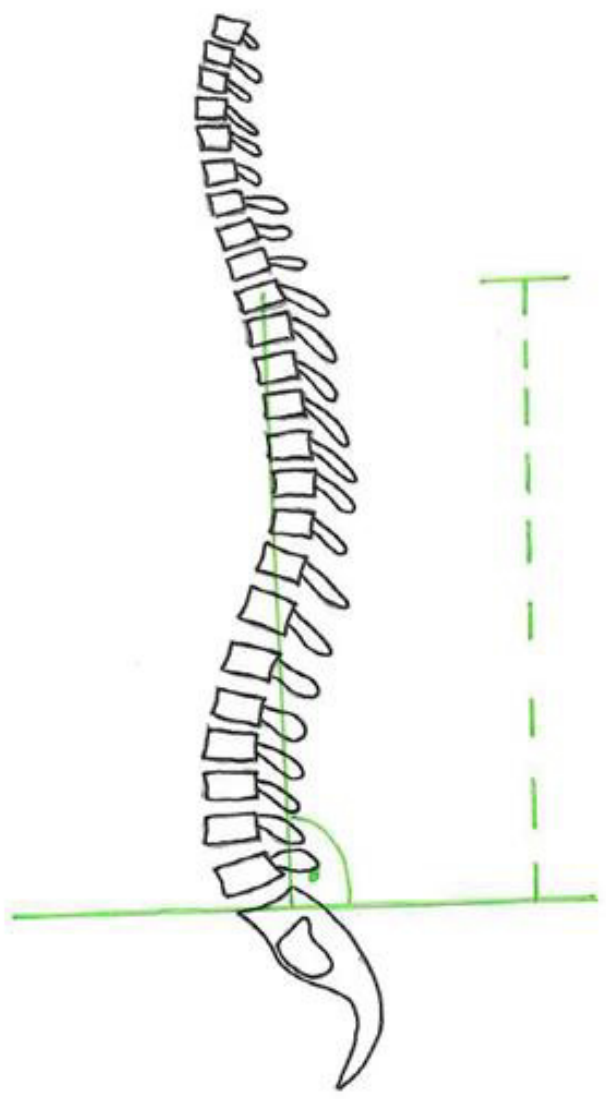

Abbildung 9: Skizze zur Messung der Spinalen Länge. Die untere Begrenzung bildet die Mitte der Oberkante des Promontoriums. Der Wirbelkörper der kranialen Messung ist abhängig von der Höhe der Instrumentierung. 


\subsubsection{Kyphose und Lordose}

Die Krümmungen der Brust- und Lendenwirbelsäule (siehe Abb. 10) wurden ebenfalls im seitlichen Bild gemessen. Die Kyphose wurde als Winkel zwischen der Tangente der Bodenplatte des zwölften Brustwirbelkörpers und der Tangente der Deckplatte des als obersten Wirbelkörper definierten Wirbelkörpers bestimmt. Hingegen wurde der Winkel zwischen der Tangente der Deckplatte des ersten Lendenwirbelkörpers und der Tangente der Bodenplatte des fünften Lendenwirbelkörpers zur Bestimmung der Lordose gemessen.

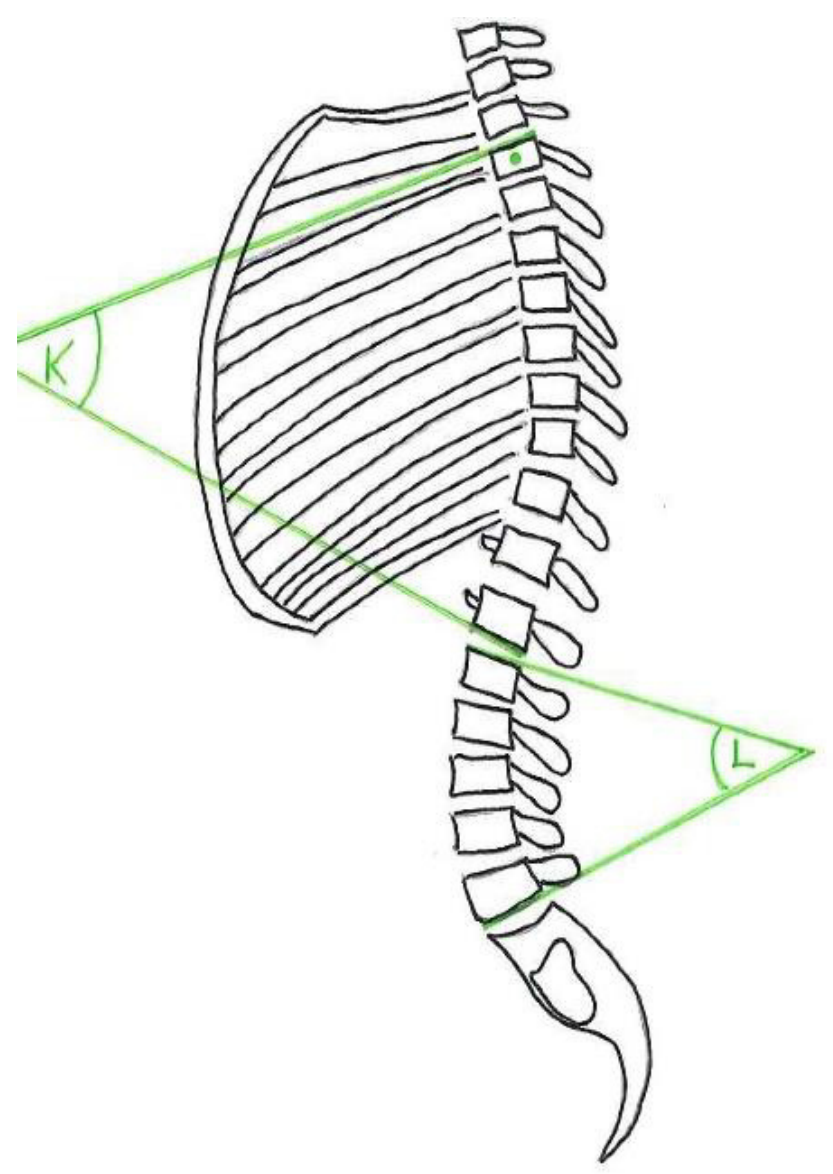

Abbildung 10: Skizze zur Messung der Kyphose und der Lordose. Winkel K bezeichnet die Kyphose zwischen der Deckplatte des am stärksten in die Krümmung geneigten Wirbelkörpers bis zur Bodenplatte Th12. Die Lordose ist durch den Winkel L beschrieben, welcher von der Deckplatte von L1 bis zur Bodenplatte von L5 reicht. 


\subsubsection{Erfassung weiterer Parameter}

Zusätzlich zu den Röntgenbildmessungen wurden Parameter wie Alter, Größe und Gewicht, Komplikationen und SMA spezifische Daten erfasst. Die Erhebung dieser Parameter erfolgte entweder im Rahmen der Patientenkontakte oder per Durchsicht der patientenbezogenen Arztbriefe in dem zur internen Zusammenarbeit und Archivierung genutzten Programm ixserv in der Version 4.25 (2010-2018 ixmid GmbH, Köln).

Zum Standard jeder Untersuchung gehörte die Messung und Dokumentation von Größe und Gewicht des Patienten. Das motorisch eingeschränkte Kollektiv dieser Studie machte eine Sitzwaage sowie eine Körperlängenmessung per Maßband im Sitzen notwendig. Wohingegen sich bei Säuglingen Perzentilenkurven zur Orientierung für Normalwerte am besten eignen, wurden in dieser Studie bei Schulkindern keine Perzentilen, sondern der Body-Mass-Index zur Einschätzung des Ernährungszustandes genutzt (Kromeyer-Hauschild et al. 2001).

\subsubsection{Komplikationen}

Für den Fall, dass Komplikationen aufgetreten waren, wurden diese in den Arztbriefen beschrieben und in der Datenbank vermerkt. Durch den Bezug dieser dokumentierten Komplikationen zur Gesamtanzahl von entsprechenden Interventionen, konnte eine Komplikationsrate ermittelt werden. Unterschieden wurde zwischen Implantat-bezogenen Komplikationen wie Ausbruch, Dislokation oder einem Nicht-Ausfahren des Implantates und operations-bezogenen Komplikationen wie Infekt, Wunddehiszenz oder Druckulkus. Dabei wurde beachtet, dass ein Knacken des MAGEC®-Implantates zunächst einmal nur das Erreichen der maximalen Spannung auf das Implantatgewinde aussagt, und damit ein Erreichen der in dem Moment maximalen Verlängerungsstrecke aufzeigte. Die Komplikation „Nicht-Ausfahren des Implantates“ meint die technische Insuffizienz beziehungsweise das totale Unvermögen der Verlängerung.

\subsection{Statistische Auswertung}

In Zusammenarbeit mit Dr. Heiko Lorenz (Oberarzt und Teil der Arbeitsgruppe der Kinderorthopädie an der UMG) entstand im Verlauf der Beobachtungszeit eine Datenbank, welche alle zuvor genannten Daten beinhaltet und mit dem Programm Access von Microsoft angelegt wurde. Die statistische Auswertung erfolgte durch Berechnung und Analyse der Mittelwerte der Messdaten, ihren Standardabweichungen und ihren Standardfehlern. Teils wurde dazu Access und teils Excel von Microsoft (Microsoft Corporation, One Microsoft Way, Redmond, WA 98052, USA) verwendet.

Das Signifikanzniveau wurde auf $\mathrm{p}<0,05\left(^{*}\right), \mathrm{p}<0,01\left(^{* *}\right)$ und $\mathrm{p}<0,001(* * *)$ festgelegt. 


\section{Ergebnisse}

Das untersuchte Patientenkollektiv für den MAGEC®-Langzeitverlauf (Kohorte 1) bestand aus 17 SMA-Patienten, davon acht Mädchen und neun Jungen, die in dem Zeitraum von 2011 bis 2018 mit MAGEC®-Implantaten behandelt und länger als vier Jahre beobachtet wurden. Dabei lag die mittlere Beobachtungszeit bei 4,6 Jahren. Neun von ihnen waren im Verlauf ausgewachsen und bekamen eine Spondylodese und zehn begannen im Beobachtungszeitraum eine Nusinersen-Therapie (Spinraza $\left.{ }^{\circledR}\right)$. Das durchschnittliche Alter bei der Primärimplatation lag bei 7,4 \pm 0,4 Jahren. Zwischen dem Beobachtungsbeginn und dem Abschluss lagen insgesamt 343 Distraktionen, davon 12-19 pro Patient. Nebenbefundlich wurde bei zwölf Patienten eine Hüftluxation festgestellt. Bei sechs Patienten war die Hüftluxation beidseits ausgeprägt.

Tabelle 1: Übersicht über die Charakteristika der Kohorte $1(n=17)$

\begin{tabular}{|l|l|}
\hline \multicolumn{2}{|c|}{ Kohorte 1 } \\
\hline Anzahl der Patienten & 17 SMA-Patienten (8 Mädchen, 9 Jungen) \\
\hline Verlaufsbeobachtungszeit & >4 Jahre beobachtet (im Mittel 4,6 Jahre lang) \\
\hline Alter bei Implantation & Im Mittel 7,4 Jahre alt bei Primärimplantation \\
\hline Spondylodesen & 9 Spondylodesen \\
\hline Nusinersen & Bei 12 Patienten (6 beidseits) \\
\hline Aüftluxationen & 10x Therapiebeginn mit Nusinersen \\
\hline
\end{tabular}




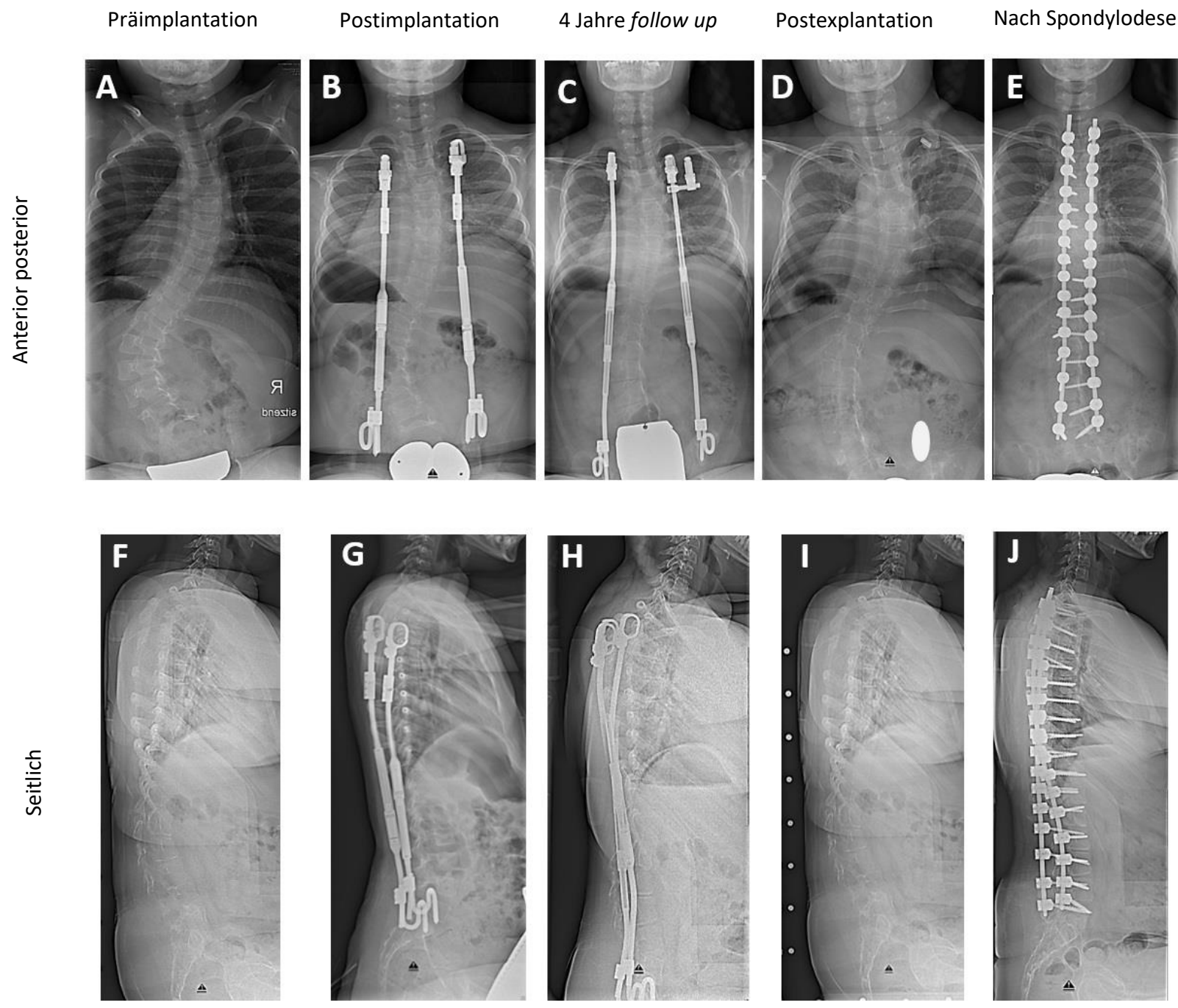

Abbildung 11: Dargestellt ist der Therapieverlauf der Kohorten 1 und 2b von Beginn bis Endpunkt der Beobachtungen am Beispiel der Röntgenbilder eines Patienten des Kollektivs. Untereinander finden sich jeweils die a.p. und die zugehörigen seitlichen Röntgenaufnahmen. Sie zeigen die Wirbelsäulendeformität vor Implantation, die Situation mit MAGEC®-Implantaten nach Implantation, den Zustand nach vier Jahren Beobachtungszeit mit ausgefahrenen MAGEC®-Implantaten, die Krümmung nach Explantation und den Abschluss der Behandlung nach Spondylodese (modifiziert nach Hell et al. 2020) 
In Kohorte 2, welche die Fragestellung 2 über das Outcome der SMA-Patienten mit oder ohne Vorbehandlung bei dorsaler Spondylodese beantworten sollte, wurden 28 SMAJugendliche eingeschlossen. 14 Patienten waren nicht vorbehandelt, davon sieben Mädchen und sieben Jungen. Weitere 14 Patienten, davon sechs Mädchen und acht Jungen, hatten während der Wachstumsphase mitwachsende Implantate erhalten, wobei acht Patienten

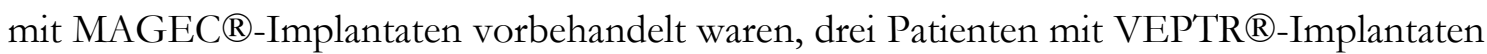

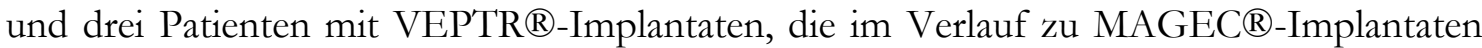
umgebaut wurden. Das mittlere Alter bei der Implantation betrug 8,6 (5,0-10,9) Jahre und das mittlere Alter bei Spondylodese 13,7 (9,7-17,0) Jahre bei den Patienten ohne Vorbehandlung beziehungsweise 13,8 (11,4-19,1) Jahre bei den Patienten mit Vorbehandlung.

Tabelle 2: Übersicht über die Charakteristika der Kohorte $2(n=28)$

\begin{tabular}{|c|c|}
\hline \multicolumn{2}{|l|}{ Kohorte 2a: } \\
\hline Anzahl der Patienten & $\begin{array}{l}14 \text { SMA-Patienten ohne Vorbehandlung } \\
\text { (7 Mädchen, } 7 \text { Jungen) }\end{array}$ \\
\hline Alter bei Spondylodese & Im Mittel 13,7 Jahre alt bei Spondylodese \\
\hline \multicolumn{2}{|l|}{ Kohorte 2b: } \\
\hline Anzahl der Patienten & $\begin{array}{l}14 \text { SMA-Patienten mit Vorbehandlung } \\
\text { (6 Mädchen, } 8 \text { Jungen) }\end{array}$ \\
\hline $\begin{array}{l}\text { Art des mitwachsenden } \\
\text { Implantates }\end{array}$ & 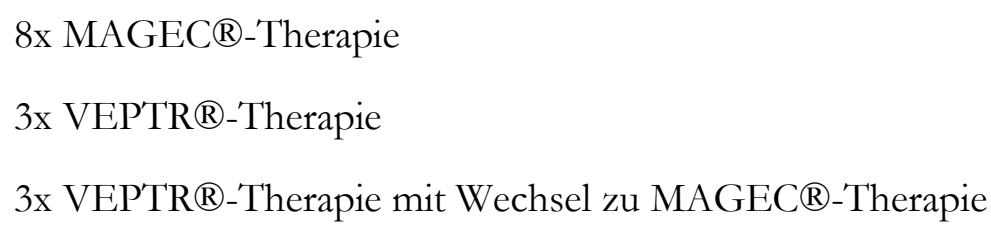 \\
\hline Alter bei Implantation & Im Mittel 8,5 Jahre alt bei Primärimplantation \\
\hline Alter bei Spondylodese & Im Mittel 13,8 Jahre alt bei Spondylodese \\
\hline
\end{tabular}




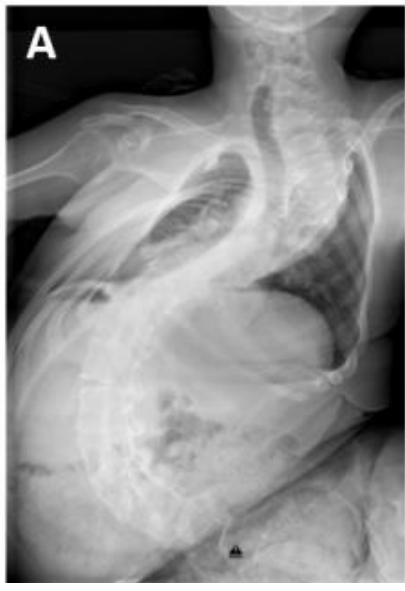

Vor Spondylodese

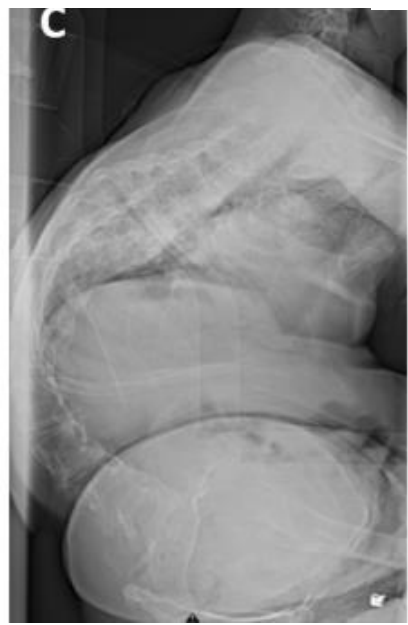

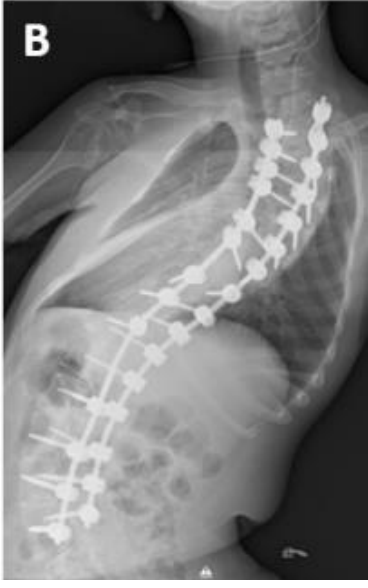

Nach Spondylodese

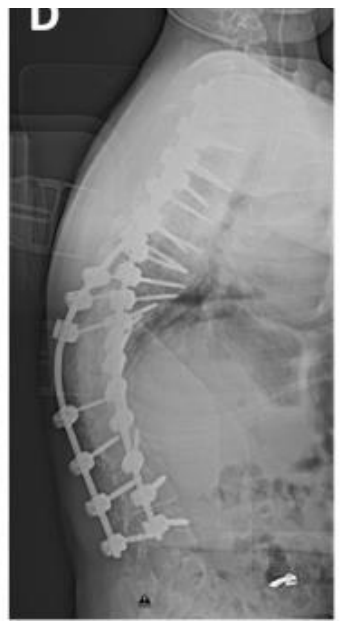

Abbildung 12: Dargestellt sind Röntgenbilder eines Patienten der Kohorte 2a. Die paarweise untereinander stehenden Aufnahmen zeigen die Skoliosekrümmung in a.p. und in seitlichem Strahlengang zu den Zeitpunkten vor Spondylodese und nach Spondylodese (modifiziert nach Hell et al. 2020).

\subsection{Radiologische Auswertung von Kohorte 1}

Die radiologischen Daten von Kohorte $1(n=17)$ wurde in Bezug auf die Fragestellungen, wie sich die Wirbelsäulenkrümmung bei SMA-Kindern, die mit bilateralen MAGEC®Implantaten versorgt wurden, im Langzeitverlauf verhält und ob ein law of diminishing returns beobachtet werden kann, ausgewertet. 


\subsubsection{Krümmungswinkel nach Cobb}

Bei allen SMA-Kindern konnte die Skoliosekrümmung von durchschnittlich $70^{\circ}\left( \pm 6^{\circ}\right)$ auf $29^{\circ}\left( \pm 4^{\circ}\right)$ durch die primäre MAGEC®-Implantation signifikant $(\mathrm{p}<0,001)$ verbessert werden, was einer Korrektur von 59 \% entspricht (siehe Abb. 13). Von Verlängerung 1 $(\mathrm{n}=17)$ bis Verlängerung $16(\mathrm{n}=9)$ lag der Skoliosekrümmungswinkel konstant um $35^{\circ}( \pm$ $2^{\circ}$ ) (siehe blaue Trendlinie Abb. 13). Der Zeitpunkt Verlängerung 10 entsprach dabei etwa vier Jahren Beobachtungszeit.

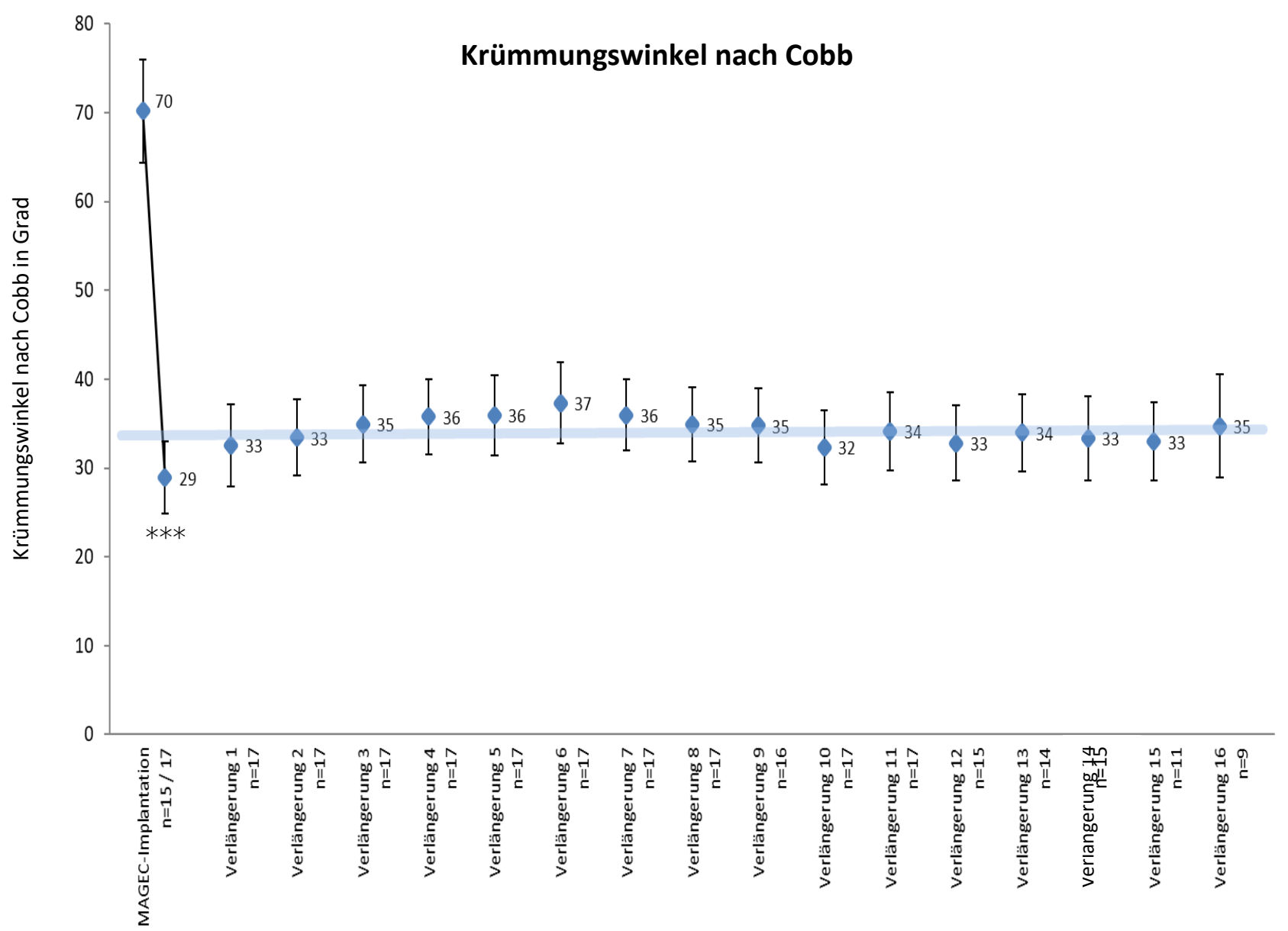

Abbildung 13: Ergebnisse der Messungen des Krümmungswinkels nach Cobb in der Kohorte 1 (n=17 Patienten) im Zeitverlauf der Verlängerungen. Dargestellt sind die Mittelwerte der Winkel in Grad und ihr Standardfehler. Die Veränderung durch die Primäroperation von im Mittel $70^{\circ}$ auf im Mittel $29^{\circ}$ war signifikant $(\mathrm{p}<0,001(* * *))$. 


\subsubsection{Beckenschiefstand}

Der Beckenschiefstand änderte sich mit der Primärimplantation von $17,7^{\circ}\left( \pm 11,3^{\circ}\right)$ auf $4,5^{\circ}\left( \pm 2,9^{\circ}\right)$ (siehe Abb.14). Die Abnahme des Beckenschiefstandes war nicht signifikant $(p=0,16)$. Im Verlauf der regelmäßigen Verlängerungen blieb er konstant bei $4,6^{\circ}\left( \pm 0,3^{\circ}\right)$.

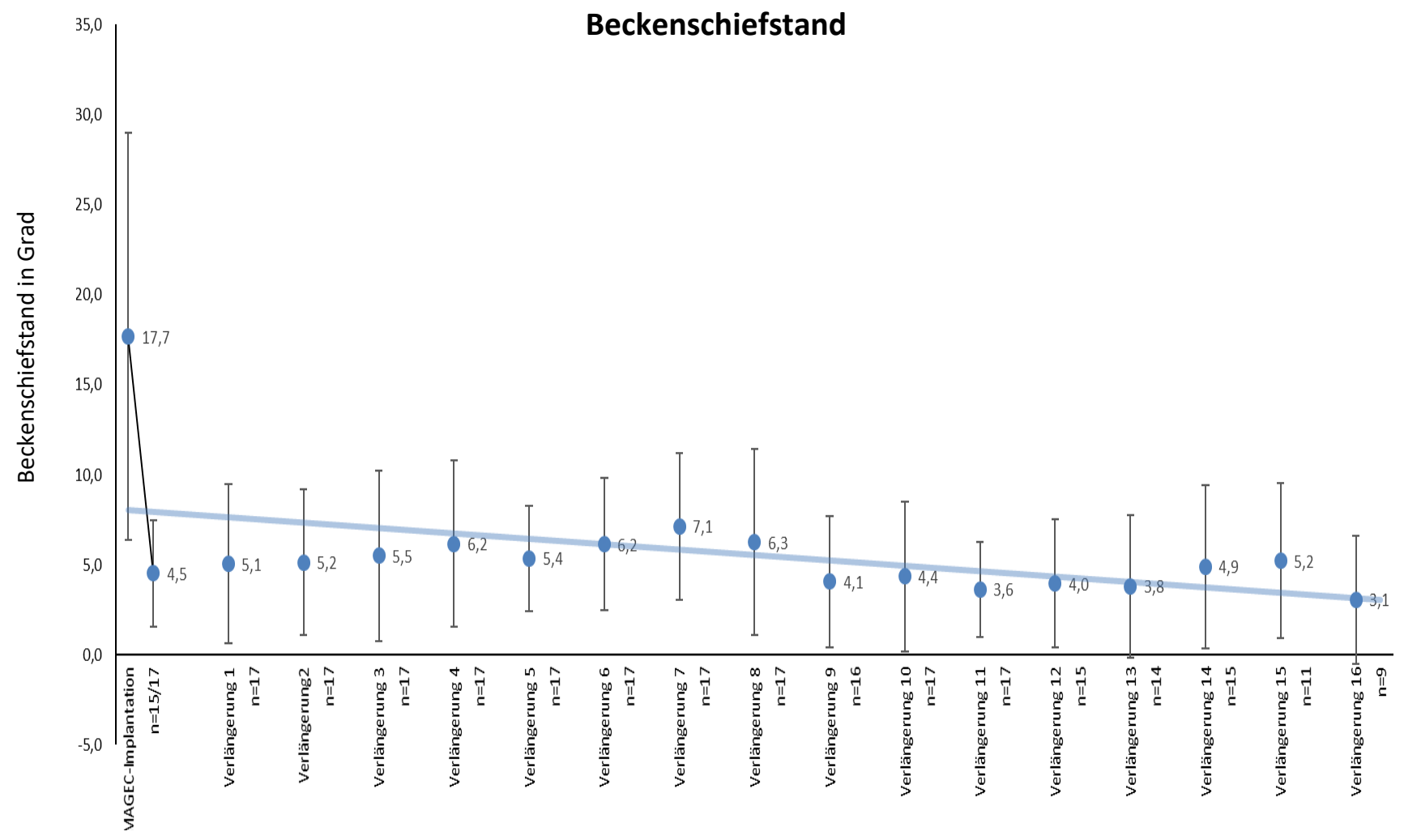

Abbildung 14: Ergebnisse der Messungen des Beckenschiefstandes in der Kohorte 1 im Zeitverlauf der Verlängerungen. Dargestellt sind die Mittelwerte in Grad und ihre Standardabweichungen. Die Veränderung durch die Primärimplantation von im Mittel 17,7 auf 4,5 war nicht signifikant $(\mathrm{p}=0,16)$. 


\subsubsection{Spinale Länge}

Durch die Primärimplantation konnte die Spinale Länge (siehe Abb. 15) im Gesamtkollektiv von durchschnittlich $236 \mathrm{~mm}$ ( $\pm 24 \mathrm{~mm}$ ) auf $289 \mathrm{~mm}$ ( $\pm 19 \mathrm{~mm}$ ) erhöht werden, was einer signifikanten $(\mathrm{p}<0,001)$ Zunahme von $53 \mathrm{~mm}$ entsprach. Von Verlängerung 1 ( $n=17)$ bis Verlängerung $16(n=9)$ nahmen erwartete Spinale Länge und reelle Spinale Länge kontinuierlich zu. Die erwartete Spinale Länge lag bei $+5 \mathrm{~mm}$ pro Verlängerung (siehe grüne Trendlinie Abb.15). Die reelle Spinale Länge wuchs $+4,3 \mathrm{~mm}$ pro Verlängerung (siehe rote Trendlinie Abb.15). Die Gegenüberstellung der erwarteten zur reellen Zunahme der Spinalen Länge ist in der Dissertation von Jasmin Grote aufgeführt (Lorenz et al. 2019). Der Zeitpunkt Verlängerung 10 entsprach etwa vier Jahren Beobachtungszeit.

\section{Spinale Länge}

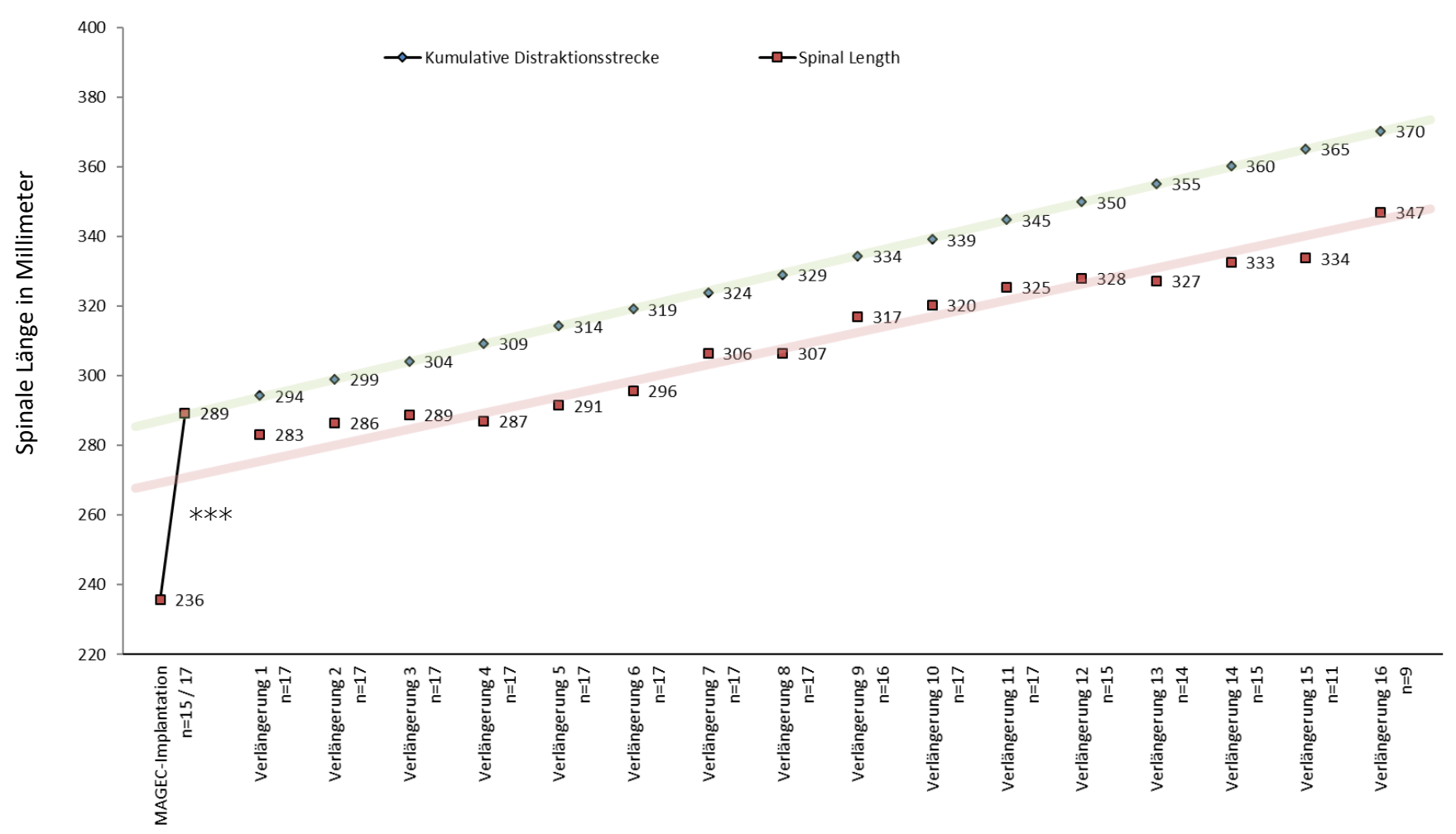

Abbildung 15: Darstellung der Spinalen Länge der Kohorte 1 ( $n=17$ Patienten) im Zeitverlauf. Es wird die erwartete Spinale Länge (grün) der reellen Spinalen Länge (rot) gegenübergestellt. Dargestellt sind durchschnittliche absolute Werte in Millimetern im Zeitverlauf der Verlängerungen. Die Spinale Länge nahm stetig zu. Bei einer erwarteten Zunahme von $+5 \mathrm{~mm}$ pro Verlängerung (grüne Trendlinie) ergab sich ein tatsächlicher Zuwachs von im Mittel $+4,3 \mathrm{~mm}$ pro Verlängerung (rote Trendlinie). Die Verlängerungen fanden postoperativ im Abstand von fünf Monaten zur Implantation und im weiteren Verlauf alle drei Monate statt. Die Veränderung durch die Primärimplantation von im Mittel $236 \mathrm{~mm}$ auf im Mittel $289 \mathrm{~mm}$ war signifikant $(\mathrm{p}<0,001(* * *))$. 


\subsubsection{Kyphose und Lordose}

Nach einer primären signifikanten Reduktion der Kyphose durch die MAGEC ${ }^{\circledR}$ Implantation von $44,5^{\circ}$ auf $34,3^{\circ}(p<0,01)$ und einer leichten Erhöhung der Lordosewerte von $28,4^{\circ}$ auf $30,8^{\circ}(p=0,23)$ undulierten die Kyphose- und Lordosewerte (siehe Abb.16) während der gesamten Verlängerungsphase im physiologischen Bereich (Giglio und Volpon 2007). Der Kyphosewinkel lag um $37^{\circ}$ (siehe grüne Trendlinie Abb.16) und der Lordosewinkel um $25^{\circ}$ (siehe rote Trendlinie Abb.16) im Durchschnitt. Der Zeitpunkt Verlängerung 10 entsprach etwa vier Jahren Beobachtungszeit.

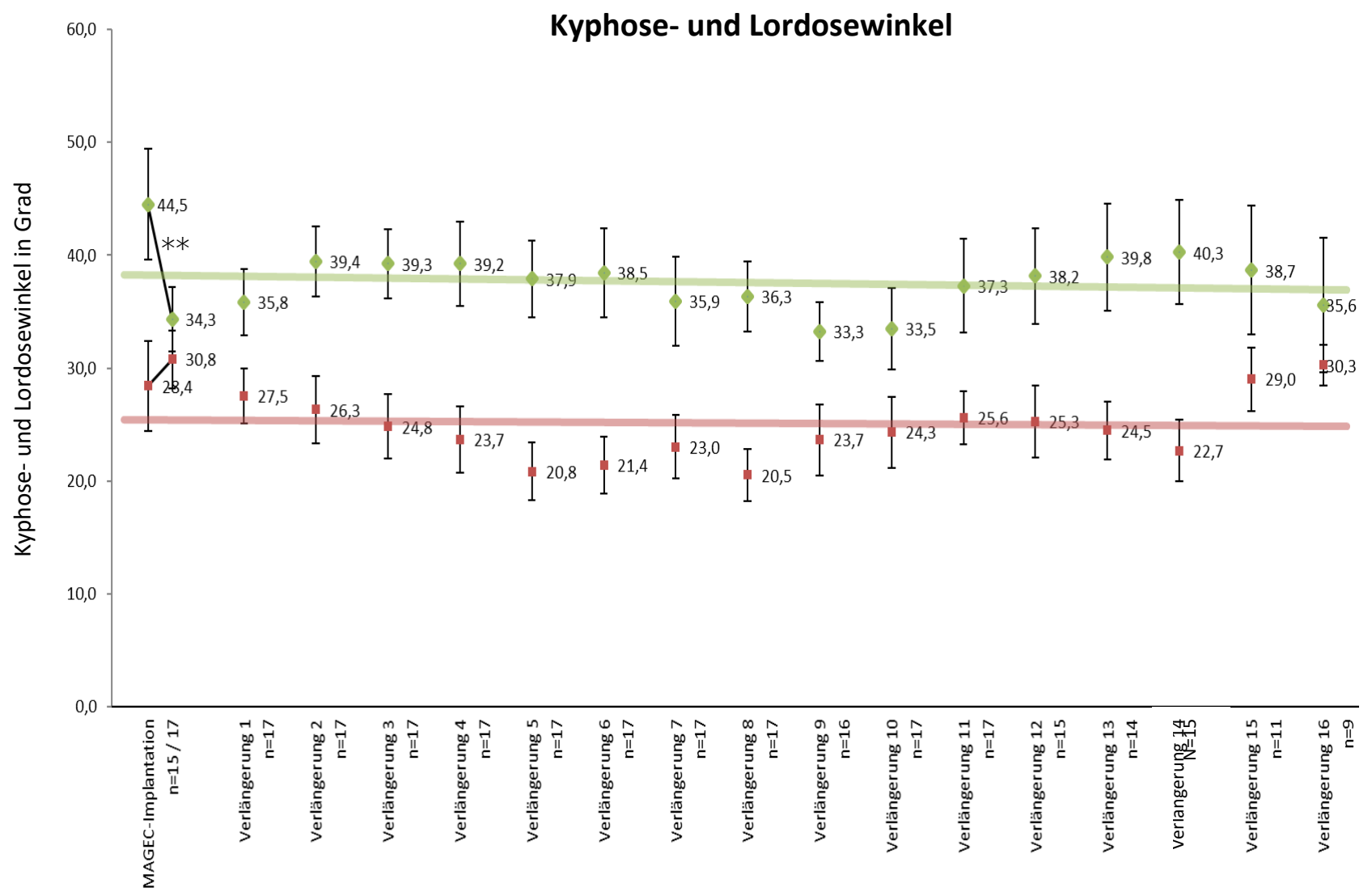

Abbildung 16: Kyphose- und Lordosewinkel der Kohorte 1 ( $n=17$ Patienten) im Zeitverlauf. Dargestellt sind Mittelwerte der Winkel in Grad und ihre Standardfehler. Der Kyphosewinkel (grün) lag über den kompletten Verlängerungszeitraum im Mittel um $37^{\circ}$ (grüne Trendlinie). Der Lordosewinkel (rot) lag über den kompletten Verlängerungszeitraum im Mittel um $25^{\circ}$ (rote Trendlinie). Die Veränderung des Kyphosewinkels bei der Primäroperation war signifikant $(\mathrm{p}<0,01(* *))$. Die Veränderung des Lordosewinkels bei der Primäroperation war nicht signifikant $(p=0,23)$. 


\subsection{Radiologische Auswertung von Kohorte 2}

Die radiologischen Daten von Kohorte $2(n=28)$ wurde in Bezug auf die Fragestellung, ob SMA-Kinder, welche mit MAGEC®- oder VEPTR®-Implantaten über Jahre behandelt wurden bessere Ergebnisse nach der endgültigen Spondylodese haben als unbehandelte SMA-Jugendliche, ausgewertet.

\subsubsection{Krümmungswinkel nach Cobb}

Die Auswertung des Cobb-Winkels (siehe Abb. 17) war für die Kohorte 2b ( $n=14)$ in fünf Untersuchungen während ihres Therapieverlaufs gegliedert. Dazu gehören: Die Primärimplantation mit Auswertung der Röntgenbilder vor und nach der MAGEC®- oder VEPTR®-Primäroperation, die letzte Untersuchung mit Röntgenbild mit liegenden MAGEC®- oder VEPTR ${ }^{\circledR}-S y s t e m$, der Ausbau des mitwachsenden Implantates mit Auswertung des postoperativen Bildes sowie die Spondylodese und die dazugehörigen präund postoperativen Röntgenbilder. Und zusätzlich eine Untersuchung bei vier Jugendlichen der Kohorte 2b, bei denen nach dem Ausbau der MAGEC®-Implantate oder VEPTR ${ }^{\circledR}$-Implantate Bendingaufnahmen erstellt wurden.

Für die Kohorte 2a wurden die Röntgenbilder vor und nach Spondylodese, sowie Bendingaufnahmen von allen Patienten $(n=14)$ ausgewertet.

Erwartungsgemäß konnte in der Kohorte $2 \mathrm{~b}$ durch die Primärimplantation von MAGEC®-Implantaten oder VEPTR ${ }^{\circledR}$-Implantaten im durchschnittlichen Alter von 8,6 Jahren eine signifikante $(\mathrm{p}<0,001)$ Krümmungskorrektur um $53 \%$ von $69^{\circ}$ auf $32^{\circ}$ erreicht werden. Nach einem durchschnittlichen Follow-up von 4,9 Jahren mit regelmäßigen Verlängerungen fand sich ein Skoliosekrümmungswinkel von durchschnittlich $44^{\circ}$. Dieser Wert war im Vergleich zum unmittelbar postoperativen Wert, 4,9 Jahre vorher, nicht signifikant verändert $(\mathrm{p}=0,087)$. Nach dem Ausbau der MAGEC®-Implantate oder der VEPTR ${ }^{\circledR}$-Implantate verschlechterte sich die Krümmung erneut auf durchschnittlich $71^{\circ}$, was dem Ausgangswert bei Therapiebeginn entspricht. Die Analyse der Bendingaufnahmen von vier Patienten dieser Kohorte ergab eine Krümmungsreduktion auf $53^{\circ}$, was eine Verbesserung von $25 \%$ erzielte. Schließlich konnte durch die Spondylodese eine signifikante $(\mathrm{p}<0,001)$ Krümmungsverbesserung um $54 \%$ von $71^{\circ}$ auf $33^{\circ}$ erreicht werden.

Bei den SMA-Patienten ohne Vorbehandlung (Kohorte 2a) zeigte sich durchschnittlich ein präoperativer Krümmungswinkel von $104^{\circ}$ (siehe Abb. 17). Der Unterschied dieser Krümmung in der Kohorte 2a zum Skoliosekrümmungswinkel vor Spondylodese in der Kohorte $2 \mathrm{~b}\left(71^{\circ}\right)$ war signifikant $(\mathrm{p}=0,005)$. In den präoperativen Bendingaufnahmen $(\mathrm{n}=14)$ von Kohorte $2 \mathrm{a}$ konnte eine Aufdehnung von $104^{\circ}$ auf $66^{\circ}$ erreicht werden, was einer Korrektur von $37 \%$ entsprach. Die anschließende Spondylodese korrigiert den Krümmungswinkel in Kohorte 2 a signifikant $(\mathrm{p}<0,001)$ um $52 \%$ von $104^{\circ}$ auf $50^{\circ}$. Die Ergebnisse des Skoliosekrümmungswinkels von Kohorte 2a $\left(50^{\circ}\right)$ im Vergleich zu der Kohorte $2 \mathrm{~b}\left(33^{\circ}\right)$ waren signifikant schlechter $(\mathrm{p}=0,037)$. 


\section{Krümmungswinkel nach Cobb}

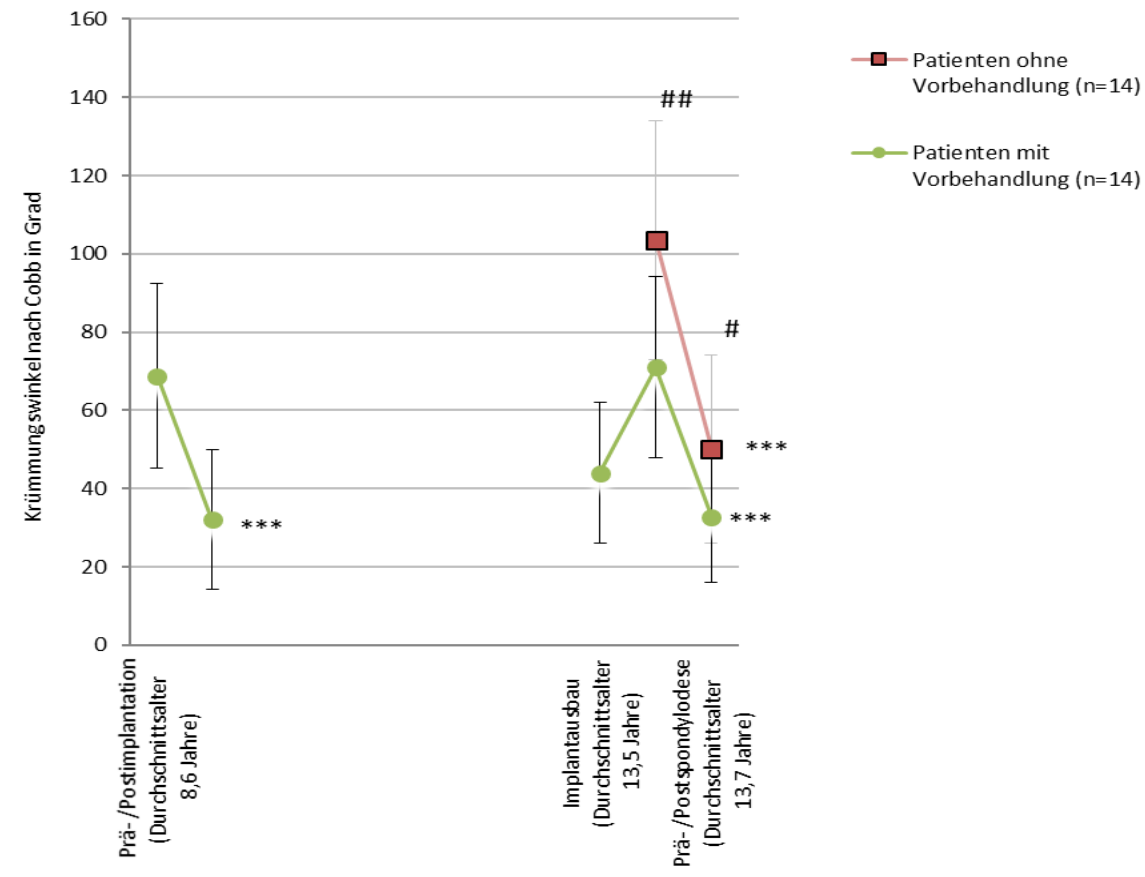

Abbildung 17: Darstellung des Skoliosekrümmungswinkels in Grad von der Kohorte 2b (n=14), das heißt von SMA-Kindern mit einer MAGEC $®$ - oder VEPTR ${ }^{\circledR}-B e h a n d l u n g$ für durchschnittlich 4,9 Jahre vor Spondylodese (grün) im Vergleich zu der Kohorte 2a (n=14) (rot), die nicht operativ vorbehandelt war. Eingezeichnet sind die Untersuchungszeitpunkte Implantation, Ausbau und Spondylodese. ${ }^{* * *} \mathrm{p}<0,001$ (innerhalb eines Kollektivs, bezogen auf den vorherigen Wert) \# p<0,05 und \#\# $\mathrm{p}<0,01$ (zwischen den beiden Gruppen, innerhalb eines Zeitpunktes) (modifiziert nach Hell et al. 2020).

\subsubsection{Beckenschiefstand}

Die Auswertung des Beckenschiefstandes (siehe Abb. 18) wurde für die Kohorten 2a und $2 \mathrm{~b} z u$ den identischen Zeitpunkten wie für die Skoliosekrümmung (siehe 3.2.1) sowie für die weiteren radiologischen Messungen vorgenommen.

In der Kohorte $2 \mathrm{~b}$ erzielte die Primärimplantation von bilateralen MAGEC®-Implantaten oder VEPTR®-Implantaten eine signifikante $(\mathrm{p}<0,001)$ Beckenschiefstandsänderung von $15,75^{\circ}$ auf $4,56^{\circ}$. Dieser Wert konnte über die Jahre gehalten werden, sodass nach 4,9 Jahren Therapieverlauf der Beckenschiefstand vor Explantation der Implantate bei durchschnittlich $3,79^{\circ}$ lag. Es ergab sich hier kein signifikanter Unterschied $(p=0,751)$. Nach Explantation der MAGEC $®$-Implantate oder der VEPTR®-Implantate

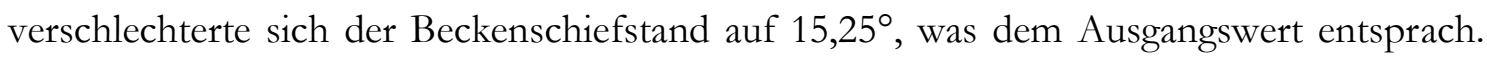
Durch die Spondylodese konnte erneut eine signifikante $(p=0,016)$ Reduktion auf 5,68 erreicht werden. 
In der Kohorte 2a bewirkte die Spondylodese eine signifikante $(\mathrm{p}=0,019)$ Verminderung des Beckenschiefstandes von $20,95^{\circ}$ auf $12,4^{\circ}$.

Somit zeigte sich letztendlich in der Kohorte $2 \mathrm{~b}$ als abschließendes Ergebnis ein geringerer Beckenschiefstand als in der Kohorte 2a (5,68 versus $\left.12,4^{\circ}\right)$, wobei dieser Unterschied nicht signifikant war $(\mathrm{p}=0,078)$.

\section{Beckenschiefstand}

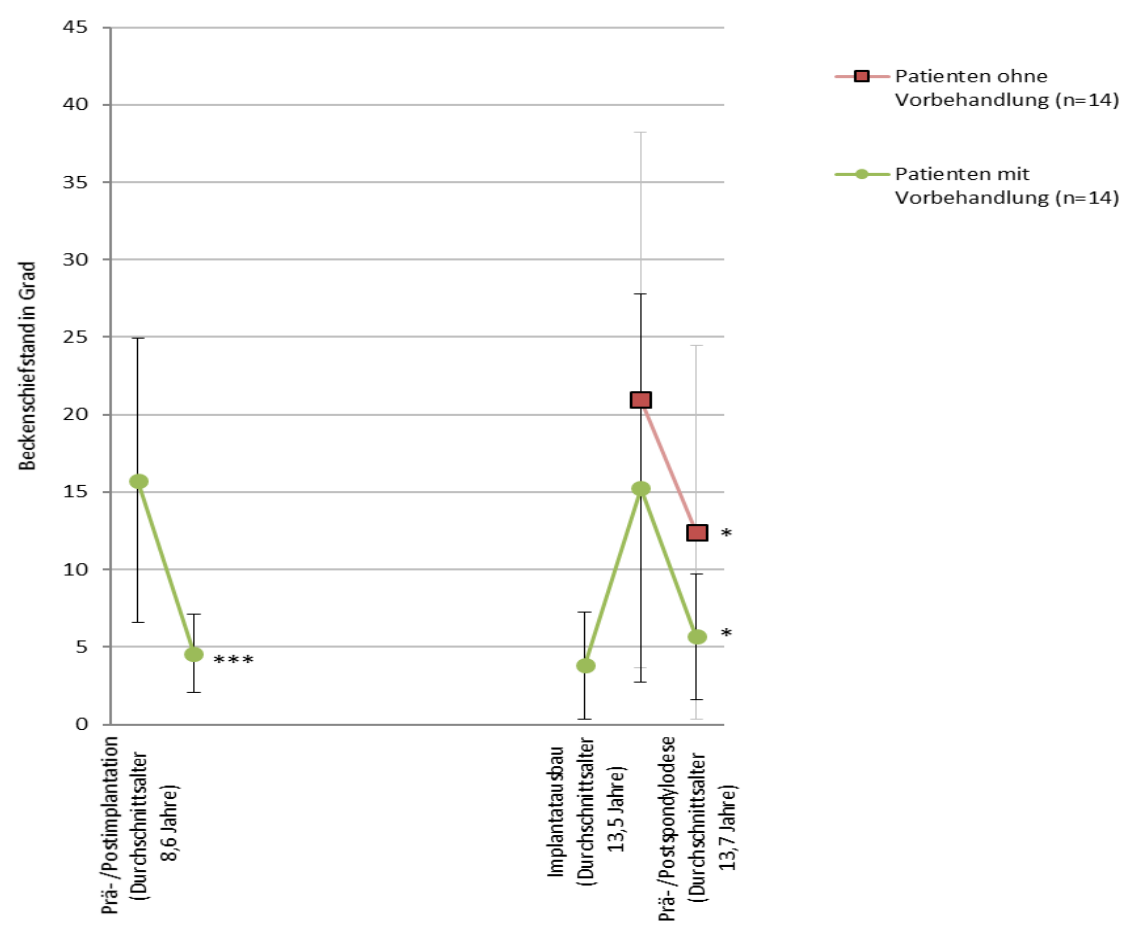

Abbildung 18: Darstellung des Beckenschiefstandes in Grad von der Kohorte 2b ( $\mathrm{n}=14)$, das heißt von SMA-Kindern mit einer MAGEC®- oder VEPTR®-Behandlung für durchschnittlich 4,9 Jahre vor Spondylodese (grün) im Vergleich zu der Kohorte 2a $(n=14)$ (rot), die nicht operative vorbehandelt war. Eingezeichnet sind die Untersuchungszeitpunkte Implantation, Ausbau und Spondylodese. ${ }^{*} \mathrm{p}<0,05$ und ${ }^{* * *} \mathrm{p}<0,001$ (innerhalb eines Kollektivs, bezogen auf den vorherigen Wert) (modifiziert nach Hell et al. 2020).

\subsubsection{Spinale Länge}

Die Auswertung der Spinalen Länge (siehe Abb. 19) erfolgte zu den oben beschriebenen Zeitpunkten (siehe 3.2.1).

Bei der Kohorte 2b erzielte die Primärimplantation eine signifikante $(\mathrm{p}<0,001)$ durchschnittliche Zunahme der Spinalen Länge von $246 \mathrm{~mm}$ auf $294 \mathrm{~mm}$ (um $48 \mathrm{~mm}$ ). Dies entsprach einer Verlängerung der Wirbelsäule um $20 \%$. Im Verlauf der Behandlung stieg die Spinale Länge erwartungsgemäß auf durchschnittlich $329 \mathrm{~mm}$ an. Nach Explantation der MAGEC®-Implantate oder der VEPTR®-Implantate verringerte sich die Spinale 
Länge auf $293 \mathrm{~mm}$, was über dem primären Wert von $246 \mathrm{~mm}$ liegt. Die Spondylodese bewirkte erneut eine signifikante ( $p<0,001)$ Längenzunahme von $293 \mathrm{~mm}$ auf $340 \mathrm{~mm}$ im Durchschnitt, was einem absoluten Wert von $47 \mathrm{~mm}$ und einer prozentualen Zunahme von $16 \%$ entsprach.

Bei der Kohorte 2a bewirkt die Spondylodese eine signifikante $(\mathrm{p}<0,001)$ Vergrößerung der Spinalen Länge von $280 \mathrm{~mm}$ auf $348 \mathrm{~mm}$, was einen absoluten Wert von $68 \mathrm{~mm}$ und eine prozentuale Zunahme von $24 \%$ ergab.

Letztendlich zeigte sich als Endsituation bei identischem Alter eine vergleichbare Spinale Länge nach Spondylodese von $340 \mathrm{~mm}$ der Kohorte $2 \mathrm{~b}$ gegenüber $348 \mathrm{~mm}$ der Kohorte 2a. Dieser Unterschied war nicht signifikant $(\mathrm{p}=0,564)$.

\section{Spinale Länge}

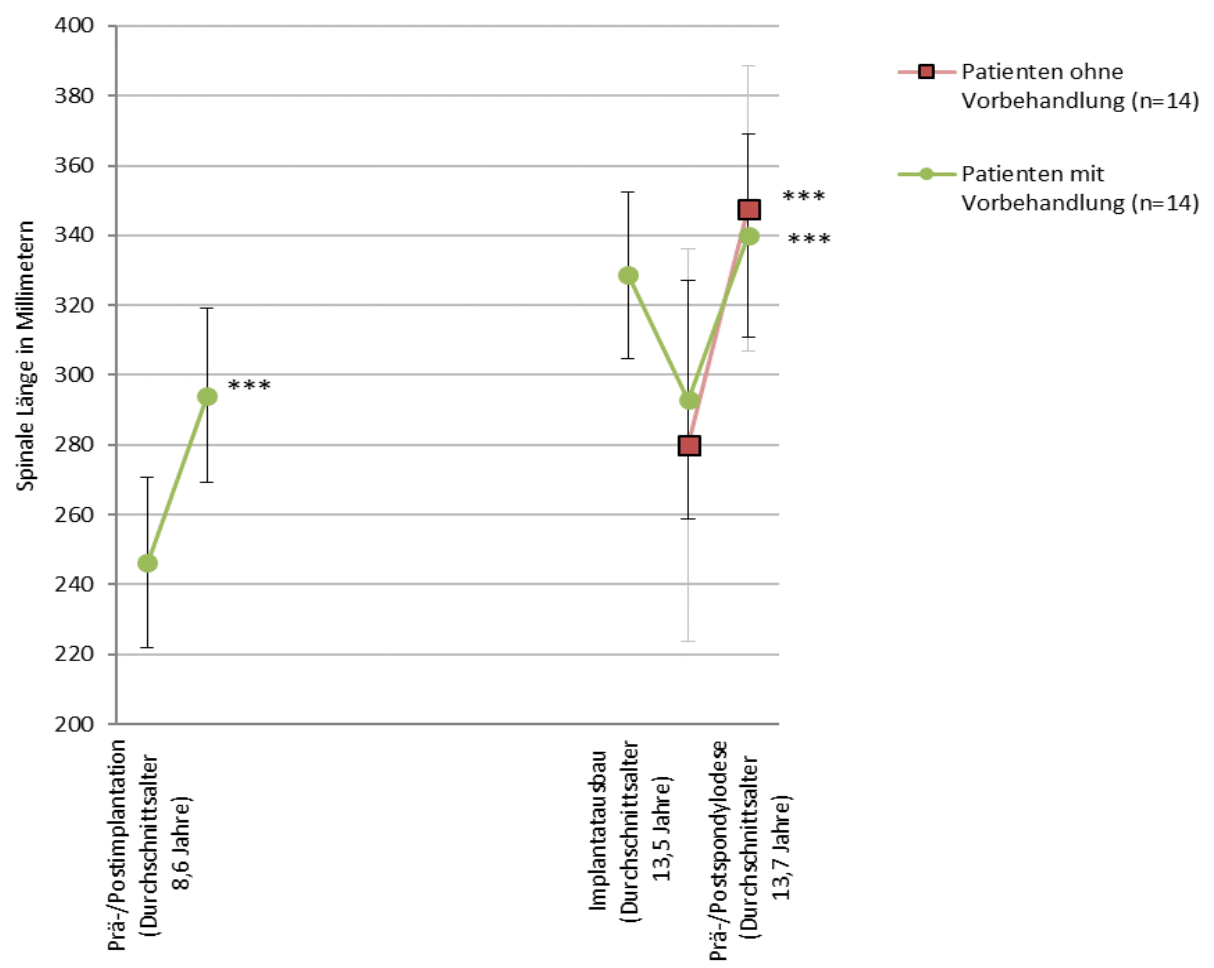

Abbildung 19: Darstellung der Spinalen Länge in Millimetern von der Kohorte 2b ( $=14)$, das heißt von SMA-Kindern mit einer MAGEC ${ }^{\circledR}$ - oder VEPTR ${ }^{\circledR}-B e h a n d l u n g$ für durchschnittlich 4,9 Jahre vor Spondylodese (grün) im Vergleich zu der Kohorte 2a (n=14) (rot), die keinerlei operative Vorbehandlung hatten. Eingezeichnet sind die Untersuchungszeitpunkte Implantation, Ausbau und Spondylodese. ${ }^{* * *} \mathrm{p}<0,001$ (innerhalb eines Kollektivs, bezogen auf den vorherigen Wert) (modifiziert nach Hell et al. 2020). 


\subsubsection{Kyphose und Lordose}

Die Primärimplantation bei den Patienten mit Vorbehandlung durch ein mitwachsendes Implantat (Kohorte $2 b)$ änderte den thorakalen Kyphosewinkel signifikant $(p=0,041)$ von $42^{\circ}$ auf $33^{\circ}$. Das entspricht einer Verringerung um $22 \%$. Der lumbale Lordosewinkel wurde von $35^{\circ}$ auf $33^{\circ}$ korrigiert $(\mathrm{p}=0,470)$. Die Spondylodese derselben Gruppe änderte den Kyphosewinkel von $46^{\circ}$ auf $32^{\circ}(\mathrm{p}=0,059)$ und den Lordosewinkel von $30^{\circ}$ auf $31^{\circ}$ $(\mathrm{p}=0,816)$.

Die Spondylodese bei den Patienten ohne Vorbehandlung (Kohorte 2a) ergab einen präoperativen thorakalen Kyphosewinkel von $44^{\circ}$ zu postoperativ $38^{\circ}(\mathrm{p}=0,304)$ und einen präoperativen lumbalen Lordosewinkel von $43^{\circ}$ zu postoperativ $40^{\circ}(\mathrm{p}=0,234)$.

\subsection{Auswertung der Patientenaktenrecherche}

\subsubsection{Komplikationen}

Die Auswertung der Komplikationen teilte sich in die mit der Operation assoziierten Komplikationen und die Komplikationen der konservativen Therapie.

Operativ bedingt traten zweimal Druckulcera bei kachektischen Patienten auf, zweimal Infektionen im Sinne von Keimbesiedlung der Implantate nach Ulcera und vier Revisionsoperationen aufgrund eines diskonnektierten VEPTR®-Rippenhakens und dreier dislozierter VEPTR®-Rippenhaken. Somit lag hier die Komplikationsrate von operativ assoziierten Komplikationen bezogen auf die Anzahl der untersuchten Personen ( $\mathrm{n}=17)$ bei $47 \%$ und bezogen auf die Anzahl der durchgeführten Operationen (52, 2-4 pro Patient) bei $15 \%$.

Im konservativen Therapieverlauf trat eine Wunddehiszenz auf, sowie ein nicht ausfahrbares Implantat wegen zu großer Spannung (bei einem adipösen Patienten mit $>45 \mathrm{~kg}$ ), was zweimal eine operative Distraktion notwendig machte. Außerdem erfolgten eine Notfallvorstellung bei Lumbago und ein Implantatversagen, bei dem nach übermäßiger Distraktion die Implantatstangen diskonnektierten. Die Konsequenz war ein Ausbau des Implantates und Spondylodese des ausgewachsenen Patienten. Somit lag hier die Komplikationsrate von konservativ assoziierten Komplikationen bezogen auf die Anzahl der untersuchten Personen $(n=17)$ bei $18 \%$ und bezogen auf die Anzahl der durchgeführten Verlängerungen $(343,12-19$ pro Patient) bei $0,9 \%$. 
Tabelle 3: Übersicht über die Anzahl und die Art der implantatspezifischen Komplikationen

\begin{tabular}{|l|l|}
\hline \multicolumn{2}{|c|}{ Implantatspezifische Komplikationen } \\
\hline Anzahl: & Art der Komplikation: \\
\hline 1 & Diskonnektion eines VEPTR ${ }^{2}-$ Rippenhakens \\
\hline 3 & Dislokation von VEPTR ${ }^{2}$-Rippenhaken \\
\hline 1 & Nicht ausfahrbares Implantat* \\
\hline 1 & Implantatversagen durch Diskonnektion nach übermäßiger Distraktion \\
\hline
\end{tabular}

*Nicht ausfahrbar bezeichnet ein totales Unvermögen, ein Implantat zu distrahieren. Ein Knacken des Implantates bei Verlängerung zeigt nur das Erreichen der maximalen Spannung und der in dem Moment maximal möglichen Distraktion an.

Nur bei einem Patienten hatten die Komplikationen negative Auswirkungen auf den Krümmungsverlauf. Bei allen weiteren Patienten war trotz ihrer Komplikationen kein Verlust der Krümmungskorrektur festzustellen. 


\subsection{Zusammenfassung der Ergebnisse}

Die vorliegenden Ergebnisse zeigten, dass mit dem bilateralen MAGEC@-Implantat mit einer Rippen zu Beckenkamm Fixierung und regelmäßigen ambulanten Distraktionen die primär erzielten Korrekturen der Skoliosekrümmung und des Beckenschiefstandes bei SMA-Kindern mit Wirbelsäulendeformität auch länger als vier Jahre gehalten werden konnten. Ebenso fand eine kontinuierliche Zunahme der Wirbelsäulenlänge statt. In diesem Patientenkollektiv wurde kein law of diminishing returns beobachtet, d.h. die Distraktion konnte auch jenseits von vier Jahren in dem untersuchten Kollektiv erfolgreich durchgeführt werden.

Ein Vergleich der Ergebnisse nach dorsaler Spondylodese bei SMA-Jugendlichen mit und

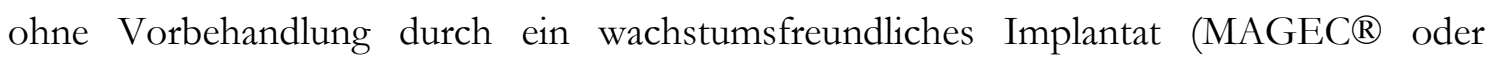
VEPTR $\left.{ }^{\circledR}\right)$ ergab eine bessere Skoliosekorrektur und einen geringeren Beckenschiefstand bei SMA-Kindern, die operativ vorbehandelt worden waren. Sie hatten eine signifikant bessere Reduktion der Skoliosekrümmung als die Vergleichsgruppe. Die Spinale Länge sowie Kyphose- und Lordosewinkel waren in beiden Gruppen nicht unterschiedlich. 


\section{Diskussion}

\subsection{Studienkollektiv}

Seit der Zulassung der MAGEC®-Therapie wurden Studien veröffentlicht, um aus den Beobachtungen in der klinischen Anwendung dieser Therapie die Effizienz und die Komplikationen der Implantate beziehungsweise des Verfahrens analysieren zu können (Hosseini et al. 2016; Keskinen et al. 2016; Lorenz et al. 2017; Ridderbusch et al. 2017; Hell et al. 2018; Lorenz et al. 2019). In einer aktuellen Studie (Akbarnia und Mundis 2019) wurden die magnetisch distrahierbaren Implantate ebenso effektiv wie die traditionellen Growing Rods in Bezug auf die Krümmungskorrektur und das Wirbelsäulenwachstum mit dem Vorteil der Reduktion der Operationen und damit der operationsbedingten Komplikationen beschrieben. Obwohl das MAGEC®-Implantat erstmals 2009 in Asien verwendet wurde (Cheung et al. 2012), seit 2011 in Europa genutzt wird (Hell et al. 2018) und seit 2014 in den USA zugelassen ist, liegen die Mehrzahl der Verlaufsbeobachtungszeiten der im Jahr 2019 veröffentlichen Studien bei nur etwas mehr als zwei Jahren (Lampe et al. 2019; Studer et al. 2019; Wu et al. 2019). Somit fehlen Studien zum Langzeitverlauf und zum Abschluss der Behandlung mit MAGEC®-Implantaten, was Hosseini und Akbarnia in einem Kommentar zur Studie von Cheung et al. (2019) feststellten (Hosseini und Akbarnia 2019).

Mit dem MAGEC®-Implantat werden Kinder mit einer progressiven Early Onset Scoliosis therapiert mit dem Ziel, die Skoliosekrümmung zu korrigieren und ein Thoraxinsuffizienzsyndrom (TIS) zu vermeiden (Campbell et al. 2004; Campbell und Smith 2007). Die zugrundeliegende Ätiologie kann dabei unterschiedlich sein. Wenn sehr heterogene Kollektive untersucht werden, bleibt bei den veröffentlichten Ergebnissen die Frage offen, ob der beobachtete Verlauf durch die Effektivität beziehungsweise das Versagen des Implantats erklärt werden kann oder ob die Ergebnisse von unterschiedlichen Diagnosen und Einflussfaktoren, wie zum Beispiel der Steifigkeit der Skoliose, beeinflusst waren. Beispielsweise bestand das Kollektiv der Studie von Studer et al. (2019) aus 30 EOSPatienten, von denen elf kongenital, zehn neuromuskulär, vier Syndrom-assoziiert und fünf idiopathisch bedingt waren.

Die vorliegende prospektive Studie untersuchte im Gegensatz zu den zahlreichen bisherigen Veröffentlichungen mit heterogenen EOS-Kollektiven (Lampe et al. 2019; Studer et al. 2019; Wu et al. 2019) nun ein homogenes Kollektiv von 17 Kindern im ausgeglichenen Geschlechterverhältnis mit SMA und neuromuskulärer Skoliose (Kohorte 1), welche in ihrer Wachstumsphase mit mitwachsenden Implantaten zur Krümmungskorrektur und zur TIS Prävention therapiert wurden. Als Fortführung und 
Erweiterung der Dissertation von Batoul Badwan (Badwan 2017; Lorenz et al. 2017), wurde das prospektive Patientenkollektiv von SMA-Kindern jetzt mit einer Verlaufsbeobachtungszeit von mehr als vier Jahren pro Patient (im Mittel 4,6 Jahre) im Langzeitverlauf beobachtet. Die Untersuchung von einem homogenen Kollektiv mit SMA und MAGEC®-System von mehr als vier Jahren Verlaufsbeobachtungszeit ist letztendlich von hohem wissenschaftlichen Interesse, derzeitig in dieser Arbeit einmalig und eignet sich hervorragend, um die Analyse der Effizienz der MAGEC®-Therapie im Langzeitverlauf mit möglichst wenigen Störfaktoren zu ermöglichen.

Neun Patienten des Kollektivs sind ausgewachsen und wurden mit einer Spondylodese versorgt, sodass die MAGEC®-Therapie anhand dieser Verläufe von Beginn bis zum Abschluss ausgewertet werden konnte. Der Abschluss der Behandlung mit mitwachsenden Implantaten ist ein aktuelles Diskussionsthema und es existieren noch keine einheitlich definierten, wissenschaftlich belegbaren Standards, weshalb das wissenschaftliche Interesse an Studien diesbezüglich hoch ist (Akbarnia und Mundis 2019; Hosseini und Akbarnia 2019). Es wurde lediglich von Kocyigit et al. (2017) in einer Vergleichsstudie zu verschiedenen Versorgungsmöglichkeiten nach Ende der Therapie mit mitwachsenden Implantaten belegt, dass es im Allgemeinen keine Option ist nur die Implantate zu entfernen. Derzeit werden die meisten Kinder und Jugendlichen mit skelettaler Reife bzw. am Ende der Wachstumsphase (ca. 14. Lebensjahr) durch eine dorsale Spondylodese definitiv versorgt (Flynn et al. 2013; Kocyigit et al. 2017; Akbarnia und Mundis 2019).

Die wissenschaftliche Betrachtung der Spondylodese erfolgte bisher entweder im Zusammenhang mit dem Abschluss der Behandlung nach mitwachsenden Implantaten (Flynn et al. 2013; Kocyigit et al. 2017) oder in Studien zur primären Versteifung ohne operativer Vorbehandlung (Zebala et al. 2011; Holt et al. 2017). Mit der Untersuchung gleichwertiger Kohorten von 14 SMA-Patienten mit Spondylodese und Vorbehandlung durch ein mitwachsendes Implantat (Kohorte 2b) im Vergleich zu 14 SMA-Patienten mit Spondylodese ohne operative Vorbehandlung (Kohorte 2a), wurden nun beide Therapiealternativen in gleichwertigen Gruppen direkt gegenübergestellt. Diese Art der Vergleichsanalyse ist derzeit für diese Art von Implantat und in diesem Patientenkollektiv bisher nicht erfolgt. 


\subsection{Veränderungen der radiologischen Parameter im Langzeitverlauf}

\subsubsection{Skoliosekrümmungswinkel im Langzeitverlauf}

Es wurde mehrfach gezeigt (Ridderbusch et al. 2017; Akbarnia und Mundis 2019), dass das MAGEC@-System zur Krümmungskorrektur ebenso effizient ist, wie traditionelle mitwachsende Systeme. Dies wurde zuletzt von Bekmez et al. (2019) durch eine Vergleichsstudie zweier identischer Gruppen kongenitaler EOS ( $n=10$ MCGR; n=10 TGR) bestätigt sowie in der Metaanalyse von Tang et al. (2019; n=186 MCGR; n=64 CGR) unter Beweis gestellt. Doch bisherige Studien zu traditionellen mitwachsenden Systemen, wie zum Beispiel von Sankar et al. (2011), sowie auch aktuelle Veröffentlichungen zu MAGEC@-Implantaten, beschrieben ein law of diminishing returns (Sankar et al. 2011; Lampe et al. 2019; Studer et al. 2019). Trotz der Fortführung der Therapie mit mitwachsenden Systemen wurde im Verlauf der Jahre ein abnehmendes Korrekturpotenzial beobachtet.

Zu Therapiebeginn konnte in vergleichbaren Studien (Lampe et al. 2019; Studer et al. 2019) genauso wie in dieser Studie zunächst eine signifikante Krümmungskorrektur durch die Primärimplantation des MAGEC®-Implantates festgestellt werden. Studer et al. (2019) korrigierten den durchschnittlichen Ausgangs-Cobb-Winkel ihres heterogenen Kollektivs $(\mathrm{n}=30)$ um $29 \%$ von $66^{\circ}$ auf $47^{\circ}$ und Lampe et al. (2019) berichteten von $46 \%$ initialer Korrektur des Skoliosekrümmungswinkels $(n=24)$. Allerdings startete das homogene Kollektiv der vorliegenden Studie $(n=17)$ mit durchschnittlich $70^{\circ}$ Cobb-Winkel und konnte auf $29^{\circ}$ um $59 \%$ signifikant $(\mathrm{p}<0,001)$ verbessert werden. Da SMA-Patienten weiche dehnbare neuromuskuläre EOS entwickeln, erklärt sich, dass in diesem homogenen Kollektiv mit flexiblen Krümmungen ein besonders gutes Korrekturpotenzial von 59 \% bei Primärimplantation beobachtet werden konnte.

Über den gesamten anschließenden Verlauf der Verlängerungen 1 bis 16 wurde der CobbWinkel des Studienkollektivs (Kohorte 1) konstant um $35^{\circ}\left( \pm 2^{\circ}\right)$ gegen den leichten Korrekturverlust des Körperwachstums gehalten. Im Gegensatz dazu beschrieben Studer et al. (2019) einen Korrekturverlust im Sinne eines law of diminishing returns mit jeder zusätzlichen Verlängerung und Lampe et al. (2019) errechneten einen Korrekturverlust von $5 \%$ nach durchschnittlich 42 Monaten Beobachtungszeit. Entgegen der Studien von Sankar et al. (2011), Studer et al. (2019) und Lampe et al. (2019) fand sich im untersuchten Kollektiv kein law of diminishing returns.

Dass die Flexibilität der Wirbelsäule trotz einer Behandlung mit mitwachsendem Implantat über Jahre erhalten bleibt, zeigten auch die Bendingaufnahmen in einer kleinen Anzahl von Patienten $(n=4)$ und das gute Korrekturpotenzial bei der Spondylodese in der vorbehandelten Kohorte (Kohorte 2b).

Die vorliegenden Ergebnisse über das Outcome der SMA-Spondylodese-Patienten mit Vorbehandlung durch mitwachsende Implantate (Kohorte 2b) ergaben eine signifikant $(\mathrm{p}=0,037)$ bessere Skoliosekrümmungskorrektur nach Spondylodese als bei SMA-Patienten 
ohne Vorbehandlung (Kohorte 2a). Der Krümmungswinkel verringerte sich bei den Patienten mit Vorbehandlung um $54 \%$ von $71^{\circ}$ vor Spondylodese auf $33^{\circ}$ danach. Im Vergleich dazu betrug die Korrektur bei den SMA-Patienten ohne Vorbehandlung $50 \%$ (von $104^{\circ}$ auf $50^{\circ}$ Cobb-Winkel). Das Korrekturpotenzial dieser weichen Skoliosen war jedoch in beiden Gruppen besser als das von Flynn et al. (2013) in ihrer Studie zum Abschluss der Behandlung mit mitwachsenden Implantaten. Die Auswertung von Flynn et al. (2013) von 79 Versteifungen aus einem heterogenen EOS-Kollektiv nach Therapie mit mitwachsendem Implantat ergab ein Korrekturpotenzial von $<50 \%$ bei Spondylodese.

Weiterhin untersuchten Flynn et al. (2013) die Wirbelsäulenflexibilität nach Langzeittherapie und beobachteten dabei in ihrer Studie, dass von 79 EOS-Patienten, die durchschnittlich fünf Jahre mit mitwachsenden Implantaten therapiert waren nur noch elf bei Spondylodese eine mobile Wirbelsäule hatten. Bei 68 Patienten waren die Wirbelsäulen nur noch wenig flexibel oder steif, woraus das Korrektionspotenzial von $<50 \%$ bei Spondylodese resultierte. Das heterogene Kollektiv lässt nicht erkennen, ob die steifen Skoliosen nun Therapie bedingt oder Ätiologie bedingt waren (Flynn et al. 2013).

Bevor die SMA-Patienten der vorliegenden Studie zum Wachstumsabschluss eine operative Versteifung erhielten, wurde das mitwachsende Implantat zunächst explantiert und die Spondylodese in einigen Wochen Abstand geplant. Dieses zweizeitige Verfahren wurde zur Prävention von Bakterienbesiedlung und Infektionen gewählt, da $40 \%$ der Patienten beziehungsweise $46 \%$ der Patienten aus den Studien von Plaass et al. (2016) und Wagner et al. (2018) besiedelte Implantate hatten. Es ermöglichte die Untersuchung der Flexibilität der Wirbelsäulen der Patienten zwischen dem Ausbau und der Spondylodese, welche anhand von präoperativ durchgeführten Bendingaufnahmen erfolgte. Zwischen den beiden Interventionen veränderte sich der Skoliosekrümmungswinkel der Patienten mit wachstumsfreundlichem Implantat von $44^{\circ}$ bei Ausbau auf $71^{\circ}$ vor Spondylodese. Die Analyse der präoperativen Bendingaufnahmen eines Teilkollektivs von vier Patienten mit Vorbehandlung (Kollektiv 2b) zeigte ein Korrekturpotenzial von $25 \%$ (von $71^{\circ}$ auf $53^{\circ}$ Cobb-Winkel). Im Vergleich dazu zeigte sich bei den SMA-Patienten ohne Vorbehandlung (Kohorte 2a) in den präoperativen Bendingaufnahmen ( $n=14)$ eine Aufdehnung von $104^{\circ}$ auf $66^{\circ}$, was einer Korrektur von $37 \%$ entsprach. Somit konnte nach Vorbehandlung eine Teilflexibilität der Wirbelsäule nachgewiesen werden, die jedoch unter der des unbehandelten Kollektivs lag. Durch die anschließende Spondylodese erfolgten die bereits genannten Korrekturen von $71^{\circ}$ auf $33^{\circ}$ um $54 \%$ im vorbehandelten Kollektiv und von $104^{\circ}$ auf $50^{\circ}$ um $50 \%$ im unbehandelten Kollektiv.

Bisherige Studien zur primären Versteifung von unbehandelten SMA-Kollektiven kamen auf bessere Krümmungskorrekturen (Zebala et al. 2011; Holt et al. 2017) als die Ergebnisse der vorliegenden Arbeit. Zebala et al. (2011) analysierten retrospektiv 22 SMA-Patienten, die bei einer primären Versteifung mit Luque-Galveston Instrumentierung von im Mittel $76,5^{\circ}$ auf $29,8^{\circ}$ um $61,1 \%$ korrigiert werden konnten und bei Holt et al. (2017) waren es 16 
SMA-Patienten, bei denen sich der Cobb-Winkel bei primerer Versteifung mit Luque Stangen Konstruktion von $78^{\circ}$ auf $25^{\circ}$ um $68 \%$ verbesserte. Der Unterschied zu der vorliegenden Studie war das Alter zum Versteifungszeitpunkt, welches bei Zebala et al. (2011) bei durchschnittlich 8,4 Jahren und bei Holt et al. (2017) bei durchschnittlich 9,8 Jahren lag. Im Vergleich dazu lag das Durchschnittsalter bei Spondylodese des Kollektivs ohne Vorbehandlung der vorliegenden Studie (Kollektiv 2a) bei 13,7 Jahren. Obwohl die Krümmung der Wirbelsäule bei SMA zu den weichen flexiblen Skoliosen zählt, lässt die Gegenüberstellung der Krümmungskorrekturergebnisse der primären Versteifungen bei SMA im Alter von acht bis zehn Jahren zu den Ergebnissen mit 13 bis 14 Jahren eine Verminderung der Wirbelsäulenflexibilität im natürlichen Verlauf ohne operative Eingriffe vermuten. Ebenso liegt vermutlich eine stärkere Wirbelkörperdeformierung in der älteren Gruppe vor.

Zum Verlust der Wirbelsäulenflexibilität bei der Therapie mit wachstumsfreundlichen Implantaten beschrieben Sankar et al. (2011) ein law of diminishing returns, welches sie mit Autofusionsprozessen, Ossifikationen und Vernarbungen durch multiple Operationen erklärten (Sankar et al. 2011; Groenefeld und Hell 2013). Von der Behandlung des Studienkollektivs mit MAGEC®-Implantaten versprach man sich einen Teilerhalt der Flexibilität der Wirbelsäule durch Vermeidung von Operationen und damit Vernarbungen, wirbelsäulenferner Befestigung und physiologischeren Verlängerungen im Abstand von drei Monaten (Hell et al. 2018). In aktuellen Studien gibt es zu dem law of diminishing returns bei

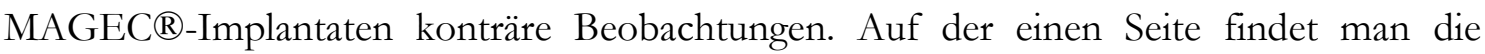
Studienergebnisse zu einem law of diminishing returns bereits nach zwei Jahren MAGEC®Therapie (Lampe et al. 2019; Studer et al. 2019), auf der anderen Seite gibt es Studien, die keinen Korrekturverlust mit Fortführung der Therapie feststellten (Gardner et al. 2017; Lorenz et al. 2019), was die Frage nach den Ursachen des Korrekturverlustes aufwirft.

Bezüglich der Kausalitätsfrage, ob der Flexibilitätserhalt nun ein Effekt des MAGEC®Systems sein kann, lässt sich vermuten, dass dies zum Teil der Fall ist, aber auch das besondere Kollektiv der SMA-Patienten im Langzeitverlauf relativ weiche Skoliosen behält. Weitere prospektive Analysen mit anderen homogenen Krankheitsbildern müssen noch zeigen, wie sich der Langzeitverlauf anderer EOS-Entitäten mit MAGEC®-Implantaten verhält und ob trotz MAGEC®-Therapie mit wirbelsäulenferner Befestigung Wirbelsäulenverknöcherungen entstehen oder nicht.

Besondere Aufmerksamkeit sollte auch den Langzeitstudien größerer Kollektive gelten, um zu sehen, ob auch in großen Stichproben gute Korrekturen gehalten werden können oder sich weitere Ursachen für ein law of diminishing returns auftun. Beispielsweise analysierten Wu et al. (2019) 116 EOS-Patienten metaanalytisch. Sie veröffentlichten vorläufige Ergebnisse von einer Primärkorrektur des Krümmungswinkels von im Mittel 60,1 ${ }^{\circ}$ auf $35,4^{\circ}$, die nach 
einer Beobachtungszeit von 23-61 Monaten bei durchschnittlich 36,9 $9^{\circ}$ fast gehalten werden konnte (Wu et al. 2019).

\subsubsection{Beckenschiefstand im Langzeitverlauf}

Der Beckengradstand ist für Kinder mit neuromuskulärer Skoliose für eine gute Sitzposition unerlässlich.

Im Jahr 2010 zeigten Hasler et al. (2010) an ihrem Kollektiv von Kindern mit VEPTR®Stangen und Implantatbefestigung mit Rippen- zu Beckenkamm Fixierung, dass der Beckenschiefstand gut korrigiert werden konnte, bemerkten jedoch, dass sie Beckenschiefstände unter $30^{\circ}$ um $80 \%$ verbessern konnten, Beckenschiefstände über $30^{\circ}$ dagegen nur um $20 \%$. Die Schlussfolgerung war die Vermutung, schwere Beckenschiefstände mit Hakenbefestigung nicht ausreichend korrigieren zu können, und dass eine rigidere Alternative für diese Fälle notwendig sein könnte (Hasler et al. 2010). Die Studie von Cottrill et al. (2019; n=24 EOS, 2 Jahre Follow-up) und die Studie von Brooks et al. (2016; n=38 EOS, 4 Jahre Follow-up) bewerteten die Verschraubung des Beckens als beste Option für die distale Implantatbefestigung, um gute Korrekturen zu erreichen und zu halten (Brooks et al. 2016; Cottrill et al. 2019).

Da die meisten MAGEC®-Studien eine Pedikelschraubenbefestigung der MAGEC®Implantate nutzten, wurden Daten zum korrigierenden Einfluss auf das Becken nicht angegeben (Lampe et al. 2019; Studer et al. 2019).

Die vorliegende Arbeit zeigte, dass die Eifeltower Construction mit Rippen- und Beckenhakenbefestigung der MAGEC®-Stangen eine gute Korrektur der Beckenkippung erreichen konnte. Primär konnte eine durchschnittliche Beckenkippung von $17,7^{\circ}$ auf $4,5^{\circ}$ $(p=0,16)$ korrigiert werden und im gesamten Beobachtungsverlauf um 4,6 gehalten werden. Zum Abschluss der Behandlung fand sich in der Kohorte mit Vorbehandlung (Kohorte 2b) ein geringerer Beckenschiefstand von 5,7 gegenüber der Kohorte ohne Vorbehandlung (Kohorte 2a) mit 12,4 $4^{\circ}$ Durch die Eifeltower Construction wurde über die Wachstumsphase ein korrigierender Einfluss auf das Becken genommen, welcher in der Kohorte ohne Vorbehandlung (Kohorte 2a) fehlte.

In dem untersuchten Kollektiv zur Analyse des MAGEC®-Langzeitverlaufes konnten nebenbefundlich bei zwölf von 17 Kindern (Kollektiv 1) ein- oder beidseitige Hüftgelenksluxationen festgestellt werden, was ebenfalls als ein Faktor für einen Beckenschiefstand zu werten ist. Dass eine SMA häufig mit einer Hüftluxation einhergeht, beschrieben schon Granata et al. im Jahr 1990 (Granata et al. 1990). In der Studie von Pate und Shapiro aus dem Jahr 2015 zur Dokumentation neuromuskulärer Deformitäten von 211 Patienten ( $n=110$ Duchenne Muskeldystrophie, $n=49$ SMA, $n=26$ Zerebralparese) konnte eine zusammenhängende Trias von Wirbelsäulen-, Becken- und Hüftdeformation beobachtet werden (Patel und Shapiro 2015). Da die Patienten mit neuromuskulärer Erkrankung jedoch trotz der Hüftluxation als beschwerdefrei galten, wurde auch in weiteren Arbeiten 
keine operative Behandlung empfohlen (Sporer und Smith 2003; Zenios et al. 2005; Canavese und Sussman 2009). Hingegen wurden durch den Beckenschiefstand bedingte Symptome wie Schmerzen und Probleme mit der Sitzposition beschrieben. Canavese und Sussmann (2009) behandelten diese Beschwerden in ihrer Studie zum Hüftmanagement bei neuromuskulären Erkrankungen genauso wie in der vorliegenden Studie durch die Korrektur und Stabilisierung der Wirbelsäulendeformität, welche einen korrigierenden Einfluss auf das Becken hatte (Canavese und Sussman 2009).

\subsubsection{Spinale Länge, Kyphose- und Lordosewinkel im Langzeitverlauf}

Tang et al. (2019) konnten zeigen, dass das MAGEC®-System nicht nur die Skoliosekrümmung effektiv korrigierte, sondern ebenfalls das Wirbelsäulenwachstum effektiv förderte. In der vorliegenden Studie wurde ein signifikanter $(p<0,001)$ Zuwachs der Wirbelsäulenlänge um $53 \mathrm{~mm}$ von $236 \mathrm{~mm}$ auf $289 \mathrm{~mm}$ durch die operative Aufrichtung der Wirbelsäulendeformität erreicht, welcher sich im Verlauf mit jeder Verlängerung kontinuierlich um 4,3 mm weiter vergrößerte (Lorenz et al. 2019). Vergleichbare Untersuchungen wie die Metaanalyse von Wu et al. (2019) von 116 EOSPatienten beschrieben einen primären Zuwachs um $27 \mathrm{~mm}$ oder die Studie von Lampe et al. (2019) beobachteten an 24 EOS-Patienten einen primären Zuwachs von im Mittel $25 \mathrm{~mm}$, allerdings nur eine weitere Längenzunahme von 1,6 mm pro Verlängerung. Die gute Primärkorrektur des untersuchten homogenen SMA-Kollektivs lässt sich im Vergleich zu dem schlechteren primären Längenzuwachs der heterogenen Kollektive aus vergleichbaren Studien durch die weichen SMA-Skoliosen erklären. Der weitere Verlauf der erwarteten und reellen Wirbelsäulenlänge wurde von Lorenz et al. (2019) untersucht, der eine 94,4 prozentige reelle, radiologisch gemessene Längenzunahme gegenüber der

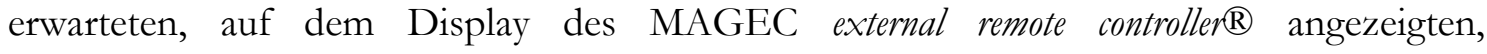
Längenzunahme zeigen konnte. Die Effektivität des MAGEC®-Implantates zum Erreichen einer kontinuierlichen Längenzunahme bei SMA-Kindern wurde durch diese Daten bestätigt.

Mitwachsende Wirbelsäulenimplantate haben durch die vorhandene Zwei-Punkt-Fixierung häufig das Problem, dass Rotationsdeformitäten und das sagittale Profil schwer zu beeinflussen sind. In der Literatur zum Verlauf des sagittalen Profils bei mitwachsenden Implantaten beobachteten Chen et al. (2017a) in einer Vergleichsstudie von elf VEPTR®Patienten mit Rippen- und Beckenhakenfixierung zu 22 Growings-rods-Patienten mit Pedikelschraubenfixierung eine bessere Kyphosekorrektur und weniger Korrekturverlust nach zwei Jahren Verlaufsbeobachtungszeit bei den Growing-rods-Patienten mit $44 \%$ Primärkorrektur der Kyphose von $56^{\circ}$ auf $32^{\circ}$ und $11 \%$ Korrekturverlust auf $38^{\circ}$ Kyphosewinkel gegenüber den Patienten mit VEPTR®-Systemen mit $23 \%$ Primärkorrektur von $52^{\circ}$ auf $40^{\circ}$ und $46 \%$ Korrekturverlust auf $64^{\circ}$ Kyphosewinkel nach zwei Jahren. Allerdings zeigte sich bei den VEPTR®-Patienten weniger Proximale Junktionale Kyphose, sodass Chen et al. (2017a) zu dem Schluss kamen, zur Korrektur des 
sagittalen Profils mit hyperkyphotischer Deformität bei EOS die Growing-rods-Behandlung mit Pedikelschraubenbefestigung zu empfehlen, obwohl dem VEPTR ${ }-S y s t e m$ mit Rippen- und Beckenhakenfixierung der weichere Übergang zur nicht instrumentierten Wirbelsäule zugutegehalten werden musste. Mit einer weiteren Studie zeigten Chen et al. (2017b) die Effektivität der Growing rods eine Hyperkyphose zu korrigieren, aber auch eine zu Beginn normale Kyphose über den Behandlungsverlauf zu halten. Die vergleichbare MAGEC®-Literatur zum Beispiel die Metaanalyse von Wu et al. (2019) anhand von 116 EOS-Patienten mit mindestens zwei Jahren Follow-up beobachteten eine Zunahme des Kyphosewinkels im Verlauf. Ihre Ergebnisse zeigten eine Primärkorrektur von $38^{\circ}$ auf 26,1 $1^{\circ}$ durch MAGEC®-Stangen mit Pedikelschraubenbefestigung, welche sich nach zwei Jahren auf $36^{\circ}$ veränderte (Wu et al. 2019).

Während in der vorliegenden Arbeit mit der Implantation primär eine signifikante Korrektur des sagittalen Profils mit einer Reduktion des Kyphosewinkels von 44,5 auf $34,3^{\circ} \quad(\mathrm{p}<0,01)$ erreicht werden konnte, undulierte der Wert um $37^{\circ}$ bei den Verlängerungen. Entgegen der Beobachtungen von Chen et al. (2017) und Wu et al. (2019) zeigten diese Ergebnisse eine vergleichbare Effektivität von Growing rods mit Pedikelschraubenbefestigung und MAGEC®-Systemen mit Eifeltower Construction, da trotz Rippen- und Beckenhakenbefestigung kein progredienter Korrekturverlust zu beobachten war. Auf den Lordosewinkel konnte kein signifikanter Einfluss genommen werden. Er veränderte sich von $28,4^{\circ}$ auf $30,8^{\circ}$ bei Implantation und undulierte im Verlauf um $25^{\circ}$.

Die Auswertung des sagittalen Profils des Studienkollektivs nach Durchführung der Spondylodese zeigte, dass sich die Spinale Länge, der Kyphose- und Lordosewinkel gleichwertig in beiden Vergleichsgruppen mit oder ohne Vorbehandlung veränderten. Dies lässt sich zum Beispiel auch in der Arbeit zum Abschluss der Behandlung von EOS nach mitwachsenden Systemen zur Skoliosekorrektur von Pizones et al. (2018) finden, die eine gute Kontrolle des sagittalen Profils bei EOS-Patienten nach der dorsalen Spondylodese mit Verbesserung von Spinaler Länge und Kyphose zeigte. Einschränkend muss auf die Altersabhängigkeit des Kyphos- und Lordosewinkels hingewiesen werden (Giglio und Volpon 2007), welcher bei allen Korrekturbeschreibungen nicht berücksichtigt wurde. Somit kann eine Zunahme der Kyphose beispielweise auch eine Annäherung an den altersentsprechenden Normwert bedeuten. 


\subsection{Komplikationen}

Insgesamt traten in dem untersuchten Kollektiv im Untersuchungszeitraum keine lebensbedrohlichen Komplikationen auf, jedoch war vor allem die Komplikationsrate der operationsassoziierten Komplikationen mit 47 \% bezogen auf die Anzahl der Patienten ( $\mathrm{n}=17$ ) nicht unerheblich. Die Komplikationsrate von konservativ assoziierten Komplikationen war deutlich niedriger. Bezogen auf die Anzahl der untersuchten Personen ( $n=17)$ lag sie bei $18 \%$ und bezogen auf die Anzahl der durchgeführten Verlängerungen (343, 12-19 pro Patient) bei $0,9 \%$. Unter den Komplikationen fanden sich am häufigsten mechanische beziehungsweise implantatassoziierte Komplikationen, wie zum Beispiel diskonnektierte Rippenhaken. In der weiteren Literatur (Beaven et al. 2018; Thakar et al. 2018) sind ebenfalls relativ hohe Komplikationsraten mechanischer Probleme beschrieben. Eine Auswertung von 336 Patienten mit durchschnittlicher Beobachtungszeit von 29,7 Monaten durch Thakar et al. (2018) ergab eine durchschnittliche Komplikationsrate von 44,5 \%, wobei sich am häufigsten Befestigungsanker lösten, die Stäbe brachen oder die Implantate versagten. Auch Beaven et al. (2018) fanden mit $29 \%$ eine hohe Rate von mechanischem Versagen in einer retrospektiven Studie von 28 Kindern mit MAGEC®-Behandlung über zwei Jahre. Daraus ergibt sich die Frage, ob sich die Ursache der implantatassoziierten Komplikationen bei den Implantaten oder in der Anwendung finden lässt. Dazu wurde anhand der Ergebnisse der Studie von Lorenz et al. (2019) ein hoher BMI als Risikofaktor für MAGEC®-Therapieversagen festgestellt. Laut Lorenz et al. (2019) waren von den beobachteten Distraktionsversagen in 13 Fällen zehn Patienten übergewichtig. Zu viel Gewicht und Spannung auf dem Implantat in Kombination mit einer längeren Strecke vom externen Controller zum Stangengewinde durch mehr Weichgewebe erschwerte die Verlängerungen (Lorenz et al. 2019). Diese Beobachtung gleicht denen der vorliegenden Studie, da die Komplikationen nur bei einem Patienten des Kollektivs negative Auswirkungen auf den Krümmungsverlauf hatten und es sich in diesem Fall um einen adipösen Patienten handelte. Bei allen weiteren Patienten der vorliegenden Studie war trotz ihrer Komplikationen kein Verlust der Krümmungskorrektur festzustellen.

Eine Erklärung für die im Allgemeinen niedrigere Komplikationsrate des Studienkollektivs bei Verlängerungen findet sich vielleicht bei den Patientenkollektiven. Im homogenen SMA-Kollektiv dieser Arbeit fanden sich wenige mechanische Komplikationen bei Distraktionen, sodass weiche Skoliosen dieser normalgewichtigen und untergewichtigen Kinder komplikationsärmer mit MAGEC®-Stangen therapiert werden konnten. Das häufigere Auftreten von mechanischen Komplikationen in heterogenen Kollektiven, wirft die Frage auf, ob das MAGEC®-System für alle EOS-Ätiologien geeignet ist. Eine genaue Analyse der einzelnen Komplikationsraten verschiedener Entitäten und Krankheitsbildern wäre diesbezüglich von besonderem Interesse, um Patienten individuell beraten zu können. 


\subsection{Limitationen der Studie und Ausblick}

In der vorliegenden Arbeit konnte gezeigt werden, dass die MAGEC®-Implantate mit Rippen- und Beckenhakenfixierung bei Kindern mit SMA effektiv zur Therapie von Wirbelsäulendeformitäten ohne signifikanten Korrekturverlust im Sinne eines law of diminishing returns im Langzeitverlauf eingesetzt werden konnten. Neben den Vorteilen eines homogenen Kollektivs durch Vermeidung von Störfaktoren, bleiben Nachteile wie die reduzierte Übertragbarkeit und Aussagekraft der Ergebnisse auf die Allgemeinheit der EOS-Patienten. Die guten Ergebnisse dieses Kollektivs lassen sich vermutlich auf ein konsequentes Nachbehandlungsschema und die weiche flexible Skoliosekrümmung der SMA-Kinder zurückführen. Folglich steht die differenzierte Untersuchung, Analyse und Betrachtung von Wirbelsäulendeformitäten anderer Genese im Langzeitverlauf noch aus.

Weiterhin sind ein Minimum von vier Jahre Verlaufsbeobachtung eher ein „kurzer“ Langzeitverlauf. Jedoch wird dieses Follow-up durch die Indikation für die Anwendung von mitwachsenden Implantaten bedingt. Das heißt, da das MAGEC®-Implantat als mitwachsendes Implantat zur Krümmungskorrektur während der Wachstumsphase gedacht ist und die Patienten nach Wachstumsabschluss mit einer Spondylodese definitiv versorgt werden, könnten längere Beobachtungszeiten als die vorliegende nur durch eine Implantation des MAGEC®-Systems im jüngeren Alter bei medizinisch begründeter Indikation erreicht werden (hier im Mittel 7,4 Jahre bei Primärimplantation). Im Hinblick auf den Verlauf nach Spondylodese kann eine weitere längere Beobachtung des Kollektivs weiterhin interessant sein. Beispielsweise mit der Fragestellung, ob die gut korrigierte Beckenkippung im Verlauf nach Spondylodese ohne Beckenverschraubung gehalten werden kann, oder ob die Pedikelschrauben nach Spondylodese in dem osteoporotischen Knochen der SMAPatienten langfristig halten, auswandern oder sich eine sekundärer Korrekturverlust einstellt.

Schließlich waren die Röntgenaufnahmen im Stehen, beispielsweise als Standard zur Messung des Beckenschiefstandes, bei SMA-Patienten mit fehlender Stehfähigkeit nicht möglich, sodass die Patienten im Sitzen geröntgt wurden. Trotz speziell angefertigtem Röntgenstuhl wirken sich kleine Unterschiede in der Sitzposition direkt vor allem auf die Beckenkippung aus, was leichte Schwankungen im Datenverlauf erklärt und als Fehlerquelle bedacht werden muss.

Um genauer herauszufinden, ob die Vermeidung des Korrekturverlustes (law of diminishing returns) allein an der Skoliosentität SMA mit weicher Skoliose liegt, fehlen neben der Langzeitanalyse anderer Skolioseentitäten außerdem Vergleichsstudien zu den unterschiedlichen Verankerungsarten und dem Einfluss der Knochenqualität. Es wäre somit von wissenschaftlichem Interesse, nach Langzeittherapie mit MAGEC®-Implantaten zu erörtern, ob ohne Verschraubung an der Wirbelsäule tatsächlich keine Ossifikationen und Autofusionen zu finden sind und mit welcher Verankerungsart die beste Langzeitkorrektur bei möglichst gutem Erhalt der Flexibilität der Wirbelsäule erreicht werden kann. 
Darüberhinaus würde eine Studie über den Einfluss der Knochenqualität helfen, um Patienten entsprechend ihrer Osteoporosetherapie besser beraten zu können.

Durch die zunehmenden kausalen Behandlungskonzepte von Kindern mit SMA (Shorrock et al. 2018; Waldrop und Kolb 2019) mit der Gentherapie Zolgensma (onasemnogene abeparvovec) (Al-Zaidy et al. 2019) und der intrathekalen Oligonukleotide Spinraza ${ }^{\circledR} /$ Nusinersen Injektion (Biogen 2017; Mercuri et al. 2018; Pechmann et al. 2019) wird sich die Wirbelsäulendeformität bei SMA-Kindern voraussichtlich ändern. Die derzeitigen Beobachtungen des Studienkollektivs im klinischen Alltag lassen Vermutungen anstellen, dass beispielsweise SMA-Patienten in Zukunft länger leben könnten, und dass der Krankheitsverlauf schwerbetroffener Patienten mit SMA-Typ 0 und I, welche in der Vergangenheit frühzeitig verstarben, milder verlaufen könnte. Das hätte wahrscheinlich zur Folge, dass bei diesen schwer betroffenen Kindern eine frühere und stärkere Wirbelsäulendeformität auftreten könnte. Für die aktuelle intrathekale Therapie ist eine Vermeidung von starken Wirbelsäulendeformitäten wichtig, um eine problemlose Lumbalpunktion zu ermöglichen (Lorenz et al. 2018). Somit werden derzeit die Studien zum Krankheitsverlauf von SMA-Kindern unter medikamentöser Behandlung mit großem Interesse an dem zukünftigen klinischen Bild eines SMA-Patienten unter medikamentöser Therapie mit Spannung weiter verfolgt (Mercuri et al. 2018; Shorrock et al. 2018; Waldrop und Kolb 2019). 


\section{$5 \quad$ Zusammenfassung}

In der vorliegenden Arbeit wurde in einer prospektiven Kohortenstudie der Langzeitverlauf von operativ versorgten Kindern mit Spinaler Muskelatrophie (SMA) und Wirbelsäulendeformität analysiert. Zur Korrektur der Wirbelsäulendeformität wurde das Kollektiv mit paraspinal verankerten Magnetic-Expension-Control (MAGEC®)-Implantaten versorgt und länger als vier Jahre beobachtet. Bei einem Teilkollektiv wurden die Ergebnisse der definitiven dorsalen Wirbelsäulenfusion mit denen von vorher nicht behandelten SMA-Kindern verglichen.

Das MAGEC®-System ist ein von extern durch Magnet zu verstellendes Implantat zur Behandlung kindlicher Wirbelsäulendeformitäten im Wachstumsalter. Durch die externe Steuerung und Vermeidung von Operationen, kann ein kurzzeitiger Verlängerungsrhythmus gewählt werden. Dies wirft die Frage auf, ob hierdurch das sogenannte law of diminishing returns, eine Verschlechterung der Distraktion im Langzeitverlauf, vermieden werden kann.

Das Ziel der vorliegenden Studie war eine Aussage über die Effizienz der MAGEC®Therapie im Langzeitverlauf zu treffen und diese Ergebnisse auf ein law of diminishing returns zu überprüfen.

17 SMA-Patienten wurden prospektiv mehr als vier Jahre (im Mittel 4,6 Jahre) im Verlauf beobachtet und ihre Röntgenbilder sowie ihre medizinischen Daten ausgewertet. Neun Patienten bekamen zum Wachstumsabschluss eine Spondylodese, sodass sie von Beginn bis Abschluss der Therapie verfolgt werden konnten. Insgesamt wurden 343 Distraktionen erfasst, davon 12-19 pro Patient. Die daraus gewonnenen Langzeitergebnisse wurden im Hinblick auf die Auswirkungen der Therapie auf die knöcherne Deformität, die Komplikationen und das Auftreten eines law of diminishing returns analysiert.

Ein weiteres Ziel der vorliegenden Arbeit war die Analyse, ob SMA-Kinder mit Vorbehandlung bessere Ergebnisse zum Endpunkt der Behandlung, also nach einer dorsalen Spondylodese, zeigten.

14 SMA-Patienten ohne operative Vorbehandlung wurden 14 Patienten mit Vorbehandlung durch ein mitwachsendes Implantat gegenübergestellt, welche im mittleren Alter von 13,7 Jahren beziehungsweise 13,8 Jahren dorsal mit einer Spondylodese versteift wurden. Anhand radiologischer Auswertungen wurde die Deformitätenkorrektur analysiert.

Die Ergebnisse zeigten eine signifikante Primärkorrektur des Krümmungswinkels nach Cobb von durchschnittlich $70^{\circ}$ auf durchschnittlich $29^{\circ}$ bei Implantation der wachstumsfreundlichen Implantate. Von der ersten Verlängerung bis nach vier Jahren Therapieverlauf konnte ein Krümmungswinkel von $35^{\circ}\left( \pm 2^{\circ}\right)$ konstant gehalten werden. Dabei wurde kein law of diminishing returns festgestellt. Komplikationen hatten nur in einem Fall eine negative 
Auswirkung auf den Krümmungsverlauf, wobei ein Implantat bei einem adipösen Patienten aufgrund von zu viel Spannung nicht extern distrahierbar war und dieser zweimal operativ verlängert werden musste. Die Rate der operationsassoziierten Komplikationen lag bei $47 \%$ (zum Beispiel Infekt), die der konservativen Komplikationen jedoch vergleichsweise geringer bei $18 \%$.

Die Gegenüberstellung der Spondylodeseergebnisse von nicht- zu vorbehandelten SMAKindern zeigte, dass sich der Krümmungswinkel bei den Patienten mit Vorbehandlung signifikant mehr um $54 \%$ von $71^{\circ}$ auf $33^{\circ}$ Krümmungswinkel im Vergleich zu $50 \%$ von $104^{\circ}$ auf $50^{\circ}$ bei SMA-Patienten ohne Vorbehandlung verringerte. Auch der Beckenschiefstand zeigte in der Kohorte mit Vorbehandlung einen geringeren Wert von 5,68 gegenüber der Kohorte ohne Vorbehandlung mit 12,4 . Die Spinale Länge, der Kyphosewinkel und der Lordosewinkel veränderten sich in gleicher Weise in beiden Kohorten. Die Analyse von Bendingaufnahmen vor Spondylodese bestätigte eine erhaltende Teilflexibilität trotz operativer Vorbehandlung.

Die Studienergebnisse zeigten, dass die MAGEC®-Therapie eine probate Therapiemethode für SMA-Kinder mit neuromuskulärer Skoliose zur Krümmungskorrektur und Kontrolle des Beckengradstandes auch im Langzeitverlauf von mehr als vier Jahren ist und bis zum Abschluss der Behandlung gehalten werden konnte. Ein law of diminishing returns konnte für das hier analysierte Kollektiv nicht beobachtet werden.

Dass SMA-Kinder tatsächlich von der Therapie durch ein mitwachsendes Implantat profitierten, zeigte sich im signifikant besseren Ergebnis der Skoliosekorrektur nach dorsaler Spondylodese im Vergleich zu SMA-Kindern ohne operative Vorbehandlung. 


\section{$6 \quad$ Literaturverzeichnis}

Ahmad A, Subramanian T, Panteliadis P, Wilson-Macdonald J, Rothenfluh DA, Nnadi C (2017): Quantifying the „law of diminishing returns“ in magnetically controlled growing rods. Bone Jt J 99-B, 1658-1664

Akbarnia BA, Mundis GM (2019): Magnetically controlled growing rods in early onset scoliosis. Orthop $\underline{48}, 477-485$

Al-Zaidy SA, Kolb SJ, Lowes L, Alfano LN, Shell R, Church KR, Nagendran S, Sproule DM, Feltner DE, Wells C et al. (2019): AVXS-101 (Onasemnogene Abeparvovec) for SMA1: Comparative Study with a Prospective Natural History Cohort. J Neuromuscul Dis $\underline{6}, 307-317$

Arakawa R, Arakawa M, Kaneko K, Otsuki N, Aoki R, Saito K (2016): Imaging Flow Cytometry Analysis to Identify Differences of Survival Motor Neuron Protein Expression in Patients With Spinal Muscular Atrophy. Pediatr Neurol 61, 70-75

Aslan C, Olgun ZD, Ayik G, Karaokur R, Ozusta S, Demirkiran GH, Unal F, Yazici M (2019): Does Decreased Surgical Stress Really Improve the Psychosocial Health of Early-onset Scoliosis Patients?: A Comparison of Traditional Growing Rods and Magnetically-controlled Growing Rods Patients Reveals Disappointing Results. Spine 44, E656-E663

Badwan B: Die Behandlung der kindlichen Skoliose bei spinaler Muskelatrophie mit extern zu kontrollierenden magnetischen Implantaten. Med. Diss. Göttingen 2017

Beaven A, Gardner AC, Marks DS, Mehta JS, Newton-Ede M, Spilsbury JB (2018): Magnetically Controlled Growing Rods: The Experience of Mechanical Failure from a Single Center Consecutive Series of 28 Children with a Minimum Follow-up of 2 Years. Asian Spine J 12, 794-802

Bekmez S, Afandiyev A, Dede O, Karaismailoğlu E, Demirkiran HG, Yazici M (2019): Is Magnetically Controlled Growing Rod the Game Changer in Early-onset Scoliosis? A Preliminary Report. J Pediatr Orthop 오, e195-e200

Biogen (2017): Presseinformation Spinraza ${ }^{\circledR}$ (Nusinersen) erhält als erste Therapie der spinalen Muskelatrophie (SMA) die Zulassung in Europa 01.06.2017. pdf Datei https://www.initiative-sma.de/downloads/, abgerufen am 02.05.2019

Bowen RE, Scaduto AA, Banuelos S (2008): Decreased body mass index and restrictive lung disease in congenital thoracic scoliosis. J Pediatr Orthop 28, $665-668$

Bozorg Qomi S, Asghari A, Salmaninejad A, Mojarrad M (2019): Spinal Muscular Atrophy and Common Therapeutic Advances. Fetal Pediatr Pathol $\underline{38}$, 226-238

Brooks JT, Jain A, Sanchez-Perez-Grueso F, Skaggs DL, Thompson GH, Akbarnia BA, Sponseller PD, Growing Spine Study Group (2016): Outcomes of Pelvic Fixation in Growing Rod Constructs: An Analysis of Patients With a Minimum of 4 Years of Follow-up. Spine Deform $\underline{4}, 211-216$ 
Brzustowicz LM, Lehner T, Castilla LH, Penchaszadeh GK, Wilhelmsen KC, Daniels R, Davies KE, Leppert M, Ziter F, Wood D (1990): Genetic mapping of chronic childhood-onset spinal muscular atrophy to chromosome 5q11.2-13.3. Nature $\underline{344}$, 540-541

Burnett BG, Crawford TO, Sumner CJ (2009): Emerging treatment options for spinal muscular atrophy. Curr Treat Options Neurol 11, 90-101

Byers RK, Banker BQ (1961): Infantile muscular atrophy. Arch Neurol $\underline{5}, 140-164$

Campbell RM, Smith MD (2007): Thoracic insufficiency syndrome and exotic scoliosis. J

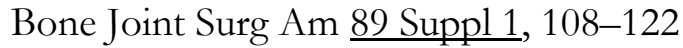

Campbell RM, Smith MD, Mayes TC, Mangos JA, Willey-Courand DB, Kose N, Pinero RF, Alder ME, Duong HL, Surber JL (2003): The characteristics of thoracic insufficiency syndrome associated with fused ribs and congenital scoliosis. J Bone Joint Surg Am ㅎ-A , 399-408

Campbell RM, Smith MD, Mayes TC, Mangos JA, Willey-Courand DB, Kose N, Pinero RF, Alder ME, Duong HL, Surber JL (2004): The effect of opening wedge thoracostomy on thoracic insufficiency syndrome associated with fused ribs and congenital scoliosis. J Bone Joint Surg Am 6-A, $1659-1674$

Canavese F, Sussman MD (2009): Strategies of hip management in neuromuscular disorders: Duchenne Muscular Dystrophy, Spinal Muscular Atrophy, CharcotMarie-Tooth Disease and Arthrogryposis Multiplex Congenita. Hip Int J Clin Exp Res Hip Pathol Ther 19 Suppl 6, 46-52

Canavese F, Rousset M, Le Gledic B, Samba A, Dimeglio A (2014): Surgical advances in the treatment of neuromuscular scoliosis. World J Orthop $\underline{5}, 124-133$

Carstens C (1999): Die neuromuskuläre Skoliose. Der Orthopäde 28, 622-633

Castro D, Iannaccone ST (2014): Spinal Muscular Atrophy: Therapeutic Strategies. Curr Treat Options Neurol $\underline{16}, 316$

Chandran S, McCarthy J, Noonan K, Mann D, Nemeth B, Guiliani T (2011): Early treatment of scoliosis with growing rods in children with severe spinal muscular atrophy: a preliminary report. J Pediatr Orthop $\underline{31}$, 450-454

Chen Z, Li S, Qiu Y, Zhu Z, Chen X, Xu L, Sun X (2017a): Evolution of the postoperative sagittal spinal profile in early-onset scoliosis: is there a difference between rib-based and spine-based growth-friendly instrumentation? J Neurosurg Pediatr 20, 561-566

Chen Z, Qiu Y, Zhu Z, Li S, Chen X, Sun X (2017b): How does hyperkyphotic early-onset scoliosis respond to growing rod treatment? J Pediatr Orthop $\underline{37}$, e593-e598

Cheung JPY, Yiu K, Kwan K, Cheung KMC (2019): Mean 6-year follow-up of magnetically controlled growing rod patients with early onset scoliosis: A glimpse of what happens to graduates. Neurosurgery $\underline{84}, 1112-1123$

Cheung KM-C, Cheung JP-Y, Samartzis D, Mak K-C, Wong Y-W, Cheung W-Y, Akbarnia BA, Luk KD-K (2012): Magnetically controlled growing rods for severe spinal 
curvature in young children: a prospective case series. Lancet Lond Engl $\underline{379}$, 1967-1974

Chua K, Tan CY, Chen Z, Wong HK, Lee EH, Tay SKH, Ong HT, Goh DYT, Hui JHP (2016): Long-term follow-up of pulmonary function and scoliosis in patients with duchenne's muscular dystrophy and spinal muscular atrophy. J Pediatr Orthop $\underline{36}$, 63-69

Cobb WM (1948): Precision in anthropometric techniques; the measurement of stature. Am J Phys Anthropol $\underline{6}, 234$

Cottrill E, Margalit A, Brucker C, Sponseller PD (2019): Comparison of sacral-alar-iliac and iliac-only methods of pelvic fixation in early-onset scoliosis at 5.8 years' mean follow-up. Spine Deform 7, 364-370

Evans GA, Drennan JC, Russman BS (1981): Functional classification and orthopaedic management of spinal muscular atrophy. J Bone Joint Surg Br $\underline{63 B}$, 516-522

Finkel RS, Mercuri E, Darras BT, Connolly AM, Kuntz NL, Kirschner J, Chiriboga CA, Saito K, Servais L, Tizzano E, Topaloglu H, Tulinius M et al. (2017): Nusinersen versus sham control in infantile-onset spinal muscular atrophy. N Engl J Med $\underline{377}$, $1723-1723$

Fletcher ND, Bruce RW (2012): Early onset scoliosis: current concepts and controversies. Curr Rev Musculoskelet Med $\underline{5}, 102-110$

Flynn JM, Tomlinson LA, Pawelek J, Thompson GH, McCarthy R, Akbarnia BA, Growing Spine Study Group (2013): Growing-rod graduates: lessons learned from ninetynine patients who completed lengthening. J Bone Joint Surg Am 모, 1745-1750

Fujak A, Raab W, Schuh A, Kreß A, Forst R, Forst J (2012): Operative treatment of scoliosis in proximal spinal muscular atrophy: results of 41 patients. Arch Orthop Trauma Surg 132, 1697-1706

Fujak A, Raab W, Schuh A, Richter S, Forst R, Forst J (2013): Natural course of scoliosis in proximal spinal muscular atrophy type II and IIIa: descriptive clinical study with retrospective data collection of 126 patients. BMC Musculoskelet Disord $\underline{14}, 283$

Giglio CA, Volpon JB (2007): Development and evaluation of thoracic kyphosis and lumbar lordosis during growth. J Child Orthop 1, 187-193

Gillingham BL, Fan RA, Akbarnia BA (2006): Early onset idiopathic scoliosis. J Am Acad Orthop Surg 14, 101-112

Granata C, Merlini L, Magni E, Marini ML, Stagni SB (1989): Spinal muscular atrophy: natural history and orthopaedic treatment of scoliosis. Spine $\underline{14}, 760-762$

Granata C, Magni E, Merlini L, Cervellati S (1990): Hip dislocation in spinal muscular atrophy. Chir Organi Mov $\underline{75}, 177-184$

Groenefeld B, Hell AK (2013): Ossifications after vertical expandable prosthetic titanium rib treatment in children with thoracic insufficiency syndrome and scoliosis. Spine $\underline{38}, \mathrm{E} 819-823$ 
Groenefeld K: Skoliose-Therapie bei Kindern und Jugendlichen mit dem MAGEC ${ }^{\circledR}$ (magnetic expansion control)-Spinalsystem in Kombination mit dem VEPTR (vertical expandable prosthetic titanium rib)-Instrumentarium. Med. Diss. Göttingen 2017

Haaker G, Fujak A (2013): Proximal spinal muscular atrophy: current orthopedic perspective. Appl Clin Genet $\underline{6}, 113-120$

Hasler C-C, Mehrkens A, Hefti F (2010): Efficacy and safety of VEPTR instrumentation for progressive spine deformities in young children without rib fusions. Eur Spine J $\underline{19}, 400-408$

Hell AK, Campbell RM, Hefti F (2005): The vertical expandable prosthetic titanium rib implant for the treatment of thoracic insufficiency syndrome associated with congenital and neuromuscular scoliosis in young children. J Pediatr Orthop Part B $\underline{14}, 287-293$

Hell AK, Groenefeld K, Tsaknakis K, Braunschweig L, Lorenz HM (2018): Combining bilateral magnetically controlled implants inserted parallel to the spine with rib to pelvis fixation: surgical technique and early results. Clin Spine Surg 31, 239-246

Hell AK, Braunschweig L, Tsaknakis K, von Deimling U, Lüders KA, Hecker M, Lorenz HM (2020): Children with spinal muscular atrophy with prior growth-friendly spinal implants have better results after definite spinal fusion in comparison to untreated patients. Neurosurgery, (im Druck)

Holt JB, Dolan LA, Weinstein SL (2017): Outcomes of primary posterior spinal fusion for scoliosis in spinal muscular atrophy: Clinical, radiographic, and pulmonary outcomes and complications. J Pediatr Orthop $\underline{37}$, e505-e511

Hosseini P, Akbarnia BA (2019): Commentary: Mean 6-year follow-up of magnetically controlled growing rod patients with early onset scoliosis: A glimpse of what happens to graduates. Neurosurgery $\underline{84}$, E267-E268

Hosseini P, Pawelek J, Mundis GM, Yaszay B, Ferguson J, Helenius I, Cheung KM, Demirkiran G, Alanay A, Senkoylu A et al. (2016): Magnetically controlled growing rods for early-onset scoliosis: a multicenter study of 23 cases with minimum 2 years follow-up. Spine $\underline{41}, 1456-1462$

Islander G (2013): Anesthesia and spinal muscle atrophy. Paediatr Anaesth 23, 804-816

Keskinen H, Helenius I, Nnadi C, Cheung K, Ferguson J, Mundis G, Pawelek J, Akbarnia BA (2016): Preliminary comparison of primary and conversion surgery with magnetically controlled growing rods in children with early onset scoliosis. Eur Spine J 25, 3294-3300

Kocyigit IA, Olgun ZD, Demirkiran HG, Ayvaz M, Yazici M (2017): Graduation protocol after growing-rod treatment: removal of implants without new instrumentation is not a realistic approach. J Bone Joint Surg Am 99, 1554-1564

Kolb SJ, Kissel JT (2011): Spinal muscular atrophy: a timely review. Arch Neurol 68, 979_ 984 
Kromeyer-Hauschild K, Wabitsch M, Kunze D, Geller F, Geiß H, Hesse V, von Hippel A, Jaeger U, Johnsen D, Korte W (2001): Perzentile für den Body-mass-Index für das Kindes- und Jugendalter unter Heranziehung verschiedener deutscher Stichproben. Monatsschr Kinderheilkd 149,807-818

Lampe LP, Bövingloh AS, Gosheger G, Schulte TL, Lange T (2019): Magnetically controlled growing rods in treatment of early-onset scoliosis: a single center study with a minimum of 2-year-follow up and preliminary results after converting Surgery. Spine 44(17), 1201-1210

Lefebvre S, Bürglen L, Reboullet S, Clermont O, Burlet P, Viollet L, Benichou B, Cruaud C, Millasseau P, Zeviani M (1995): Identification and characterization of a spinal muscular atrophy-determining gene. Cell $\underline{80}, 155-165$

Lefebvre S, Burlet P, Liu Q, Bertrandy S, Clermont O, Munnich A, Dreyfuss G, Melki J (1997): Correlation between severity and SMN protein level in spinal muscular atrophy. Nat Genet 16, 265-269

Lorenz HM, Badwan B, Hecker MM, Tsaknakis K, Groenefeld K, Braunschweig L, Hell AK (2017): Magnetically controlled devices parallel to the spine in children with spinal muscular atrophy. JB JS Open Access $\underline{2}$, e0036

Lorenz HM, Kühnle I, Edler J, Hobbiebrunken E, Wilichowski E, Tsaknakis K, Wilken B, Braunschweig L, Hell AK (2018): Intrathekale Nusinersen-Therapie bei Kindern mit Spinaler Muskelatrophie und Wirbelsäulendeformitäten. Klin Padiatr 230, 231233

Lorenz HM, Braunschweig L, Badwan B, Groenefeld K, Hecker MM, Tsaknakis K, Grote J, Hell AK (2019): High correlation between achieved and expected distraction using magnetically controlled growth rods (MCGR) with rib to pelvis fixation in pediatric spine deformity. J Pediatr Orthop $\underline{39}$, e334-e338

Lunn MR, Wang CH (2008): Spinal muscular atrophy. The Lancet $\underline{371}$, 2120-2133

Markowitz JA, Singh P, Darras BT (2012): Spinal muscular atrophy: A clinical and research update. Pediatr Neurol $\underline{46}, 1-12$

McElroy MJ, Shaner AC, Crawford TO, Thompson GH, Kadakia RV, Akbarnia BA, Skaggs DL, Emans JB, Sponseller PD (2011): Growing rods for scoliosis in spinal muscular atrophy: structural effects, complications, and hospital stays. Spine $\underline{36}$, 1305-1311

Mercuri E, Bertini E, Iannaccone ST (2012): Childhood spinal muscular atrophy: controversies and challenges. Lancet Neurol 11, 443-452

Mercuri E, Darras BT, Chiriboga CA, Day JW, Campbell C, Connolly AM, Iannaccone ST, Kirschner J, Kuntz NL, Saito K, et al. (2018): Nusinersen versus sham control in later-onset spinal muscular atrophy. N Engl J Med $\underline{378}$, 625-635

Patel J, Shapiro F (2015): Simultaneous progression patterns of scoliosis, pelvic obliquity, and hip subluxation/dislocation in non-ambulatory neuromuscular patients: an approach to deformity documentation. J Child Orthop 2 , 345-356 
Pechmann A, König K, Bernert G, Schachtrup K, Schara U, Schorling D, Schwersenz I, Stein S, Tassoni A, Vogt S, et al. (2019): SMArtCARE - A platform to collect reallife outcome data of patients with spinal muscular atrophy. Orphanet J Rare Dis $\underline{14}$, 18

Pizones J, Martín-Buitrago MP, Sánchez Márquez JM, Fernández-Baíllo N, Baldan-Martin M, Sánchez Pérez-Grueso FJ (2018): Decision making of graduation in patients with early-onset scoliosis at the end of distraction-based programs: risks and benefits of definitive fusion. Spine Deform $\underline{6}, 308-313$

Plaass C, Hasler CC, Heininger U, Studer D (2016): Bacterial colonization of VEPTR implants under repeated expansions in children with severe early onset spinal deformities. Eur Spine J 25, 549-556

Putzier M, Groß C, Zahn RK, Pumberger M, Strube P (2016): Besonderheiten neuromuskulärer Skoliosen. Orthopäde 4도 , 500-508

Ridderbusch K, Rupprecht M, Kunkel P, Hagemann C, Stücker R (2017): Preliminary results of magnetically controlled growing rods for early onset scoliosis. J Pediatr Orthop $\underline{37}$, e575-e580

Ridderbusch K, Spiro AS, Kunkel P, Grolle B, Stücker R, Rupprecht M (2018): Strategies for treating scoliosis in early childhood. Dtsch Arzteblatt Int 115, 371-376

Rumalla K, Yarbrough CK, Pugely AJ, Koester L, Dorward IG (2016): Spinal fusion for pediatric neuromuscular scoliosis: national trends, complications, and in-hospital outcomes. J Neurosurg Spine $\underline{25}, 500-508$

Russman BS (2007): Spinal muscular atrophy: clinical classification and disease heterogeneity. J Child Neurol 22, 946-951

Russman BS, Iannacone ST, Buncher CR, Samaha FJ, White M, Perkins B, Zimmerman L, Smith C, Burhans K, Barker L (1992): Spinal muscular atrophy: new thoughts on the pathogenesis and classification schema. J Child Neurol 7, 347-353

Sankar WN, Skaggs DL, Yazici M, Johnston CE, Shah SA, Javidan P, Kadakia RV, Day TF, Akbarnia BA (2011): Lengthening of dual growing rods and the law of diminishing returns. Spine $\underline{36}, 806-809$

Scholten PJ, Veldhuizen AG (1987): Analysis of Cobb angle measurements in scoliosis. Clin Biomech Bristol Avon 2 , 7-13

Schwentker EP, Gibson DA (1976): The orthopaedic aspects of spinal muscular atrophy. J Bone Joint Surg Am 모, 32-38

Shorrock HK, Gillingwater TH, Groen EJN (2018): Overview of current drugs and molecules in development for spinal muscular atrophy therapy. Drugs $\underline{78}, 293-305$

Sporer SM, Smith BG (2003): Hip dislocation in patients with spinal muscular atrophy. J Pediatr Orthop $\underline{23}, 10-14$

Strauss KA, Carson VJ, Brigatti KW, Young M, Robinson DL, Hendrickson C, Fox MD, Reed RM, Puffenberger EG, Mackenzie W, Miller F (2018): Preliminary safety and tolerability of a novel subcutaneous intrathecal catheter system for repeated 
outpatient dosing of nusinersen to children and adults with spinal muscular atrophy. J Pediatr Orthop $\underline{38}$, e610-e617

Studer D, Heidt C, Büchler P, Hasler CC (2019): Treatment of early onset spinal deformities with magnetically controlled growing rods: a single centre experience of 30 cases. J Child Orthop 13, 196-205

Tang N, Zhao H, Shen J X, Zhang J G, Li S G (2019): Magnetically controlled growing rod for early-onset scoliosis: systematic review and meta-analysis. World Neurosurg $\underline{125}$, e593-e601

Thakar C, Kieser DC, Mardare M, Haleem S, Fairbank J, Nnadi C (2018): Systematic review of the complications associated with magnetically controlled growing rods for the treatment of early onset scoliosis. Eur Spine J 27, 2062-2071

Thompson W, Thakar C, Rolton DJ, Wilson-MacDonald J, Nnadi C (2016): The use of magnetically-controlled growing rods to treat children with early-onset scoliosis: early radiological results in 19 children. Bone Jt J $\underline{98-B}, 1240-1247$

Tis JE, Karlin LI, Akbarnia BA, Blakemore LC, Thompson GH, McCarthy RE, Tello CA, Mendelow MJ, Southern EP, Growing Spine Committee of the Scoliosis Research Society (2012): Early onset scoliosis: modern treatment and results. J Pediatr Orthop $\underline{32}$, 647-657

Trobisch P, Suess O, Schwab F (2010): Idiopathic Scoliosis. Dtsch Ärztebl Int 107, 875884

Wagner L, Braunschweig L, Eiffert H, Tsaknakis K, Kamin D, D’Este E, Messer PK, Hell AK, Lorenz HM (2018): Detection of bacteria colonizing titanium spinal implants in children. Surg Infect 19, 71-77

Waldrop MA, Kolb SJ (2019): Current treatment options in neurology -SMA therapeutics. Curr Treat Options Neurol 21, 25

Watts SL (2016): Use of a vertical expandable prosthetic titanium rib in children with thoracic insufficiency syndrome and scoliosis. Crit Care Nurse $\underline{36}, 52-61$

Wu A-M, Cheung JPY, Cheung KMC, Lin J L, Jin H M, Chen D, Wang X Y, Zhao J, Kwan KYH (2019): Minimum 2-year experience with magnetically controlled growing rods for the treatment of early-onset scoliosis: a systematic review. Asian Spine J 13(4), 682-693

Yang S, Andras LM, Redding GJ, Skaggs DL (2016): Early-onset scoliosis: a review of history, current treatment, and future directions. Pediatrics $\underline{137}$, Issue 1

Zebala LP, Bridwell KH, Baldus C, Richards SB, Dormans JP, Lenke LG, Auerbach JD, Lovejoy J (2011): Minimum 5-year radiographic results of long scoliosis fusion in juvenile spinal muscular atrophy patients: major curve progression after instrumented fusion. J Pediatr Orthop $\underline{31}$, 480-488

Zenios M, Sampath J, Cole C, Khan T, Galasko CSB (2005): Operative treatment for hip

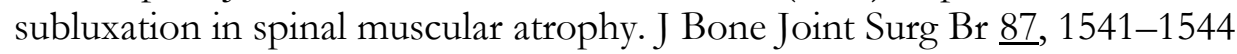


Zivkovic V, Büchler P, Ovadia D, Riise R, Stuecker R, Hasler C (2014): Extraspinal ossifications after implantation of vertical expandable prosthetic titanium ribs (VEPTRs). J Child Orthop $\underline{8}, 237-244$ 


\section{Danksagung}

Vielen lieben Dank richte ich an die komplette Arbeitsgruppe der Kinderorthopädie der Universitätsmedizin Göttingen, die ein wirklich großartiges Team darstellt. Es ist beeindruckend, wie sie den Arbeitsaufwand des Klinikalltages und zusätzlich die wissenschaftliche Arbeit so professionell bewältigt. Es ist von unschätzbarem Wert, wie sie mir einen Teil ihrer kostbaren Zeit geschenkt hat, um mich durch die Promotion zu begleiten.

Ganz herzlich danke ich meiner Doktormutter Frau Prof. Dr. med. Anna K. Hell für die Möglichkeit eine Promotion im Bereich der Kinderorthopädie absolvieren zu können, was mein Wunsch gewesen ist. Sie ist eine außergewöhnlich gute Chefin und Lehrerin, die gleichzeitig aufmunternde und bestärkende Worte findet und auch mit hoher Erwartungshaltung Leistung einfordert. Für mich war das eine gute Kombination, die motiviert hat Einsatz zu zeigen, sich stetig zu bemühen und sich immer verbessern zu wollen. Außerdem möchte ich für jede Chance und jede Tür danken, die sich für mich ganz unerwartet geöffnet hat. Dazu gehören Mitautorenschaften von Veröffentlichungen genauso wie die Unterstützung für das Studentenstipendium der Jahrestagung der Vereinigung für Kinderorthopädie 2019.

Ferner danke ich dem Betreuer meiner Doktorarbeit Herrn Dr. med. Heiko Lorenz. Nicht nur als Betreuer, sondern auch als Arzt oder Forscher, ist Dr. Lorenz immer mit vollem Einsatz dabei und arbeitet leidenschaftlich und mit perfektionistischer Liebe zum Detail. Vielen Dank für den besonderen Einsatz für dieses Projekt und für all die Stunden der Freizeit, die er der Programmierung der Datenbank geopfert hat. Sie ist über die Zeit mitgewachsen und hat inzwischen so viele großartige Funktionen. Sehr dankbar bin ich auch dafür, dass ich immer einen herzensguten Freund an meiner Seite wusste, mit dem das gemeinsame Arbeiten viel Spaß gemacht hat, und einen liebevollen Helfer, der zu jeder Zeit für mich da war.

Nicht zuletzt gilt mein Dank Frau Dr. Lena Braunschweig. Sie war die gute Seele, die immer in ihrem Büro anzutreffen war und ganz kurz Zeit für einen Tee und einen Plausch zu dem neusten Stand der Arbeit hatte. Mein Dank gilt ihrem offenen Ohr bei Fragen und ihrer helfenden Hand zum Beispiel bei der Erstellung der Graphen. 